\title{
LARGE EXCURSIONS AND CONDITIONED LAWS FOR RECURSIVE SEQUENCES GENERATED BY RANDOM MATRICES
}

\author{
By JefFrey F. Collamore and Sebastian Mentemeier \\ University of Copenhagen and TU Dortmund
}

We study the large exceedance probabilities and large exceedance paths of the recursive sequence $V_{n}=M_{n} V_{n-1}+Q_{n}$, where $\left\{\left(M_{n}, Q_{n}\right)\right\}$ is an i.i.d. sequence, and $M_{1}$ is a $d \times d$ random matrix and $Q_{1}$ is a random vector, both with nonnegative entries. We impose conditions which guarantee the existence of a unique stationary distribution for $\left\{V_{n}\right\}$ and a Cramér-type condition for $\left\{M_{n}\right\}$. Under these assumptions, we characterize the distribution of the first passage time $T_{u}^{A}:=\inf \left\{n: V_{n} \in u A\right\}$, where $A$ is a general subset of $\mathbb{R}^{d}$, exhibiting that $T_{u}^{A} / u^{\alpha}$ converges to an exponential law for a certain $\alpha>0$. In the process, we revisit and refine classical estimates for $\mathbb{P}(V \in u A)$, where $V$ possesses the stationary law of $\left\{V_{n}\right\}$. Namely, for $A \subset \mathbb{R}^{d}$, we show that $\mathbb{P}(V \in u A) \sim C_{A} u^{-\alpha}$ as $u \rightarrow \infty$, providing, most importantly, a new characterization of the constant $C_{A}$. As a simple consequence of these estimates, we also obtain an expression for the extremal index of $\left\{\left|V_{n}\right|\right\}$. Finally, we describe the large exceedance paths via two conditioned limit theorems showing, roughly, that $\left\{V_{n}\right\}$ follows an exponentially-shifted Markov random walk, which we identify. We thereby generalize results from the theory of classical random walk to multivariate recursive sequences.

\section{CONTENTS}

1. Introduction . . . . . . . . . . . . . . . . . . . . . . . . 2065

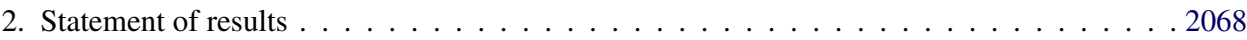

2.1. Notation . . . . . . . . . . . . . . . . . . . . . . . . . 2068

2.2. Basic assumptions . . . . . . . . . . . . . . . . . . . . . . . . . . . . . 2069

Allowable and positively regular matrices . . . . . . . . . . . . . . . . . . . . . 2069

Nonarithmetic distributions for random matrices . . . . . . . . . . . . . . . . . . . 2069

The shifted distribution . . . . . . . . . . . . . . . . . . . . . . . . 2071

The Markov random walk . . . . . . . . . . . . . . . . . . . . . . 2072

Probability measures . . . . . . . . . . . . . . . . . . . . . . . . . . . 2072

2.3. Tail estimates for $\left\{V_{n}\right\} \quad \ldots \ldots \ldots 2072$

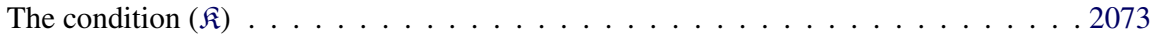

2.4. Extremal estimates for maxima and first passage times . . . . . . . . . . . . . . . 2075

2.5. The path and empirical law under a large exceedance . . . . . . . . . . . . . . 2077

3. Background . . . . . . . . . . . . . . . . . . . . . . . . . 2078

3.1. Preliminary results from Markov chain theory . . . . . . . . . . . . . . 2078

Received August 2016; revised July 2017.

MSC2010 subject classifications. Primary 60K15, 60F10; secondary 60J05, 60G70, 60G17.

Key words and phrases. Random recurrence equations, stochastic fixed-point equations, products of random matrices, Markov chain theory in general state space, nonlinear Markov renewal theory, large deviations, first passage times, conditional limit theorems, extreme value theory. 
3.2. Quantifying the discrepancy between $\left\{V_{n}\right\}$ and $\left\{e^{S_{n}} X_{n}\right\} \ldots \ldots \ldots 2081$

3.3. Markov nonlinear renewal theory . . . . . . . . . . . . . . . . . . . . . . 2086

4. Characterizing the large exceedances over cycles _ . . . . . . . . . . . . . . . . . 2090

4.1. Proposition 4.1 and its consequences . . . . . . . . . . . . . . . . . . . . . 2090

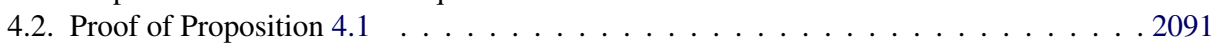

4.3. Toward the proof of Theorem $2.4 \ldots \ldots \ldots$

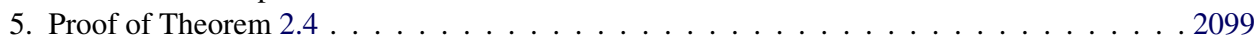

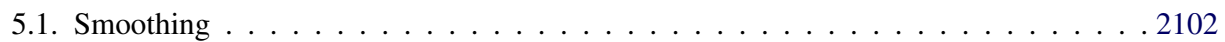

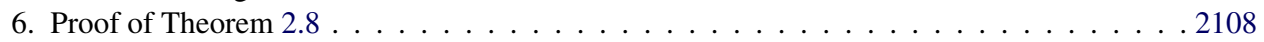

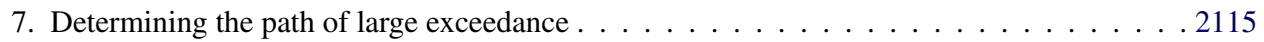

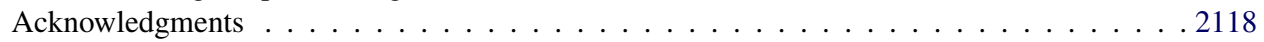

References . . . . . . . . . . . . . . . . . . . . . . 2118

1. Introduction. The goal of this paper is to describe the extremal behavior and tail asymptotics, and to develop certain conditioned limit theorems, for the multivariate recursive sequence

$$
V_{n}=M_{n} V_{n-1}+Q_{n}, \quad n=1,2, \ldots, \quad V_{0} \sim \gamma,
$$

where $\left\{\left(M_{n}, Q_{n}\right)\right\}$ is an i.i.d. sequence, $M_{1}$ is a $d \times d$ random matrix with nonnegative entries, and is $Q_{1}$ a nonnegative random vector, and the initial measure $\gamma$ in (1.1) is supported on the nonnegative orthant and independent of $\left\{\left(M_{n}, Q_{n}\right)\right\}$ (typically taken to be point mass at $v \in[0, \infty)^{d}$ ). We allow for an arbitrary dependence structure between $M_{1}$ and $Q_{1}$.

Motivated by branching processes in random environments with immigration, as considered by Solomon [47, 48], the recursive sequence (1.1) was originally studied in the fundamental paper of Kesten [28]. Assuming that the top Lyapunov exponent for $\left\{M_{n}\right\}$ is negative, then the Markov chain $\left\{V_{n}\right\}$ has a unique stationary distribution; and if $V$ is a random variable possessing the stationary law of $\left\{V_{n}\right\}$, then it is shown in [28] that under appropriate moment and irreducibility conditions,

$$
\mathbb{P}(\langle w, V\rangle>u) \sim \mathscr{C}_{w} u^{-\alpha} \quad \text { as } u \rightarrow \infty,
$$

for any vector $w \in(0, \infty)^{d}$ and some constant $\mathscr{C}_{w}>0$.

Recently, there has been a renewed interest in Kesten's estimate. For example, the asymptotics in (1.2) have been shown to characterize the stationary tail decay in the $\operatorname{GARCH}(p, q)$ financial time series model or, analogously, the $\operatorname{ARMA}(p, q)$ process with random coefficients; $\mathrm{cf}$. $[19,38]$. The process $(1.1)$ is also relevant for the study of random walk in random environment (cf., e.g., [30, 50]), and in a variety of other problems related to branching processes and Mandelbrot cascades; cf. [11, 24, 33] and references therein. Furthermore, in recent years, the scope of Kesten's method has broadened to include more general fixed-point equations in $\mathbb{R}$; namely equations of the form $V \stackrel{d}{=} F(V)$, where $F: \mathbb{R} \rightarrow \mathbb{R}$ is a random function independent of $V$, and $F(v) \approx M v$ for large $v$, where $M$ is a random variable 
in $\mathbb{R}$; cf. [4, 18, 23, 39]. (Here, $\stackrel{d}{=}$ denotes equality in distribution.) Moreover, generalizations to Markov-dependent recursive sequences (satisfying different assumptions from those we consider here) have been obtained in [14, 17, 44].

It is natural to ask whether this theory may be extended to reveal more refined path properties of the process $\left\{V_{n}\right\}$. In fact, some characteristics of $\left\{V_{n}\right\}$ over large excursions can essentially be inferred from those of the Markov random walk $\left\{\left(X_{n}, S_{n}\right): n=0,1, \ldots\right\}$, defined by

$$
X_{n}=\frac{M_{n} \cdots M_{1} X_{0}}{\left|M_{n} \cdots M_{1} X_{0}\right|}, \quad S_{n}=\log \left|M_{n} \cdots M_{1} X_{0}\right|,
$$

where $|\cdot|$ denotes a norm in $\mathbb{R}^{d}$, and $X_{0}$ can be taken to be the projection of $V_{0}$ onto the unit sphere. While the rough equivalence between $\left\{V_{n}\right\}$ and $\left\{e^{S_{n}} X_{n}\right\}$ has been utilized by numerous authors, including Kesten [28], the correspondence between these processes has typically only been employed to obtain estimates such as (1.2), and not to characterize more detailed path properties. In contrast, our approach will be to quantify this discrepancy using Markov nonlinear renewal theory, as developed in Melfi [34, 35], yielding-after accounting for the small-time behavior-estimates which show that $\left\{V_{n}\right\}$ is closely approximated by $\left\{e^{S_{n}} X_{n}\right\}$ in a manner which we characterize mathematically. Consequently, it is natural to expect that, over a large excursion, the random walk structure inherent in $\left\{\left(X_{n}, S_{n}\right)\right\}$ may be exploited to yield deeper properties of $\left\{V_{n}\right\}$ which mimic known attributes of Markov random walk. Following this approach, we shall reexamine Kesten's estimate, then extend the approach to obtain related asymptotic results relevant in extreme value theory, and, ultimately, derive certain path estimates conditioned on a large excursion, showing quantitatively that the path of $\left\{V_{n}\right\}$ under a large excursion resembles a Markov random walk, but in an exponentially-tilted measure (which we will identify as the " $\alpha$-shifted measure" below).

We start by revisiting (1.2), establishing under appropriate conditions that, for any set $A \subset[0, \infty)^{d}$ with positive distance to the origin,

$$
\mathbb{P}(V \in u A) \sim \frac{C}{\lambda^{\prime}(\alpha)} \mathfrak{L}_{\alpha}(A) u^{-\alpha} \quad \text { as } u \rightarrow \infty,
$$

for a universal constant $C$ and a measure $\mathfrak{L}_{\alpha}$. In particular, we obtain a new representation of the constant $C$ as the $\alpha$ th moment of a certain power series derived from $\left\{\left(M_{n}, Q_{n}\right)\right\}$ and the time-reversed products of $\left\{M_{n}\right\}$; see (2.9) and (2.10) below. The formula we derive can be viewed as a multidimensional extension of a central result in [18]. (For related one-dimensional estimates, see also [12,21] and the discussion in Section 2.3 below.) From (1.4), we immediately conclude that $V$ is multivariate regularly varying, as could only be deduced from (1.2) with the help of the Cramér-Wold device; cf. [6,8]. We emphasize that this additional step is not needed in our method. 
Following a similar approach, we then examine the extremal behavior of $\left\{V_{n}\right\}$. Specifically, letting $A \subset(0, \infty)^{d}$ have a positive distance to the origin and setting $T_{u}^{A}=\inf \left\{n: V_{n} \in u A\right\}$, we study the growth rate of $T_{u}^{A}$ as $u \rightarrow \infty$. We show that

$$
\lim _{u \rightarrow \infty} \mathbb{P}\left(\frac{T_{u}^{A}}{u^{\alpha}} \leq z \mid V_{0}=v\right)=e^{-K_{A} z}, \quad z \geq 0,
$$

where $\alpha$ is given as in (1.2) and $K_{A}$ is a constant which we also characterize, relating this constant explicitly to $C$ and to the pre-factor appearing in the asymptotic expression, as $u \rightarrow \infty$, for the hitting probability of the set $u A$ by $\left\{e^{S_{n}} X_{n}\right\}$. As a special case, setting $A=\{x:|x|>1\}$, we then conclude that $\left\{\left|V_{n}\right|\right\}$ belongs to the maximum domain of attraction of the Fréchet distribution. However, it should be emphasized that (1.5) is actually a stronger result, yielding the directional dependence of $\left\{V_{n}\right\}$ and suggesting a natural extension of classical extreme value theory to this multidimensional setting. Note that (1.5) characterizes the first passage times of the "forward" iterates $\left\{V_{n}\right\}$ (in the sense of Letac [32]), which are qualitatively different from the "backward" iterates. In one dimension, the backward iterates are perpetuities, and the first passage times of these sequences have recently been studied in [9], yielding very different results from those we obtain here. In contrast, (1.5) is qualitatively similar to reflected random walk, and (1.5) can be viewed as an extension, to our setting, of a classical result due to Iglehart [27] and some of its extensions, for example, [20]. In particular, (1.5) sharpens earlier work, largely restricted to one-dimensional recursions, in [11, 19, 41, 42]; cf. Remark 2.9 below.

The key to establishing (1.4) and (1.5) is a proposition, where we study the behavior of $\left\{V_{n}\right\}$ over cycles emanating from, and then returning to, a given set $\mathbb{D} \subset[0, \infty)^{d}$. Drawing an analogy with reflected random walk, these returns to $\mathbb{D}$ play the role of Iglehart's [27] returns of a reflected random walk to the origin. Letting $\tau$ denote the first return time to $\mathbb{D}$, then for any suitable function $g$ and any $m \in\{1,2, \ldots\}$, we consider in Proposition 4.1 the limit behavior of

$$
u^{\alpha} \mathbb{E}\left[g\left(\frac{V_{T_{u}^{A}}}{u}, \ldots, \frac{V_{T_{u}^{A}+m}}{u}\right) \mathbf{1}_{\left\{T_{u}^{A}<\tau\right\}} \mid V_{0}=v\right] \quad \text { as } u \rightarrow \infty .
$$

If $g=1$, then this quantity represents the rescaled probability that $\left\{V_{n}\right\}$ enters the set $u A$ before returning to $\mathbb{D}$. Moreover, for general $g$, we show that the post- $T_{u}^{A}$ process behaves as $\left\{e^{S_{n}} X_{n}\right\}$, but starting with the stationary overjump distribution. This idea is then extended in the final section of the article to include the path behavior prior to time $T_{u}^{A}$, drawing a close analogy to the trajectory of $\left\{e^{S_{n}} X_{n}\right\}$ in the $\alpha$-shifted measure.

Namely, we develop two conditioned limit theorems. In the first, we study the empirical law of $\left\{\log \left|V_{n}\right|-\log \left|V_{n-1}\right|\right\}$ conditioned on $\left\{T_{u}^{A}<\tau\right\}$, showing that this empirical law converges weakly in $\mathbb{P}\left(\cdot \mid T_{u}^{A}<\tau\right)$-probability to the distribution, 
under stationarity, of $S_{1}$ in the $\alpha$-shifted measure. We also establish a result concerning the joint distribution of $\left\{V_{I_{u}}, V_{I_{u}+1}, \ldots\right\}$ conditioned on $\left\{T_{u}^{A}<\tau\right\}$, where $I_{u}$ grows "slowly" compared with $u$.

We emphasize that we shall develop our limit theorems without assuming that the process $\left\{V_{n}\right\}$ is Harris recurrent, and thus-while we shall often draw upon the theory of Harris recurrent chains and these methods will play an important role in our analysis - our approach will ultimately not require this standard assumption from Markov chain theory, which is unnatural in our setting. We circumvent this requirement by introducing a smoothing technique, where the sequence $\left\{Q_{k n}\right\}$ is "smoothed" for some $k \in\{1,2, \ldots\}$, thereby ensuring that the resulting process is Harris recurrent, yet the effect of this smoothing is negligible in an asymptotic limit. This technique could also be adapted to other recursive sequences satisfying a stochastic fixed-point equation (as considered in the one-dimensional setting in [18]). To obtain a general theory without Harris recurrence, we shall, instead, rely throughout the article on the recently-developed theory of Guivarc'h and Le Page [25], which exploits spectral gap properties on special function spaces for matrix products under weak regularity conditions. While the theory in [25] is developed for invertible matrices, a formulation for matrices with nonnegative entries, as we shall consider here, has recently been given in [10].

We now turn to a precise statement of our main results.

\section{Statement of results.}

2.1. Notation. Let $\mathbb{N}_{+}:=\{1,2, \ldots\}$ denote the positive integers. For given $d \in \mathbb{N}_{+}$, assume that $\mathbb{R}^{d}$ is endowed with the scalar product $\langle\cdot, \cdot\rangle$ and canonical orthonormal basis $\left\{e_{i}\right\}$. Set $\mathbb{R}_{+}^{d}=\left\{x \in \mathbb{R}^{d}:\left\langle x, e_{i}\right\rangle \geq 0,1 \leq i \leq d\right\}$.

Let $|\cdot|$ denote a norm in $\mathbb{R}_{+}^{d}$, and assume throughout the article that $|\cdot|$ is monotone, that is, if $x, y \in \mathbb{R}_{+}^{d}$ satisfy $y-x \in \mathbb{R}_{+}^{d}$, then $|x| \leq|y|$. Let $\mathbb{S}^{d-1}:=$ $\left\{x \in \mathbb{R}^{d}:|x|=1\right\}$ denote the unit sphere and $\mathbb{S}_{+}^{d-1}:=\mathbb{R}_{+}^{d} \cap \mathbb{S}^{d-1}$; and for any $x \in \mathbb{R}^{d} \backslash\{0\}$, let $\tilde{x}$ denote its projection onto the unit sphere, namely

$$
\tilde{x} \equiv(x)^{\sim}:=|x|^{-1} x .
$$

Set $B_{r}(y)=\left\{x \in \mathbb{R}^{d}:|x-y|<r\right\}, r>0$; and $B_{r}^{+}(y)=B_{r}(y) \cap \mathbb{R}_{+}^{d}$.

For any subspace $\mathscr{S}$ of $\mathbb{R}_{+}^{d}$, let $\mathscr{B}(\mathscr{S})$ denote the collection of Borel sets on $\mathscr{S}$; and let $E^{\circ}, \bar{E}, E^{c}$, and $\partial E$ denote the interior, closure, complement and boundary of $E \in \mathscr{B}(\mathscr{S})$, respectively. For any measure $v$ on $\mathscr{S} \subset \mathbb{R}_{+}^{d}$, denote the support of $v$ by supp $v$. Also, denote the set of bounded continuous real-valued functions on a space $E$ by $\mathscr{C}_{b}(E)$, equipped with the norm $|f|_{\infty}:=\sup \{|f(x)|: x \in E\}$.

Let $\mathfrak{M}$ denote the collection of $d \times d$ matrices with nonnegative coefficients, and let $\|\mathfrak{m}\|$ denote operator norm, that is, $\|\mathfrak{m}\|:=\sup _{x \in \mathbb{S}^{d-1}}|\mathfrak{m} x|, \mathfrak{m} \in \mathfrak{M}$.

Now suppose that $\left\{V_{n}\right\}$ and $\left\{\left(M_{n}, Q_{n}\right): n \in \mathbb{N}_{+}\right\}$are defined as in the previous section; in particular, each $\left(M_{n}, Q_{n}\right)$ is an i.i.d. copy of $(M, Q)$, where the random 
matrix $M$ takes values in $\mathfrak{M}$ a.s., $Q$ takes values in $\mathbb{R}_{+}^{d}$ a.s., and we allow the pair $(M, Q)$ to have an arbitrary dependence structure. Denote the probability laws of $(M, Q), M$ and $Q$ by $\mu, \mu_{M}$ and $\mu_{Q}$, respectively. Assume that $\left\{\left(M_{i}, Q_{i}\right): i=\right.$ $1, \ldots, n\}$ is adapted to a given filtration $\left\{\mathscr{F}_{n}: n=1,2, \ldots\right\}$.

\subsection{Basic assumptions. We first introduce certain restrictions on $\left\{M_{n}\right\}$.}

Allowable and positively regular matrices. We say that a matrix $\mathfrak{m} \in \mathfrak{M}$ is allowable if it has no zero row or column. Moreover, if the coefficients of a given matrix $\mathfrak{m} \in \mathfrak{M}$ are strictly positive, then we write $\mathfrak{m} \succ 0$ and say that $\mathfrak{m}$ is positively regular. Also write $\mathfrak{M}^{\circ}=\{\mathfrak{m} \in \mathfrak{M}: \mathfrak{m} \succ 0\}$. As a standing assumption, we shall always assume that there exists an $n \in \mathbb{N}_{+}$such that

$$
\mathfrak{N}:=\inf \left\{n \in \mathbb{N}_{+}: M_{n} \cdots M_{1} \succ 0\right\}<\infty \quad \text { a.s.; }
$$

thus, ultimately, the product $M_{n} \cdots M_{1}$ is positively regular with probability one. This assumption will be subsumed in the stronger Hypothesis $\left(\mathrm{H}_{1}\right)$, given below (cf. [26], Lemma 3.1, or [10], Lemma 6.3).

Nonarithmetic distributions for random matrices. Next, we need a generalization of the notion of a nonarithmetic distribution to the setting of random matrices. To this end, let $\Gamma_{M}$ denote the smallest closed subsemigroup of $\mathfrak{M}$ which contains $\operatorname{supp} \mu_{M}$.

DEFINITION 2.1. We say that $\mu_{M}$ is nonarithmetic if the additive group generated by $\left\{\log \|\mathfrak{m}\|: \mathfrak{m} \in \Gamma_{M} \cap \mathfrak{M}^{\circ}\right\}$ is dense in $\mathbb{R}$.

It is shown in [13], Lemma 2.7, that this condition implies that of Shurenkov [45], which is closer to the condition imposed on one-dimensional Markov random walks, but not easily verified in the setting of random matrices. It is worth observing that, alternatively, we could replace $\log \|\mathfrak{m}\|$ with the Frobenius eigenvalue of $\mathfrak{m}$ in Definition 2.1; thus, our definition is in agreement with the one given by Kesten in [28].

We are now prepared to introduce our basic assumptions on the distribution function $\mu_{M}$ of $M$.

Hypothesis $\left(\mathrm{H}_{1}\right) . \quad \mu_{M}$ is nonarithmetic and $\mu_{M}\{\mathfrak{m}: \mathfrak{m}$ is allowable $\}=1$.

Next, we turn to certain moment conditions that will be imposed on the pair $(M, Q)$. Let

$$
\mathfrak{D}=\left\{\theta \geq 0: \int_{\mathfrak{M}}\|\mathfrak{m}\|^{\theta} \mu_{M}(d \mathfrak{m})<\infty\right\}=\left\{\theta \geq 0: \mathbb{E}\left[\|M\|^{\theta}\right]<\infty\right\}
$$


and let $\mathfrak{m}^{T}$ denote the transpose of $\mathfrak{m}$. Then for any $\theta \in \mathfrak{D}$ and any $f \in \mathscr{C}_{b}\left(\mathbb{S}_{+}^{d-1}\right)$, set

$$
\begin{aligned}
P_{\theta} f(x) & =\mathbb{E}\left[|M x|^{\theta} f(\widetilde{M x})\right] \\
P_{\theta}^{*} f(x) & =\mathbb{E}\left[\left|M^{T} x\right|^{\theta} f\left(\widetilde{M^{T} x}\right)\right] \\
\lambda(\theta) & =\lim _{n \rightarrow \infty}\left(\mathbb{E}\left[\left\|M_{n} \cdots M_{1}\right\|^{\theta}\right]\right)^{1 / n} ; \\
\Lambda(\theta) & =\log \lambda(\theta) .
\end{aligned}
$$

In the following lemma, we describe the left-invariant measures and rightinvariant functions associated with the operators $P_{\theta}$ and $P_{\theta}^{*}$.

LEMMA 2.2. Assume $\theta \in \mathfrak{D}$ and $\mu_{M}\{\mathfrak{m}: \mathfrak{m}$ is allowable $\}=1$. Then $\lambda(\theta)$ is the spectral radius of $P_{\theta}$, and there is a unique probability measure $l_{\theta}$ on $\mathbb{S}_{+}^{d-1}$ and a unique, strictly positive function $r_{\theta} \in \mathscr{C}_{b}\left(\mathbb{S}_{+}^{d-1}\right)$ with $\int r_{\theta}(x) l_{\theta}(d x)=1$ such that

$$
l_{\theta} P_{\theta}=\lambda(\theta) l_{\theta} \quad \text { and } \quad P_{\theta} r_{\theta}=\lambda(\theta) r_{\theta} .
$$

Furthermore, the function $r_{\theta}$ is $\max \{\theta, 1\}$-Hölder continuous; and thus, $r_{\theta}$ is bounded from above and below by finite positive constants.

Similarly, the spectral radius of $P_{\theta}^{*}$ equals $\lambda(\theta)$, and there exists a pair $\left(l_{\theta}^{*}, r_{\theta}^{*}\right)$ which has the equivalent properties, relative to $P_{\theta}^{*}$, as those possessed by $\left(l_{\theta}, r_{\theta}\right)$ relative to $P_{\theta}$. Moreover,

$$
r_{\theta}(x)=c \int_{\mathbb{S}_{+}^{d-1}}\langle x, y\rangle^{\theta} l_{\theta}^{*}(d y), \quad \forall x \in \mathbb{S}_{+}^{d-1},
$$

for $c=\left(\int\langle x, y\rangle^{\alpha} l_{\theta}^{*}(d x) l_{\theta}(d y)\right)^{-1}$. Likewise, (2.2) also holds if $\left(r_{\theta}, l_{\theta}^{*}\right)$ is replaced with $\left(r_{\theta}^{*}, l_{\theta}\right)$.

In the above lemma, we have written $l_{\theta} P_{\theta}$ for the application of the adjoint operator $P_{\theta}^{\prime}$ to the measure $l_{\theta}$; that is, $l_{\theta} P_{\theta}$ is the unique measure satisfying

$$
\int_{\mathbb{S}_{+}^{d-1}} f(x)\left(l_{\theta} P_{\theta}\right)(d x)=\int_{\mathbb{S}_{+}^{d-1}}\left(P_{\theta} f(x)\right) l_{\theta}(d x) \quad \text { for all } f \in \mathscr{C}_{b}\left(\mathbb{S}_{+}^{d-1}\right) .
$$

The proof of Lemma 2.1 can be found in [10], Proposition 3.1; see also [25], Theorem 2.16, for an analogous result in the setting of invertible matrices.

For any allowable matrix $\mathfrak{m}$, now define $\boldsymbol{i}(\mathfrak{m}):=\inf _{x \in \mathbb{S}_{+}^{d-1}}|\mathfrak{m} x|$.

Hypothesis $\left(\mathrm{H}_{2}\right)$. There exists an $\alpha>0$ such that $\lambda(\alpha)=1$, and the following moment conditions hold:

$$
\mathbb{E}\left[\|M\|^{\alpha} \max \{|\log \|M\||,|\log \boldsymbol{i}(M)|\}\right]<\infty \quad \text { and } \quad \mathbb{E}\left[|Q|^{\alpha}\right]<\infty .
$$


The shifted distribution. We shall utilize the constant $\alpha$ in $\left(\mathrm{H}_{2}\right)$ to employ a change of measure, as developed in the multidimensional framework by Kesten [28]. Namely for $\mathfrak{m} \in \mathfrak{M}, \theta \in \mathfrak{D}$ and any $n \in \mathbb{N}_{+}$, define

$$
p_{n}^{\theta}(x, \mathfrak{m})=\frac{|\mathfrak{m} x|^{\theta}}{(\lambda(\theta))^{n}} \frac{r_{\theta}(\widetilde{\mathfrak{m} x})}{r_{\theta}(x)}, \quad x \in \mathbb{S}_{+}^{d-1} .
$$

Note by an application of Lemma 2.2 that

$$
\int p_{n}^{\theta}\left(x, \mathfrak{m}_{n} \cdots \mathfrak{m}_{1}\right) \mu^{\otimes n}\left(\left\{d \mathfrak{m}_{i}, d q_{i}\right\}_{i=1}^{n}\right)=1, \quad x \in \mathbb{S}_{+}^{d-1} .
$$

Moreover, the system of probability measures $\mu_{n, x}^{\theta}=p_{n}^{\theta}(x, \cdot) \mu^{\otimes n}$ is a projective system; hence by the Kolmogorov extension theorem, there exists a unique probability measure $\mathbb{P}_{x}^{\theta}$ on $\left(\mathfrak{M} \times \mathbb{R}_{+}^{d}\right)^{\mathbb{N}_{+}}$having marginals $\mu_{n, x}^{\theta}$. When the random variables $\left\{\left(M_{n}, Q_{n}\right): n=1,2, \ldots\right\}$ are generated by the measure $\mathbb{P}_{x}^{\theta}$ rather than the true underlying probability measure, we write $\mathbb{E}_{x}^{\theta}[\cdot]$. We shall refer to this measure as the " $\theta$-shifted measure."

It is worth observing that, although $\left\{\left(M_{n}, Q_{n}\right): n=1,2, \ldots\right\}$ is assumed to be i.i.d. in the unshifted measure, this sequence will be Markov-dependent in the $\theta$ shifted measure, for any $\theta>0$. However,

$$
\eta_{\theta}(E):=\int_{E} r_{\theta}(x) l_{\theta}(d x) \text { yields that } \widehat{\mathbb{P}}^{\theta}:=\int_{\mathbb{S}_{+}^{d-1}} \mathbb{P}_{x}^{\theta} \eta_{\theta}(d x)
$$

is shift-invariant, that is, the sequence $\left\{\left(M_{n}, Q_{n}\right)\right\}$ is stationary under $\widehat{\mathbb{P}}^{\theta}$; cf. Section 3.1 of [10]. This is an important observation, as it will allow us to apply the results of Hennion [26] on products of random matrices; cf. Section 4 below. Furthermore, by Lemma 6.2 of [10], $\mathbb{P}_{x}^{\theta} \ll \widehat{\mathbb{P}}^{\theta}$ for all $x \in \mathbb{S}_{+}^{d-1}$; and we shall use this result frequently to infer convergence $\mathbb{P}_{x}^{\alpha}$-a.s., for arbitrary $x \in \mathbb{S}_{+}^{d-1}$, by proving $\widehat{\mathbb{P}}^{\theta}$-a.s. convergence.

In the $\theta$-shifted measure, the limit behavior is described through the following generalization of the Furstenberg-Kesten theorem, which may be deduced from [26], Theorem 2, together with [10], Theorem 6.1.

LEMMA 2.3. Assume that $\left(\mathrm{H}_{1}\right)$ is satisfied and let $\theta \in \mathfrak{D}$, and suppose that $\left(\mathrm{H}_{2}\right)$ holds with $\theta$ in place of $\alpha$. Then for $x, y \in \mathbb{S}_{+}^{d-1}$, we have $\mathbb{P}^{\theta}$-a.s. that

$$
\begin{aligned}
& \lim _{n \rightarrow \infty} \frac{1}{n} \log \left|M_{n} \cdots M_{1} x\right|=\lim _{n \rightarrow \infty} \frac{1}{n} \log \left\|M_{n} \cdots M_{1}\right\|=\Lambda^{\prime}(\theta)=\hat{\mathbb{E}}^{\theta}\left[S_{1}\right] ; \\
& \lim _{n \rightarrow \infty} \sup \left\{\left|\frac{1}{n} \mathbf{1}_{\{\mathfrak{N} \leq n\}} \log \left\langle y, M_{n} \cdots M_{1} x\right\rangle-\Lambda^{\prime}(\theta)\right|: x, y \in \mathbb{S}_{+}^{d-1}\right\}=0 .
\end{aligned}
$$

Here, $\Lambda^{\prime}(\theta)$ is interpreted as a one-sided derivative if $\theta \in \partial \mathfrak{D}$. Note $\Lambda^{\prime}(0)<$ 0 , since $\Lambda$ is convex and $\Lambda(0)=\Lambda(\alpha)=1$; thus, the top Lyapunov exponent associated with $\left\{M_{n}\right\}$ is negative. Together with the moment assumptions in $\left(\mathrm{H}_{2}\right)$, 
this guarantees the existence of a unique stationary distribution for $\left\{V_{n}\right\}$ (cf. [28]), which is given by the law of

$$
V:=Q_{1}+\sum_{k=2}^{\infty} M_{1} \cdots M_{k-1} Q_{k} .
$$

The Markov random walk. The process $\left\{M_{n}\right\}$ induces a Markov random walk on $\mathbb{S}_{+}^{d-1} \times \mathbb{R}$, obtained by setting

$$
X_{n}=\left(M_{n} \cdots M_{1} X_{0}\right)^{\sim}, \quad S_{n}=\log \left|M_{n} \cdots M_{1} X_{0}\right|, \quad n=1,2, \ldots,
$$

for some initial state $X_{0} \in \mathbb{S}_{+}^{d-1}$ and $S_{0}=0$. In contrast to (1.3), in some contexts we will need to take $X_{0}$ to be different from $V_{0}$, but still independent of $\left\{\left(M_{n}, Q_{n}\right)\right\}$. This process will play an important role in the sequel. Note that in the $\theta$-shifted measure, $\left\{X_{n}\right\}$ has a unique stationary distribution given by the measure $\eta_{\theta}$ in (2.3); see [10], Theorem 4.11.

Probability measures. We introduce the following conventions to describe conditional probabilities which depend on the initial values of $X_{0}$ and $V_{0}$. Write

$$
\mathbb{P}_{v}(\cdot)=\mathbb{P}\left(\cdot \mid V_{0}=v\right), \quad \mathbb{P}_{x}^{\theta}(\cdot)=\mathbb{P}\left(\cdot \mid X_{0}=x\right), \quad \mathbb{P}_{x, v}^{\theta}(\cdot)=\mathbb{P}_{x}^{\theta}\left(\cdot \mid V_{0}=v\right),
$$

and use the same notation for the corresponding expectations. When conditioning on an initial distribution $V_{0} \sim \gamma$, write $\mathbb{P}_{\gamma}(\cdot)=\int \mathbb{P}_{v}(\cdot) \gamma(d v), \mathbb{P}_{\gamma}^{\theta}(\cdot)=$ $\int \mathbb{P}_{\tilde{v}, v}^{\theta}(\cdot) \gamma(d v)$, and finally set $\mathbb{P}_{\boldsymbol{\delta}_{v}}^{\theta}(\cdot)=\mathbb{P}_{\tilde{v}, v}^{\theta}(\cdot)$. We note that while working in the $\theta$-shifted measure, we will generally need to specify both $X_{0}$ and $V_{0}$ in these equations, and we will typically take $X_{0}=\widetilde{V}_{0}$. The reason for the asymmetry comes from the observation that, due to the Markov dependence in the $\theta$-shifted measure, the initial state does affect the law of $\left\{M_{n}\right\}$, and hence that of $\left\{V_{n}\right\}$ under $\mathbb{P}^{\theta}$. Finally, we note that we will sometimes suppress the dependence on $(x, v)$ when these values are clear and simply write $\mathbb{P}^{\alpha}$-a.s.

In this terminology, the change of measure can be written as follows: for all $n \in \mathbb{N}_{+}, x \in \mathbb{S}_{+}^{d-1}$, and any bounded measurable function $f: \mathbb{S}_{+}^{d-1} \times\left(\mathfrak{M} \times \mathbb{R}_{+}^{d}\right)^{n}$,

$$
\begin{aligned}
& r_{\alpha}(x) \mathbb{E}_{x, v}^{\alpha}\left[\frac{e^{-\alpha S_{n}}}{r_{\alpha}\left(X_{n}\right)} f\left(X_{0}, V_{0}, M_{1}, Q_{1}, \ldots, M_{n}, Q_{n}\right)\right] \\
& =\mathbb{E}\left[f\left(x, v, M_{1}, Q_{1}, \ldots, M_{n}, Q_{n}\right)\right] .
\end{aligned}
$$

2.3. Tail estimates for $\left\{V_{n}\right\}$. We now turn to our first main result, where we revisit and extend Kesten's well-known theorem in [28].

Let $\pi$ denote the stationary distribution of $\left\{V_{n}\right\}$, which is given by the law of the random variable $V$ defined in (2.4). Now fix a set $\mathbb{D} \subset \mathbb{R}_{+}^{d}$ where $\pi(\mathbb{D})>0$, and let $\pi_{\mathbb{D}}$ denote the stationary distribution of $\left\{V_{n}\right\}$ restricted to $\mathbb{D}$, that is,

$$
\pi_{\mathbb{D}}(E)=\frac{\pi(E \cap \mathbb{D})}{\pi(\mathbb{D})}, \quad E \in \mathscr{B}\left(\mathbb{R}_{+}^{d}\right) .
$$


Also let $\tau$ denote the first return time of $\left\{V_{n}\right\}$ to $\mathbb{D}$, namely,

$$
\tau=\inf \left\{n \in \mathbb{N}_{+}: V_{n} \in \mathbb{D}\right\} .
$$

Next, let $\overrightarrow{1}=(1, \ldots, 1)^{T}$, and define

$$
Y_{i}=\lim _{n \rightarrow \infty}\left(M_{i}^{\top} \cdots M_{n}^{\top \overrightarrow{1}}\right)^{\sim}, \quad n=1,2, \ldots
$$

Note that if $\theta \in \mathfrak{D}$, then the limit on the right-hand side exists $\mathbb{P}^{\theta}$-a.s., since this product constitutes a backward sequence of an iterated function system and the maps $\left\{M_{n}\right\}$ act as contractions on $\mathbb{S}_{+}^{d-1}$; cf. [26], Section 3. Moreover, the law of $Y_{i}$ is given by

$$
\eta_{\theta}^{*}(E):=\int_{E} r_{\theta}^{*}(x) l_{\theta}^{*}(d x), \quad E \in \mathscr{B}\left(\mathbb{S}_{+}^{d-1}\right),
$$

where $r_{\theta}^{*}$ and $l_{\theta}^{*}$ are given as in Lemma 2.2 (cf. [25], Theorem 3.2; [10], Proposition 3.1).

The condition $(\mathfrak{K})$. Recall that under $\left(\mathrm{H}_{1}\right)$, the measure $\mu_{M}$ is nonarithmetic, and hence $M_{n} \cdots M_{1}$ is positively regular for sufficiently large $n$ w.p.1, implying that for some positive integer $k$ and some $s>0$,

$$
M_{k} \cdots M_{2} Q_{1} \succ s \overrightarrow{1} \quad \text { with positive probability. }
$$

Now if $k>1$, then it is natural to introduce the $k$-step process; namely, fix $k \in \mathbb{N}_{+}$, and for all $n \in \mathbb{N}_{+}$, set

$$
\widehat{M}_{n}:=M_{k n} \cdots M_{k(n-1)+1} \quad \text { and } \quad \widehat{Q}_{n}=\sum_{i=k(n-1)+1}^{k n} M_{k n} \cdots M_{i+1} Q_{i} .
$$

Note as a consequence of these definitions that

$$
V_{k n}=\widehat{M}_{n} V_{k(n-1)}+\widehat{Q}_{n}, \quad n=1,2, \ldots,
$$

where $\widehat{Q}_{n}-s \overrightarrow{1} \succ 0$ with positive probability. It is worth observing here that the stationary distributions of $\left\{V_{k n}\right\}$ and $\left\{V_{n}\right\}$ are, of course, identical.

Finally, let $\mathscr{C}_{0}\left(\mathbb{R}_{+}^{d} \backslash\{0\}\right)$ denote the set of bounded continuous functions on $\mathbb{R}_{+}^{d} \backslash\{0\}$ which are supported on $\mathbb{R}_{+}^{d} \backslash B_{r}(0)$, for some $r>0$.

THEOREM 2.4. Assume that Hypotheses $\left(\mathrm{H}_{1}\right)$ and $\left(\mathrm{H}_{2}\right)$ are satisfied, and suppose that $\mathbb{D}=B_{r}^{+}(0)$, where $r$ has been chosen sufficiently large such that $\pi(\mathbb{D})>0$. If $f \in \mathscr{C}_{0}\left(\mathbb{R}_{+}^{d} \backslash\{0\}\right)$ and $k=1$ in $(\mathfrak{K})$, then

$$
\lim _{u \rightarrow \infty} u^{\alpha} \mathbb{E}\left[f\left(\frac{V}{u}\right)\right]=\frac{C}{\lambda^{\prime}(\alpha)} \int_{\mathbb{S}_{+}^{d-1} \times \mathbb{R}} e^{-\alpha s} f\left(e^{s} x\right) l_{\alpha}(d x) d s,
$$


where

$$
C=\int_{\mathbb{D}} r_{\alpha}(\widetilde{v}) \mathbb{E}_{\delta_{v}}^{\alpha}\left[\left(|v|+\sum_{i=1}^{\infty} \frac{\left\langle Y_{i}, \widetilde{Q}_{i}\right\rangle}{\left\langle Y_{i}, X_{i}\right\rangle} \frac{\left|Q_{i}\right|}{\left|M_{i} \cdots M_{1} \widetilde{v}\right|}\right)^{\alpha} \mathbf{1}_{\{\tau=\infty\}}\right] \pi(d v) .
$$

If $k>1$ in $(\mathfrak{K})$, then the theorem still holds, but the constant $C$ is then computed with respect to the $k$-step chain $\left\{V_{k n}\right\}$ generated by $\left\{\left(\widehat{M}_{i}, \widehat{Q}_{i}\right)\right\}$ rather than with respect to the 1-step chain $\left\{V_{n}\right\}$.

If $\left\{V_{n}\right\}$ is a Harris recurrent chain, then we may always take $k=1$; see Proposition 5.2 below. Moreover, if $Q \succ 0$ with positive probability, then we may again take $k=1$.

More generally, when dealing with the $k$-step chain, we observe that the stopping time $\tau$ in (2.10) must now be computed with respect to that chain (rather than the 1-step chain), and the drift factor $\lambda^{\prime}(\alpha)$ in (2.9) must be replaced with the drift of the $k$-step chain, namely $k \lambda^{\prime}(\alpha)$; cf. Remark 5.3 below.

REMARK 2.5. For another representation of (2.9), let $\mathfrak{L}_{\alpha}$ be the measure on $\mathbb{R}_{+}^{d} \backslash\{0\}$ defined by the equation

$$
\int_{\mathbb{S}_{+}^{d-1} \times \mathbb{R}} e^{-\alpha s} f\left(e^{s} x\right) l_{\alpha}(d x) d s=\int_{\mathbb{R}_{+}^{d} \backslash\{0\}} f(x) \mathfrak{L}_{\alpha}(d x) .
$$

Then (2.9) gives the vague convergence (of measures on $\mathbb{R}_{+}^{d} \backslash\{0\}$ ) toward $C / \lambda^{\prime}(\alpha)$. In particular, for any measurable set $A \subset \mathbb{R}_{+}^{d}$ which is bounded away from zero and satisfies $\mathfrak{L}_{\alpha}(\partial A)=0$, it follows from the Portmanteau theorem that

$$
\lim _{u \rightarrow \infty} u^{\alpha} \mathbb{P}(V \in u A)=\frac{C}{\lambda^{\prime}(\alpha)} \mathfrak{L}_{\alpha}(A) .
$$

Furthermore, note that for any $t>0$ and any measurable $E \subset \mathbb{S}_{+}^{d-1}$ with $l_{\alpha}(\partial E)=0$, the sets $E^{t}:=\left\{x \in \mathbb{R}_{+}^{d}:|x|>t, x /|x| \in E\right\}$ are $\mathfrak{L}_{\alpha}$-continuous. Hence, for all $E \subset \mathbb{S}_{+}^{d-1}$ with $l_{\alpha}(\partial E)=0$,

$$
\lim _{u \rightarrow \infty} u^{\alpha} \mathbb{P}\left(|V|>t u, \frac{V}{|V|} \in E\right)=\frac{C}{\alpha \lambda^{\prime}(\alpha)} t^{-\alpha} l_{\alpha}(E) .
$$

Thus we infer the weak convergence

$$
\lim _{u \rightarrow \infty} \mathbb{P}\left(\frac{V}{|V|} \in \cdot|| V \mid>u\right) \Rightarrow l_{\alpha}(\cdot) .
$$

REMARK 2.6. Let $\widetilde{C}(v)$ denote the expectation in (2.10), that is, $C=$ $\int_{\mathbb{D}} r_{\alpha}(\tilde{v}) \widetilde{C}(v) \pi(d v)$. Then we have two further representations for $\widetilde{C}(v)$. First, by Lemma 3.6 below, it will follow that

$$
\widetilde{C}(v)=\lim _{n \rightarrow \infty} \mathbb{E}_{\delta_{v}}^{\alpha}\left[\left(\frac{\left|V_{n}\right|}{\left|M_{n} \cdots M_{1} \tilde{V}_{0}\right|}\right)^{\alpha} \mathbf{1}_{\{\tau \geq n\}}\right] .
$$


Moreover, it will follow by combining Corollary 4.2 [noting $C(v)=r_{\alpha}(\tilde{v}) \widetilde{C}(v)$ ] with Lemma 6.1 that

$$
\widetilde{C}(v)=\lim _{u \rightarrow \infty} \frac{\mathbb{P}\left(\left|V_{n}\right|>u, 0 \leq n<\tau \mid V_{0}=v\right)}{\mathbb{P}\left(S_{n}>\log u, \text { for some } n \in \mathbb{N} \mid X_{0}=\tilde{v}\right)} .
$$

The latter expression shows that $\widetilde{C}(v)$ describes the discrepancy between the probability of a large exceedance of $\left\{V_{n}\right\}$ occurring over a cycle, and the probability of ruin for the corresponding Markov random walk.

We conclude this section with a brief comparison of our result to some recent one-dimensional representations. As noted in the Introduction, (2.10) can be viewed as a generalization of a result of Collamore and Vidyashankar [18] to the multidimensional setting. Alternatively, building upon Goldie [23], it is shown in Buraczewski et al. [12] that in the one-dimensional setting (and its generalization to the class of similarities described there), we have $C=$ $\left(\alpha \lambda^{\prime}(\alpha)\right)^{-1} \lim _{n \rightarrow \infty} n^{-1} \mathbb{E}\left[\left|V_{n}\right|^{\alpha}\right]$. Finally, a further one-dimensional representation was derived in Enriquez et al. [21], expressed in terms of expectations of perpetuity sequences under a delicate conditioning on the process. The proofs of these one-dimensional results are all quite different and, therefore, it is not transparent how they can be easily unified.

2.4. Extremal estimates for maxima and first passage times. Our next objective is to study the probability of a large exceedance occurring over a single cycle emanating from, and then returning to, a given set $\mathbb{D} \subset \mathbb{R}_{+}^{d}$, and, in this way, to characterize the distribution of the first passage time

$$
T_{u}:=\inf \left\{n \in \mathbb{N}_{+}:\left|V_{n}\right|>u\right\},
$$

or more generally,

$$
T_{u}^{A}:=\inf \left\{n \in \mathbb{N}_{+}: V_{n} \in u A\right\} \quad \text { where } A \subset\left\{x \in \mathbb{R}_{+}^{d}:|x|>1\right\},
$$

and we assume that the set $A$ satisfies the following regularity property.

Definition 2.7. We say that a set $A \in \mathscr{B}\left(\mathbb{R}^{d}\right)$ is a semi-cone if $A \subset \mathbb{R}_{+}^{d} \backslash$ $B_{1}(0)$ and $x \in \partial A \Rightarrow\{t x: t>1\} \subset A$.

Now suppose that $A$ is a semi-cone, let $\left\{S_{n}\right\}$ be defined as in (2.5), and set

$$
\begin{aligned}
d_{A}(x) & =\inf \{t>1: t x \in A\}, \quad x \in \mathbb{S}_{+}^{d-1} ; \\
S_{n}^{A} & =S_{n}-\log d_{A}\left(X_{n}\right), \quad n=0,1,2, \ldots ; \\
r_{\alpha}^{A}(x) & =r_{\alpha}(x)\left(d_{A}(x)\right)^{\alpha}, \quad x \in \mathbb{S}_{+}^{d-1} ; \\
\mathfrak{P}_{A} & =\left\{x \in \mathbb{S}_{+}^{d-1}: d_{A}(x)<\infty\right\} .
\end{aligned}
$$


As a consequence of Kesten's renewal theorem, it will be shown in Lemma 6.1 below that if $\mathfrak{P}_{A}=\mathbb{S}_{+}^{d-1}$, then

$$
\mathbb{P}\left(M_{n} \cdots M_{1} \widetilde{V}_{0} \in u A \text {, for some } n \in \mathbb{N}_{+} \mid V_{0}=v\right) \sim r_{\alpha}(\widetilde{v}) D_{A} u^{-\alpha}
$$

as $u \rightarrow \infty$, where

$$
D_{A}:=\int_{\mathbb{S}_{+}^{d-1} \times \mathbb{R}_{+}} \frac{e^{-\alpha s}}{r_{\alpha}^{A}(x)} \varrho^{A}(d x, d s)
$$

and the measure $\varrho^{A}$ will be specified below in Section 3.3. Essentially, (2.19) is the ruin estimate for the Markov random walk $\left\{\left(X_{n}, S_{n}^{A}\right): n=0,1, \ldots\right\}$ under the initial state $X_{0}=\tilde{v}$, and $\varrho^{A}$ corresponds to the stationary excess distribution for this process. Indeed, if $A$ is a semi-cone and $d_{A}$ is continuous, then it follows immediately from the definitions that $e^{S_{n}} X_{n} \in u A \Leftrightarrow e^{S_{n}}>u \cdot d_{A}\left(X_{n}\right)$, and hence, on the left-hand side of (2.19),

$$
M_{n} \cdots M_{1} \tilde{V}_{0} \in u A \quad \Leftrightarrow \quad e^{S_{n}} X_{n} \in u A \quad \Leftrightarrow \quad S_{n}^{A}>\log u .
$$

Now if $\mathfrak{P}_{A} \neq \mathbb{S}_{+}^{d-1}$, then (2.19) will still hold and this defines the constant $D_{A}$, although the identification of $D_{A}$ is less explicit in that case [i.e., there is no equivalent of (2.20)]. However, $D_{A}$ can nonetheless be interpreted as the ruin constant for the Markov random walk; see Section 6 below.

Finally, let $C$ be defined as in (2.10), and set

$$
C(v)=r_{\alpha}(\widetilde{v}) \mathbb{E}_{\delta_{v}}^{\alpha}\left[\left(|v|+\sum_{i=1}^{\infty} \frac{\left\langle Y_{i}, \widetilde{Q}_{i}\right\rangle}{\left\langle Y_{i}, X_{i}\right\rangle} \frac{\left|Q_{i}\right|}{\left|M_{i} \cdots M_{1} \widetilde{v}\right|}\right)^{\alpha} \mathbf{1}_{\{\tau=\infty\}}\right]
$$

THEOREM 2.8. Suppose that Hypotheses $\left(\mathrm{H}_{1}\right)$ and $\left(\mathrm{H}_{2}\right)$ are satisfied and $\mathbb{D}=$ $B_{r}^{+}(0)$, where $r$ has been chosen sufficiently large such that $\pi(\mathbb{D})>0$. Assume that $A$ is a semi-cone and $d_{A}$ is continuous. Then for any $v \in \mathbb{R}_{+}^{d} \backslash\{0\}$,

$$
\lim _{u \rightarrow \infty} u^{\alpha} \mathbb{P}\left(T_{u}^{A}<\tau \mid V_{0}=v\right)=D_{A} C(v)
$$

Furthermore, assuming that $k=1$ in $(\mathfrak{K})$, we have that the normalized sequence $\left\{T_{u}^{A} / u^{\alpha}\right\}$ converges in distribution; more precisely,

$$
\lim _{u \rightarrow \infty} \mathbb{P}\left(\frac{T_{u}^{A}}{u^{\alpha}} \leq z \mid V_{0}=v\right)=1-e^{-K_{A} z}, \quad z \geq 0,
$$

for all $v \in \mathbb{R}_{+}^{d} \backslash\{0\}$, where $K_{A}=C D_{A}$.

As in Theorem 2.4, the assumption $k=1$ is not necessary if $\left\{V_{n}\right\}$ is Harris recurrent or if $Q \succ 0$ with positive probability. 
REMARK 2.9. For one-dimensional recursions, related estimates have previously been given for the distribution of $\max \left\{V_{i}: 1 \leq i \leq n^{1 / \alpha} u\right\}$ as $n \rightarrow \infty$; cf. [19], Theorem 2.1, or [41]. However, in the multidimensional setting, the only result we are aware of is that of Perfekt [42], who studies the componentwise maxima, namely

$$
\left(\max _{1 \leq i \leq n} V_{1}, \ldots, \max _{1 \leq i \leq n} V_{d}\right) \quad \text { as } n \rightarrow \infty
$$

Note that the componentwise maxima need not be achieved simultaneously; hence Perfekt's results do not coincide with ours. Moreover, in all of these references, additional conditions are assumed which we do not impose here; in particular, in their formulations it must be assumed that $V_{0} \sim \pi$.

REMARK 2.10. As a particular application of the previous theorem, we now determine the extremal index of $\left\{\left|V_{n}\right|\right\}$. Integrating with respect to the measure $\pi$ in (2.23), we obtain that

$$
\lim _{u \rightarrow \infty} \mathbb{P}\left(\frac{T_{u}^{A}}{u^{\alpha}} \leq z \mid V_{0} \sim \pi\right)=1-e^{-K_{A} z}, \quad z \geq 0 .
$$

Set $A=\{x:|x|>1\}$. Then it easily follows with $u=n^{1 / \alpha} w$ and $z=w^{-\alpha}$ that

$$
\lim _{n \rightarrow \infty} \mathbb{P}\left(\max _{1 \leq i \leq n}\left|V_{i}\right| \leq n^{1 / \alpha} w \mid V_{0} \sim \pi\right)=e^{-K_{A} w^{-\alpha}}
$$

Moreover, for this choice of $A$, it follows by Theorem 2.4 that

$$
\lim _{n \rightarrow \infty} n \mathbb{P}\left(|V|>n^{1 / \alpha} w\right)=\frac{C}{\alpha \lambda^{\prime}(\alpha)} w^{-\alpha} .
$$

Then reasoning as in [31], Section 2.2, we conclude from (2.24) and (2.25) that the extremal index of $\left\{\left|V_{n}\right|\right\}$ is given by

$$
\Theta=\alpha \lambda^{\prime}(\alpha) D_{A} .
$$

For a related result in the one-dimensional setting, see [18], Proposition 2.2.

2.5. The path and empirical law under a large exceedance. We conclude by examining the path behavior of $\left\{V_{n}\right\}$ prior to a large exceedance. Motivated by classical results for random walk (e.g., Section XII.6.(d) of [22] or more recent work in [5,7]), it is natural to expect that, conditioned on $\left\{T_{u}^{A}<\tau\right\}$ (where $\tau$ is the return time to any $\pi$-positive set $\mathbb{D}),\left\{V_{n}\right\}$ should behave as its "associate," which, in our setting, translates to the process $\left\{e^{S_{n}} X_{n}\right\}$ under the $\alpha$-shifted measure.

However, in our problem, we cannot anticipate that the behavior of $\left\{V_{n}\right\}$ will mimic that of $\left\{e^{S_{n}} X_{n}\right\}$ over the entire trajectory. For this reason, we introduce an "initial" level $\varepsilon_{u}$, where $\varepsilon_{u}=o(u)$ and $\varepsilon_{u} \uparrow \infty$ as $u \rightarrow \infty$, and study the trajectory of $\left\{V_{n}\right\}$ subsequent to its exceedance over the level $\varepsilon_{u}$. 
THEOREM 2.11. Suppose that Hypotheses $\left(\mathrm{H}_{1}\right)$ and $\left(\mathrm{H}_{2}\right)$ are satisfied, and assume that $A$ is a semi-cone and the function $d_{A}$ is bounded and continuous on $\mathbb{S}_{+}^{d-1}$. Let $m \in \mathbb{N}_{+}$, and let $g:\left(\mathbb{R}_{+}^{d}\right)^{m+1} \rightarrow \mathbb{R}$ be $\theta$-Hölder continuous for some $\theta \leq \min \{1, \alpha\}$ and also bounded. Set

$$
I_{u}=T_{\varepsilon_{u}} \quad \text { where } \varepsilon_{u}=o(u) \text { and } \varepsilon_{u} \nearrow \infty \text { as } u \rightarrow \infty .
$$

Then for all $v \in \mathbb{R}_{+}^{d}$,

$$
\begin{aligned}
\lim _{u \rightarrow \infty} & \mathbb{E}_{v}\left[g\left(\frac{V_{I_{u}}}{\left|V_{I_{u}}\right|}, \ldots, \frac{V_{I_{u}+m}}{\left|V_{I_{u}}\right|}\right) \mid T_{u}^{A}<\tau\right] \\
& =\int_{\mathbb{S}_{+}^{d-1} \times \mathbb{R}_{+}} \mathbb{E}_{x}^{\alpha}\left[g\left(X_{0}, e^{S_{1}} X_{1}, \ldots, e^{S_{m}} X_{m}\right)\right] \varrho(d x, d s) .
\end{aligned}
$$

The class of $\theta$-Hölder continuous functions is a separating class, and thus for all $m \in \mathbb{N}_{+}$, we then deduce the weak convergence

$$
\begin{aligned}
& \mathbb{P}\left(\left(\frac{V_{I_{u}}}{\left|V_{I_{u}}\right|}, \ldots, \frac{V_{I_{u}+m}}{\left|V_{I_{u}}\right|}\right) \in \cdot \mid T_{u}^{A}<\tau\right) \\
& \quad \Rightarrow \quad \int_{\mathbb{S}_{+}^{d-1} \times \mathbb{R}_{+}} \mathbb{P}_{x}^{\alpha}\left(\left(X_{0}, e^{S_{1}} X_{1}, \ldots, e^{S_{m}} X_{m}\right) \in \cdot\right)(d x, d s),
\end{aligned}
$$

for any given $V_{0}$.

Finally, we conclude by studying the empirical law of $\left\{\log \left|V_{n}\right|-\log \left|V_{n-1}\right|\right\}$.

THEOREM 2.12. Suppose that Hypotheses $\left(\mathrm{H}_{1}\right)$ and $\left(\mathrm{H}_{2}\right)$ are satisfied, and assume that $A$ is a semi-cone and $d_{A}$ is bounded and continuous on $\mathbb{S}_{+}^{d-1}$. Then for any $v \in \mathbb{R}_{+}^{d}$ and any bounded Lipschitz continuous function $g: \mathbb{R} \rightarrow \mathbb{R}$,

$$
\lim _{u \rightarrow \infty} \mathbb{E}_{v}\left[\left|\frac{1}{T_{u}^{A}} \sum_{n=1}^{T_{u}^{A}} g\left(\log \left(\frac{\left|V_{n}\right|}{\left|V_{n-1}\right|}\right)\right)-\widehat{\mathbb{E}}^{\alpha}\left[g\left(S_{1}\right)\right]\right| \mid T_{u}^{A}<\tau\right]=0 .
$$

Thus the empirical law of $\left\{\left(\log \left|V_{n}\right|-\log \left|V_{n-1}\right|\right)\right\}$ converges weakly, in $\mathbb{P}_{v}\left(\cdot \mid T_{u}^{A}<\tau\right)$-probability, to $\widehat{\mathbb{P}}^{\alpha}\left(S_{1} \in \cdot\right)$.

By comparing with [10], Theorem 6.1, we see that (2.28) agrees precisely with the empirical law, without conditioning, of the Markov random walk $\left\{\left(X_{n}, S_{n}\right)\right\}$ under the $\alpha$-shifted measure.

\section{Background.}

3.1. Preliminary results from Markov chain theory. We start by deriving an analog of the drift condition from Markov chain theory. 
LEMMA 3.1. Assume that $\left(\mathrm{H}_{1}\right)$ and $\left(\mathrm{H}_{2}\right)$ are satisfied. Then for any $0<\theta<$ $\min \{1, \alpha\}$, there exist positive constants $t<1$ and $L<\infty$ such that for $\mathbb{D}^{\dagger}:=\{v \in$ $\left.\mathbb{R}_{+}^{d}:|v| \leq L\right\}$

$$
\mathbb{E}\left[\left|V_{n}\right|^{\theta} r_{\theta}\left(\tilde{V}_{n}\right) \mid \mathscr{F}_{n-1}\right] \leq t\left|V_{n-1}\right|^{\theta} r_{\theta}\left(\tilde{V}_{n-1}\right) \quad \text { for all } V_{n-1} \in \mathbb{R}_{+}^{d} \backslash \mathbb{D}^{\dagger}
$$

In particular, for $\tau^{\dagger}:=\inf \left\{n \in \mathbb{N}_{+}: V_{n} \in \mathbb{D}^{\dagger}\right\}$, there exists $B<\infty$ such that

$$
\mathbb{E}\left[\left|V_{n}\right|^{\theta} \mathbf{1}_{\left\{\tau^{\dagger}>n\right\}} \mid V_{0}=v\right] \leq B t^{n}|v|^{\theta} \quad \text { for all } v \in \mathbb{R}_{+}^{d} \backslash \mathbb{D}^{\dagger} .
$$

ProOF. Let $\theta \in(0,1)$. By applying equation (2.2) of Lemma 2.2, then using subadditivity and a further application of (2.2), we obtain that for some $c \in(0, \infty)$,

$$
\begin{aligned}
& \mathbb{E}\left[\left|V_{n}\right|^{\theta} r_{\theta}\left(\widetilde{V}_{n}\right) \mid \mathscr{F}_{n-1}\right] \\
& \quad=c \mathbb{E}\left[\int_{\mathbb{S}_{+}^{d-1}}\left\langle y, V_{n}\right\rangle^{\theta} l_{\theta}^{*}(d y) \mid \mathscr{F}_{n-1}\right] \\
& \quad \leq c \mathbb{E}\left[\int_{\mathbb{S}_{+}^{d-1}}\left(\left\langle y, M_{n} V_{n-1}\right\rangle^{\theta}+\left\langle y, Q_{n}\right\rangle^{\theta}\right) l_{\theta}^{*}(d y) \mid V_{n-1}\right] \\
& \quad \leq \mathbb{E}\left[\left|M_{n} V_{n-1}\right|^{\theta} r_{\theta}\left(\left(M_{n} V_{n-1}\right)^{\sim}\right) \mid V_{n-1}\right]+\mathbb{E}\left[\left|Q_{n}\right|^{\theta}\right] .
\end{aligned}
$$

The first term on the right-hand side equals

$$
\left|V_{n-1}\right|^{\theta} P_{\theta} r_{\theta}\left(\tilde{V}_{n-1}\right)=\left|V_{n-1}\right|^{\theta} \lambda(\theta) r_{\theta}\left(\tilde{V}_{n-1}\right),
$$

and so the required estimate follows under Hypothesis $\left(\mathrm{H}_{2}\right)$, choosing $\theta$ such that $0<\theta<\min \{\alpha, 1\}$.

Using that $r_{\theta}$ is bounded from above and below by finite positive constants (by Lemma 2.2), (3.2) is then obtained by iterating (3.1).

LEMMA 3.2. Suppose $\left(\mathrm{H}_{1}\right)$ and $\left(\mathrm{H}_{2}\right)$ are satisfied, and let $\mathbb{D}=B_{r}^{+}(0)$ for some $r>0$ such that $\pi(\mathbb{D})>0$. Let $\mathbb{D}^{\dagger}=\left\{v \in \mathbb{R}_{+}^{d}:|v| \leq L\right\}$, where $L$ is chosen such that (3.1) is satisfied and such that $\mathbb{D}^{\dagger} \supset \mathbb{D}$. Then there exist constants $t \in$ $(0,1)$ and $B<\infty$ such that for $\tau=\inf \left\{n \in \mathbb{N}_{+}: V_{n} \in \mathbb{D}\right\}$,

$$
\sup _{v \in \mathbb{D}^{\dagger}} \mathbb{P}\left(\tau>n \mid V_{0}=v\right) \leq B t^{n} \quad \text { for all } n \in \mathbb{N}_{+} \text {. }
$$

Proof. From (3.1), it follows that, starting from an initial state $V_{0} \notin \mathbb{D}^{\dagger},\left\{V_{n}\right\}$ returns to $\mathbb{D}^{\dagger}$ at a geometric rate; for a proof, see [37], Theorem 15.2.5. Thus it suffices to show that

$$
\sup _{v \in \mathbb{D}^{\dagger}} \mathbb{P}\left(\tau>n \mid V_{0}=v\right) \leq(1-s)
$$

for some $s>0$ and $n \in \mathbb{N}_{+}$. To establish (3.5), we use Proposition 4.3.1 of [11], which precisely describes supp $\pi$. Namely, there exists a set $\mathscr{S}$ with $\overline{\mathscr{S}}=$ 
$\operatorname{supp} \pi$ such that the following holds: For each $v_{0} \in \mathscr{S}$, there exists $l \in \mathbb{N}_{+}$and $\mathfrak{m}_{1}, \ldots, \mathfrak{m}_{l} \in \operatorname{supp} \mu_{M}, q_{1}, \ldots, q_{l} \in \operatorname{supp} \mu_{Q}$ such that

$$
h: v \mapsto \mathfrak{m}_{l} \cdots \mathfrak{m}_{1} v+\sum_{i=1}^{l} \mathfrak{m}_{l} \cdots \mathfrak{m}_{i+1} q_{i}
$$

is a contraction on $\mathbb{R}_{+}^{d}$ with $v_{0}$ as the unique fixed point. Hence, using that $\mathbb{D}^{\dagger}$ is compact, we obtain that for any $\delta>0$, there exists $j \in \mathbb{N}_{+}$such that $\left|h^{j}(v)-v_{0}\right|<$ $\delta / 2$ for all $v \in \mathbb{D}^{\dagger}$. Then, from continuity and the definition of the support, we conclude that

$$
\inf _{v \in \mathbb{D}^{\dagger}} \mathbb{P}\left(\left|V_{l j}-v_{0}\right|<\delta \mid V_{0}=v\right)>0 .
$$

Since $\mathbb{D}$ is open in $\mathbb{R}_{+}^{d}$ and $\pi(\mathbb{D})>0$, and hence $\mathbb{D} \cap \operatorname{supp} \pi \neq \varnothing$, it follows that $\mathbb{D} \cap \mathscr{S} \neq \varnothing$ as well. Now let $v_{0} \in \mathbb{D} \cap \mathscr{S}$ and choose $\delta>0$ such that $B_{\delta}\left(v_{0}\right) \in \mathbb{D}$. Then (3.5) follows from (3.6) with $k=l j$.

For an arbitrary $\pi$-positive set $\mathbb{D}$, define the return times $\kappa_{0}=0$ and

$$
\kappa_{i}=\inf \left\{n>\kappa_{i-1}: V_{n} \in \mathbb{D}\right\}, \quad i=1,2, \ldots,
$$

and let $\tau_{i}:=\kappa_{i}-\kappa_{i-1}$. Set $N_{\mathbb{D}}(n)=\sum_{k=1}^{n} \mathbf{1}_{\mathbb{D}}\left(V_{k}\right)$. Using that $\left\{V_{n}\right\}$ is stationary and ergodic when $V_{0} \sim \pi$, we infer the following strong law of large numbers for $\left\{\kappa_{i}\right\}$, which is standard.

LEMMA 3.3. Suppose that $\left(\mathrm{H}_{1}\right)$ and $\left(\mathrm{H}_{2}\right)$ are satisfied and $\pi(\mathbb{D})>0$. Then for $\pi$-a.e. $v \in \mathbb{R}_{+}^{d}$,

$$
\lim _{i \rightarrow \infty} \frac{\kappa_{i}}{i}=\lim _{n \rightarrow \infty}\left(\frac{N_{\mathbb{D}}(n)}{n}\right)^{-1}=\frac{1}{\pi(\mathbb{D})}=\mathbb{E}_{\pi_{\mathbb{D}}}\left[\tau_{1}\right] \quad \mathbb{P}_{v} \text {-a.s. }
$$

and $\pi_{\mathbb{D}}(\cdot):=\pi(\cdot) / \pi(\mathbb{D})$ is invariant for the process $\left\{V_{\kappa_{i}}: i=0,1, \ldots\right\}$.

Now let $P$ denote the transition kernel of $\left\{V_{n}\right\}$. We conclude this section with two results which hold under the following additional Hypothesis $\left(\mathrm{H}_{3}\right)$ (which will ultimately be dropped in our main theorems by utilizing a smoothing argument).

HYPOTHESIS $\left(\mathrm{H}_{3}\right)$. Assume the following conditions:

(i) There exists a $\pi$-positive set $F$ such that, for each $v \in F, P(v, \cdot)$ has an absolutely continuous component with respect to some $\sigma$-finite nonnull measure $\Phi$.

(ii) $(\operatorname{supp} \pi)^{\circ} \neq \varnothing$.

Note that under $\left(\mathrm{H}_{3}\right)$, it follows from [3], Theorem 2.1(b) and Theorem 2.2(b) that $\left\{V_{n}\right\}$ is an aperiodic, positive Harris chain on $\mathbb{R}_{+}^{d}$. Once this is observed, the following result is also standard; cf. [37], Theorem 15.0.1. 
LEMMA 3.4. Assume that $\left(\mathrm{H}_{1}\right),\left(\mathrm{H}_{2}\right)$ and $\left(\mathrm{H}_{3}\right)$ are satisfied. Then $\left\{V_{n}\right\}$ is an aperiodic, positive Harris chain on $\mathbb{R}_{+}^{d}$. Moreover, $\left\{V_{n}\right\}$ is $\psi$-irreducible, regular and geometrically recurrent.

LEMMA 3.5. Suppose that $\left(\mathrm{H}_{1}\right),\left(\mathrm{H}_{2}\right)$ and $\left(\mathrm{H}_{3}\right)$ are satisfied, and let $\mathbb{D} \subset \mathbb{R}_{+}^{d}$ be chosen such that $\pi(\mathbb{D})>0$. Let $\tau:=\inf \left\{n \in \mathbb{N}_{+}: V_{n} \in \mathbb{D}\right\}$ denote the first return time of $\mathbb{D}$. Then for any $\pi$-integrable function $h$,

$$
\int h(v) \pi(d v)=\mathbb{E}[h(V)]=\frac{1}{\mathbb{E}_{\pi_{\mathbb{D}}}[\tau]} \mathbb{E}_{\pi_{\mathbb{D}}}\left[\sum_{i=0}^{\tau-1} h\left(V_{i}\right)\right] .
$$

Proof. See [40], Proposition 5.9 and the discussion just prior to [40], Corollary 5.3. For a closely related result, see the proof of [15], Theorem 2.1.

3.2. Quantifying the discrepancy between $\left\{V_{n}\right\}$ and $\left\{e^{S_{n}} X_{n}\right\}$. Set

$$
Z_{n}=\frac{V_{n}}{\left|M_{n} \cdots M_{1} X_{0}\right|} \quad \text { and } \quad Z_{n}^{(0)}=\frac{\sum_{i=1}^{n} M_{n} \cdots M_{i+1} Q_{i}}{\left|M_{n} \cdots M_{1} X_{0}\right|}
$$

for all $n \in \mathbb{N}$. [Thus $Z_{n}^{(0)}=\left(V_{n}-V_{0}\right) /\left|M_{n} \cdots M_{1} X_{0}\right|$.] Also introduce the shorthand notation

$$
\Pi_{n}:=M_{n} \cdots M_{1} \quad \text { and } \quad \Pi_{i}^{n}:=M_{n} \cdots M_{i}
$$

LEMma 3.6. Assume $\left(\mathrm{H}_{1}\right)$ and $\left(\mathrm{H}_{2}\right)$. Then:

(i) $\sup _{n \in \mathbb{N}}\left|Z_{n}\right|<\infty \mathbb{P}^{\alpha}$-a.s. and $\sup _{n \in \mathbb{N}}\left|Z_{n}^{(0)}\right|<\infty \mathbb{P}^{\alpha}$-a.s.

(ii) Suppose $v \in \mathbb{R}_{+}^{d} \backslash\{0\}$. Then in $\mathbb{P}_{\delta_{v}^{\alpha}}^{\alpha}$-measure, the sequence $\left\{Z_{n}\right\}$ converges in law to a random variable $Z$, and $\left|Z_{n}\right| \Rightarrow|Z|$ a.s., where

$$
|Z|=|v|+\sum_{i=1}^{\infty} \frac{\left\langle Y_{i}, \widetilde{Q}_{i}\right\rangle}{\left\langle Y_{i}, X_{i}\right\rangle} \frac{\left|Q_{i}\right|}{\left|\Pi_{i} \widetilde{v}\right|} \quad \mathbb{P}_{\boldsymbol{\delta}_{v}}^{\alpha} \text {-a.s. }
$$

Moreover, $|Z|$ is strictly positive and finite $\mathbb{P}_{\delta_{v}}^{\alpha}$-a.s. Similarly,

$$
\lim _{n \rightarrow \infty}\left|Z_{n}^{(0)}\right|=\sum_{i=1}^{\infty} \frac{\left\langle Y_{i}, \widetilde{Q}_{i}\right\rangle}{\left\langle Y_{i}, X_{i}\right\rangle} \frac{\left|Q_{i}\right|}{\left|\Pi_{i} \widetilde{v}\right|} \quad \mathbb{P}_{\boldsymbol{\delta}_{v}}^{\alpha} \text {-a.s. }
$$

(iii) Let $F \subset \mathbb{R}_{+}^{d} \backslash\{0\}$ be a bounded set and let $\tau^{\prime}$ be any $\left\{\mathscr{F}_{n}\right\}$-stopping time such that $\sup _{v \in F} \mathbb{P}\left(\tau^{\prime}>k \mid V_{0}=v\right) \leq B t^{k}, k \in \mathbb{N}$, for some finite constant $B$ and $t \in(0,1)$. Then for any $v \in \mathbb{R}_{+}^{d} \backslash\{0\}$,

$$
\sup _{v \in F} \mathbb{E}_{\boldsymbol{\delta}_{v}}^{\alpha}\left[\sup _{n \in \mathbb{N}}\left|Z_{n}\right|^{\alpha} \mathbf{1}_{\left\{\tau^{\prime} \geq n\right\}}\right]<\infty \quad \text { and } \quad \sup _{v \in F} \mathbb{E}_{\boldsymbol{\delta}_{v}}^{\alpha}\left[|Z|^{\alpha} \mathbf{1}_{\left\{\tau^{\prime}=\infty\right\}}\right]<\infty
$$


(iv) For $v \in \mathbb{R}_{+}^{d} \backslash\{0\}$, we have the $L^{1}$-convergence

$$
\lim _{n \rightarrow \infty} \mathbb{E}_{\delta_{v}}^{\alpha}\left[\left|Z_{n}\right|^{\alpha} \mathbf{1}_{\left\{\tau^{\prime} \geq n\right\}}-|Z|^{\alpha} \mathbf{1}_{\left\{\tau^{\prime}=\infty\right\}} \mid\right]=0 .
$$

Note that by Lemma 3.2, the condition in (iii) holds, in particular, for $\tau^{\prime}=\tau:=$ $\inf \left\{n \in \mathbb{N}_{+}: V_{n} \in \mathbb{D}\right\}$ with $F=\mathbb{D} \backslash\{0\}$.

Proof of Lemma 3.6. For any vector $x \in \mathbb{R}^{d}$, let $x^{(i)}=\left\langle e_{i}, x\right\rangle$ denote the $i$ th component of $x$, and set $\overrightarrow{1}=(1, \ldots, 1)^{T}$. Also, except in part (iii), fix $V_{0}=v$ throughout the proof.

First, recall that any $\mathbb{P}_{x, v}^{\alpha}$ is absolutely continuous with respect to $\widehat{\mathbb{P}}^{\alpha}([10]$, Lemma 6.2), and hence the convergence of $\left\{Z_{n}\right\}$ in law, or the convergence of $\left\{\left|Z_{n}\right|\right\} \widehat{\mathbb{P}}^{\alpha}$-a.s., implies the respective convergence under $\mathbb{P}_{x, v}^{\alpha}$. Thus it is sufficient to prove the convergence results in parts (i) and (ii) with respect to the measure $\widehat{\mathbb{P}}^{\alpha}$, under which the sequence $\left\{\left(M_{n}, Q_{n}\right): n=1,2, \ldots\right\}$ is stationary (cf. Section 2 above), thus allowing us to apply Hennion [26].

(i) Suppose $\mathfrak{m} \in \mathfrak{M}$, and let $x_{\mathfrak{m}}$ be chosen such that $\|\mathfrak{m}\|=\left|\mathfrak{m} x_{\mathfrak{m}}\right|$. Since $\mathfrak{m}$ is nonnegative, an elementary argument shows that $x_{\mathfrak{m}}$ can, in fact, be chosen such that $x_{\mathfrak{m}}^{(i)} \geq 0$ for all $i$. Then for any $x \in \mathbb{S}_{+}^{d-1}$,

$$
|\mathfrak{m} x| \geq\left(\min _{j} x^{(j)}\right)|\mathfrak{m} \overrightarrow{1}| \geq\left(\min _{j} x^{(j)}\right)\left|\mathfrak{m} x_{\mathfrak{m}}\right|=\left(\min _{j} x^{(j)}\right)\|\mathfrak{m}\| .
$$

Thus

$$
\frac{\|\mathfrak{m}\|}{|\mathfrak{m} x|} \leq \frac{1}{\min _{j} x^{(j)}} \quad \text { for all } x \in \mathbb{S}_{+}^{d-1} \text { and all } \mathfrak{m} \in \mathfrak{M}
$$

Recall the stopping time $\mathfrak{N}:=\inf \left\{n \in \mathbb{Z}_{+}: \Pi_{n} \succ 0\right\}$, which is finite $\widehat{\mathbb{P}}^{\alpha}$-a.s. by $\left(\mathrm{H}_{1}\right)$. [Since $\mu_{M}$ is equivalent to $\widehat{\mathbb{P}}^{\alpha}\left(M_{1} \in \cdot\right),\left(\mathrm{H}_{1}\right)$ holds equally well for $\widehat{\mathbb{P}}^{\alpha}\left(M_{1} \in \cdot\right)$. Then Lemma 3.1 of [26] yields finiteness of $\mathfrak{N}$.] Identifying $Q_{0}:=$ $V_{0}=v$ yields

$$
\begin{aligned}
\left|Z_{n}\right| & \leq \sum_{i=0}^{n} \frac{\left|\Pi_{i+1}^{n} Q_{i}\right|}{\left|\Pi_{n} X_{0}\right|} \leq \sum_{i=0}^{n} \frac{\left\|\Pi_{i+1}^{n}\right\|\left|Q_{i}\right|}{\left|\Pi_{i+1}^{n} X_{i}\right|\left|\Pi_{i} X_{0}\right|} \\
& \leq \sum_{i=0}^{\mathfrak{N} \wedge n} \frac{\left\|\Pi_{i+1}^{n}\right\|\left|Q_{i}\right|}{\left|\Pi_{i+1}^{n} X_{i}\right|\left|\Pi_{i} X_{0}\right|}+\sum_{i=\mathfrak{N} \wedge n}^{n} \frac{1}{\min _{j} X_{i}^{(j)}} \frac{\left|Q_{i}\right|}{\left|\Pi_{i} X_{0}\right|} .
\end{aligned}
$$

By [10], Lemma 6.3, $\mathfrak{C}_{i}(x):=\inf _{n \in \mathbb{N}}\left(\left|\Pi_{i+1}^{n} x\right| /\left\|\Pi_{i+1}^{n}\right\|\right)>0 \widehat{\mathbb{P}}^{\alpha}$-a.s., $\forall x \in \mathbb{S}_{+}^{d-1}$. Also $X_{i}^{(j)}=\left(\Pi_{i} X_{0}\right)^{(j)} /\left|\Pi_{i} X_{0}\right|$, implying $X_{i}^{(j)}\left|\Pi_{i} X_{0}\right|=\left(\Pi_{i} X_{0}\right)^{(j)}=\left\langle e_{j}, \Pi_{i} X_{0}\right\rangle$. This identifies the denominator in the second sum of (3.14), and shows that this 
denominator is positive for $i \geq \mathfrak{N}$. Hence

$$
\begin{aligned}
\sup _{n \in \mathbb{N}}\left|Z_{n}\right| & \leq \sum_{i=0}^{\mathfrak{N}} \frac{\left|Q_{i}\right|}{\mathfrak{C}_{i}\left(X_{i}\right)\left|\Pi_{i} X_{0}\right|}+\sum_{i=\mathfrak{N}}^{\infty} \frac{\left|Q_{i}\right|}{\min _{j}\left\langle e_{j}, \Pi_{i} X_{0}\right\rangle} \\
& \leq \sum_{i=0}^{\mathfrak{N}} \frac{\left|Q_{i}\right|}{\mathfrak{C}_{i}\left(X_{i}\right)\left|\Pi_{i} X_{0}\right|}+\sum_{i=\mathfrak{N}}^{\infty} \sum_{j=1}^{d} \frac{\left|Q_{i}\right|}{\left\langle e_{j}, \Pi_{i} X_{0}\right\rangle}
\end{aligned}
$$

Since $\mathfrak{N}<\infty \widehat{\mathbb{P}}^{\alpha}$-a.s., it suffices to focus on the second sum. By Lemma 2.3, we have that $\widehat{\mathbb{P}}^{\alpha}$-a.s.,

$$
\lim _{n \rightarrow \infty} \sup \left\{\left|\frac{1}{n} \mathbf{1}_{\{\mathfrak{N} \leq n\}} \log \left\langle y, \Pi_{n} x\right\rangle-\Lambda^{\prime}(\alpha)\right|: x, y \in \mathbb{S}_{+}^{d-1}\right\}=0 .
$$

Furthermore, by a Borel-Cantelli argument, $\widehat{\mathbb{P}}^{\alpha}\left(\log \left|Q_{i}\right|>\delta i\right.$ i.o. $)=0$, for all $\delta>0$. Thus, given $\varepsilon \in\left(0, \Lambda^{\prime}(\alpha)\right)$, there exists a finite integer $k_{0}$ such that, for all $i \geq k_{0}$ and all $j \in\{1, \ldots, d\}$,

$$
\log \left|Q_{i}\right|-\log \left\langle e_{j}, \Pi_{i} X_{0}\right\rangle \leq-\left(\Lambda^{\prime}(\alpha)-\varepsilon\right) i \quad \widehat{\mathbb{P}}^{\alpha} \text {-a.s. }
$$

Since (3.17) holds uniformly in $j$, substituting (3.17) into (3.15) establishes part (i) of the lemma, where we also use that $\left|Z_{n}^{(0)}\right| \leq\left|Z_{n}\right|$ for all $n \in \mathbb{N}$.

(ii) Following [26], let $\varrho\left(\Pi_{i}^{n}\right)$ denote the spectral radius of $\Pi_{i}^{n}$, and let $R_{n}^{i}$ and $L_{n}^{i}$ denote the right and left eigenvectors corresponding to the maximal eigenvalue in modulus, that is,

$$
\Pi_{i}^{n} R_{n}^{i}=\varrho\left(\Pi_{i}^{n}\right) R_{n}^{i} \quad \text { and } \quad\left(\Pi_{i}^{n}\right)^{T} L_{n}^{i}=\varrho\left(\Pi_{i}^{n}\right) L_{n}^{i}, \quad 1 \leq i \leq n .
$$

Note that the Perron-Frobenius theorem assures that $R_{n}^{i}$ and $L_{n}^{i}$ have nonnegative entries. We further assume the following normalization: $\left|L_{n}^{i}\right|=1,\left\langle L_{n}^{i}, R_{n}^{i}\right\rangle=1$, $1 \leq i \leq n$. Let $\left\{Y_{i}\right\}$ be defined as in (2.8). Then we will show

$$
\lim _{n \rightarrow \infty}\left|\left\langle e_{j}, Z_{n}\right\rangle-\left\langle e_{j}, \widetilde{R}_{n}^{1}\right\rangle \sum_{i=0}^{n} \frac{\left\langle Y_{i}, \widetilde{Q}_{i}\right\rangle}{\left\langle Y_{i}, X_{i}\right\rangle} \frac{\left|Q_{i}\right|}{\left|\Pi_{i} X_{0}\right|}\right|=0 \quad \widehat{\mathbb{P}}^{\alpha} \text {-a.s. }
$$

for all $1 \leq j \leq d$. The sequence $\left\{\widetilde{R}_{n}^{1}\right\}$ converges in distribution as $n \rightarrow \infty$ ([26], Theorem 1(ii)(b)); hence we obtain the convergence, in distribution, of $\left\{Z_{n}\right\}$ to

$$
Z:=\lim _{n \rightarrow \infty} \widetilde{R}_{n}^{1} \cdot \lim _{n \rightarrow \infty} \sum_{i=0}^{n} \frac{\left\langle Y_{i}, \widetilde{Q}_{i}\right\rangle}{\left\langle Y_{i}, X_{i}\right\rangle} \frac{\left|Q_{i}\right|}{\left|\Pi_{i} X_{0}\right|} .
$$

Moreover, since $\left|\widetilde{R}_{n}^{1}\right|=1$, (3.18) yields (3.10), that is, $\lim _{n \rightarrow \infty}\left|Z_{n}\right|=|Z| \widehat{\mathbb{P}}^{\alpha}$-a.s. In the same way, (3.11) is obtained by setting $Q_{0}=0$.

To establish (3.18), first recall (with the identification $Q_{0}:=V_{0}=v$ ) that $Z_{n}=$ $\sum_{i=0}^{n}\left(\Pi_{i+1}^{n} Q_{i} /\left|\Pi_{n} X_{0}\right|\right)$, and observe that

$$
\left|\sum_{i=\lfloor n / 2\rfloor+1}^{n} \frac{\left\langle e_{j}, \Pi_{i+1}^{n} Q_{i}\right\rangle}{\left|\Pi_{n} X_{0}\right|}\right| \leq \sum_{i=\lfloor n / 2\rfloor+1}^{\infty} \frac{\left\|\Pi_{i+1}^{n}\right\|\left|Q_{i}\right|}{\left|\Pi_{i+1}^{n} X_{i}\right|\left|\Pi_{i} X_{0}\right|},
$$


and the right-hand side tends to zero as $n \rightarrow \infty$, by the proof of part (i) [in particular, (3.15)]. Since $Y_{i}$ is a unit vector with nonnegative entries, $\left\langle Y_{i}, X_{i}\right\rangle \geq$ $d^{-1} \min _{j} X_{i}^{(j)}$. Hence we also have

$$
\left|\left\langle e_{j}, \widetilde{R}_{n}^{1}\right\rangle \sum_{i=\lfloor n / 2\rfloor+1}^{n} \frac{\left\langle Y_{i}, \widetilde{Q}_{i}\right\rangle}{\left\langle Y_{i}, X_{i}\right\rangle} \frac{\left|Q_{i}\right|}{\left|\Pi_{i} X_{0}\right|}\right| \leq \sum_{i=\lfloor n / 2\rfloor+1}^{n} \frac{d}{\min _{j} X_{i}^{(j)}} \frac{\left|Q_{i}\right|}{\left|\Pi_{i} X_{0}\right|} .
$$

Thus, to establish (3.18) [and part (ii)], it is enough to show that $\widehat{\mathbb{P}}^{\alpha}$-a.s.,

$$
\lim _{n \rightarrow \infty}\left|\sum_{i=0}^{\lfloor n / 2\rfloor} \frac{\left\langle e_{j}, \Pi_{i+1}^{n} Q_{i}\right\rangle}{\left|\Pi_{n} X_{0}\right|}-\left\langle e_{j}, \widetilde{R}_{n}^{1}\right\rangle \sum_{i=0}^{\lfloor n / 2\rfloor} \frac{\left\langle Y_{i}, \widetilde{Q}_{i}\right\rangle}{\left\langle Y_{i}, X_{i}\right\rangle} \frac{\left|Q_{i}\right|}{\left|\Pi_{i} X_{0}\right|}\right|=0 .
$$

Then by the triangle inequality, it is sufficient to establish the following.

SUBLEMMA 3.7. The following limits hold $\widehat{\mathbb{P}}^{\alpha}-$ a.s.:

$$
\begin{aligned}
& \lim _{n \rightarrow \infty} \sum_{i=0}^{\lfloor n / 2\rfloor}\left|\frac{\left\langle e_{j}, \Pi_{i+1}^{n} Q_{i}\right\rangle}{\left|\Pi_{n} X_{0}\right|}-\left\langle e_{j}, \widetilde{R}_{n}^{i+1}\right\rangle \frac{\left\langle L_{n}^{i+1}, Q_{i}\right\rangle}{\left\langle L_{n}^{i+1}, X_{i}\right\rangle} \frac{1}{\left|\Pi_{i} X_{0}\right|}\right|=0 ; \\
& \lim _{n \rightarrow \infty} \sum_{i=0}^{\lfloor n / 2\rfloor}\left|\frac{\left\langle e_{j}, \widetilde{R}_{n}^{i+1}\right\rangle}{\left|\Pi_{i} X_{0}\right|}\left(\frac{\left\langle L_{n}^{i+1}, Q_{i}\right\rangle}{\left\langle L_{n}^{i+1}, X_{i}\right\rangle}-\frac{\left\langle Y_{i+1}, Q_{i}\right\rangle}{\left\langle Y_{i+1}, X_{i}\right\rangle}\right)\right|=0 ; \\
& \lim _{n \rightarrow \infty} \sum_{i=0}^{\lfloor n / 2\rfloor}\left|\frac{1}{\left|\Pi_{i} X_{0}\right|} \frac{\left\langle Y_{i+1}, Q_{i}\right\rangle}{\left\langle Y_{i+1}, X_{i}\right\rangle}\left(\left\langle e_{j}, \widetilde{R}_{n}^{i+1}\right\rangle-\left\langle e_{j}, \widetilde{R}_{n}^{1}\right\rangle\right)\right|=0 .
\end{aligned}
$$

Proof of the Sublemma. For (3.20), observe by [26], Corollary 1, that

$$
\lim _{n \rightarrow \infty}\left(\frac{\Pi_{i+1}^{n}}{\left\|\Pi_{i+1}^{n}\right\|}-\frac{R_{n}^{i+1} \otimes L_{n}^{i+1}}{\left\|R_{n}^{i+1} \otimes L_{n}^{i+1}\right\|}\right)=0 \quad \widehat{\mathbb{P}}^{\alpha} \text {-a.s. }
$$

where $a \otimes b$ is the rank-one matrix with $\left\langle e_{i},(a \otimes b) e_{j}\right\rangle=\left\langle e_{i}, a\right\rangle\left\langle b, e_{j}\right\rangle$. From (3.23), we infer the asymptotic identities

$$
\begin{aligned}
\lim _{n \rightarrow \infty}\left(\frac{\left\langle e_{j}, \Pi_{i+1}^{n} Q_{i}\right\rangle}{\left\|\Pi_{i+1}^{n}\right\|}-\frac{\left\langle e_{j}, R_{n}^{i+1}\right\rangle\left\langle L_{n}^{i+1}, Q_{i}\right\rangle}{\left\|R_{n}^{i+1} \otimes L_{n}^{i+1}\right\|}\right) & =0 \\
\lim _{n \rightarrow \infty}\left(\frac{\left|\Pi_{i+1}^{n} X_{i}\right|}{\left\|\Pi_{i+1}^{n}\right\|}-\frac{\left|R_{n}^{i+1}\right|\left\langle L_{n}^{i+1}, X_{i}\right\rangle}{\left\|R_{n}^{i+1} \otimes L_{n}^{i+1}\right\|}\right) & =0 .
\end{aligned}
$$

Combining (3.24) and (3.25), we conclude that

$$
\begin{aligned}
\lim _{n \rightarrow \infty} \frac{\left\langle e_{j}, \Pi_{i+1}^{n} Q_{i}\right\rangle}{\left|\Pi_{n} X_{0}\right|} & =\lim _{n \rightarrow \infty} \frac{\left\langle e_{j}, \Pi_{i+1}^{n} Q_{i}\right\rangle}{\left|\Pi_{i+1}^{n} X_{i}\right|\left|\Pi_{i} X_{0}\right|} \\
& =\lim _{n \rightarrow \infty}\left\langle e_{j}, \widetilde{R}_{n}^{i+1}\right\rangle \frac{\left\langle L_{n}^{i+1}, Q_{i}\right\rangle}{\left\langle L_{n}^{i+1}, X_{i}\right\rangle} \frac{1}{\left|\Pi_{i} X_{0}\right|} \quad \widehat{\mathbb{P}}^{\alpha} \text {-a.s., }
\end{aligned}
$$

showing, in particular, that the individual terms in $(3.20) \rightarrow 0 \widehat{\mathbb{P}}^{\alpha}$-a.s. 
To prove that the sum in (3.20) converges to zero, we now invoke a dominated convergence argument. Since $\mathfrak{N}$ is finite a.s., it suffices to focus on summands with $i \geq \mathfrak{N}$, where we can assume that all components of $X_{i}$ are positive, as the remaining terms form a finite sum. Observe that

$$
\frac{\left\langle L_{n}^{i+1}, \overrightarrow{1}\right\rangle \max _{j} Q_{i}^{(j)}}{\left\langle L_{n}^{i+1}, \overrightarrow{1}\right\rangle \min _{j} X_{i}^{(j)}} \leq \frac{\left|Q_{i}\right|}{\min _{j} X_{i}^{(j)}}
$$

and, therefore,

$$
\begin{gathered}
\sup _{n} \sum_{i=\mathfrak{N}}^{\lfloor n / 2\rfloor}\left|\frac{\left\langle e_{j}, \Pi_{i+1}^{n} Q_{i}\right\rangle}{\left|\Pi_{n} X_{0}\right|}-\left\langle e_{j}, \widetilde{R}_{n}^{i+1}\right\rangle \frac{\left\langle L_{n}^{i+1}, Q_{i}\right\rangle}{\left\langle L_{n}^{i+1}, X_{i}\right\rangle} \frac{1}{\left|\Pi_{i} X_{0}\right|}\right| \\
\leq 2 \sup _{n} \sum_{i=\mathfrak{N}}^{\lfloor n / 2\rfloor} \frac{1}{\min _{i} X_{i}^{(j)}} \frac{\left|Q_{i}\right|}{\left|\Pi_{i} X_{0}\right|}<\infty \quad \widehat{\mathbb{P}}^{\alpha} \text {-a.s., }
\end{gathered}
$$

by part (i) [where we have used the calculation in (3.14) to handle the first term on the left-hand side]. Thus, using a dominated convergence argument [applied pointwise on the space where (3.25) and (3.28) hold], we deduce that (3.20) follows from (3.25).

Next, we turn to (3.21). It follows by Lemma 3.3 of [26] that, under $\widehat{\mathbb{P}}^{\alpha}$, the sequence $\left\{L_{n}^{i+1}\right\}$ converges a.s. as $n \rightarrow \infty$ to $Y_{i+1}$. Hence, by a dominated convergence argument, we conclude that (3.21) holds.

Finally, to establish (3.22), note by Proposition 3.1 of [26] that

$$
\left|\widetilde{R}_{n}^{i+1}-\widetilde{R}_{n}^{1}\right|=\left|\left(\Pi_{i+1}^{n} R_{n}^{i+1}\right)^{\sim}-\left(\Pi_{n} R_{n}^{1}\right)^{\sim}\right| \leq 2 \mathbf{c}\left(\Pi_{i+1}^{n}\right),
$$

where $\mathbf{c}(\cdot)$ is bounded above by one and tends to zero $\widehat{\mathbb{P}}^{\alpha}$-a.s. as $(n-i) \rightarrow \infty$ ([26], Lemma 3.2). Then (3.22) follows, once again, by the dominated convergence theorem. This completes the proof of the sublemma and, consequently, part (ii) of Lemma 3.6.

PROOF OF LEMma 3.6 (CONTINUED). We now return to the proof of main lemma, where it remains to verify that (iii) and (iv) hold.

(iii) Let $m \in \mathbb{N}$ and $B_{1}=\max _{x, y}\left(r_{\alpha}(x) / r_{\alpha}(y)\right) \in(0, \infty)$. Then for $\alpha>0$,

$$
\begin{aligned}
\mathbb{E}_{\boldsymbol{\delta}_{v}}^{\alpha}[ & \left.\left(\sup _{n \leq m}\left|Z_{n}\right| \mathbf{1}_{\left\{\tau^{\prime} \geq n\right\}}\right)^{\alpha}\right] \\
& \leq \mathbb{E}_{\boldsymbol{\delta}_{v}}^{\alpha}\left[\left(\sup _{n \leq m}\left(|v|+\sum_{k=1}^{n} \frac{\left|\Pi_{k+1}^{n} Q_{k}\right|}{\left|\Pi_{n} X_{0}\right|} \mathbf{1}_{\left\{\tau^{\prime} \geq k-1\right\}}\right)\right)^{\alpha}\right] \\
& =\left(r_{\alpha}(\widetilde{v})\right)^{-1} \mathbb{E}_{\delta_{v}}\left[r_{\alpha}\left(X_{m}\right)\left|\Pi_{m} X_{0}\right|^{\alpha}\left(\sup _{n \leq m}\left(|v|+\sum_{k=1}^{n} \frac{\left|\Pi_{k+1}^{n} Q_{k}\right|}{\left|\Pi_{n} X_{0}\right|} \mathbf{1}_{\left\{\tau^{\prime} \geq k-1\right\}}\right)\right)^{\alpha}\right]
\end{aligned}
$$




$$
\begin{aligned}
& \leq B_{1} \mathbb{E}_{\boldsymbol{\delta}_{v}}\left[\left(\sup _{n \leq m}\left(\left|\Pi_{m} X_{0}\right||v|+\sum_{k=1}^{n}\left|\Pi_{n+1}^{m} X_{n}\right| \cdot\left|\Pi_{k+1}^{n} Q_{k}\right| \mathbf{1}_{\left\{\tau^{\prime} \geq k-1\right\}}\right)\right)^{\alpha}\right] \\
& \leq B_{1} \mathbb{E}_{v}\left[\sup _{n \leq m}\left(\sum_{k=0}^{m}\left\|\Pi_{n+1}^{m}\right\| \cdot\left\|\Pi_{k+1}^{n}\right\| \cdot\left|Q_{k}\right| \mathbf{1}_{\left\{\tau^{\prime} \geq k-1\right\}}\right)^{\alpha}\right] \quad \text { where } Q_{0}:=v .
\end{aligned}
$$

Now suppose that $\alpha \geq 1$. Then by Minkowski's inequality,

$$
\begin{aligned}
\left(\mathbb{E}_{v}[\right. & \left.\left.\left(\sum_{k=0}^{m}\left\|\Pi_{n+1}^{m}\right\| \cdot\left\|\Pi_{k+1}^{n}\right\| \cdot\left|Q_{k}\right| \mathbf{1}_{\left\{\tau^{\prime} \geq k-1\right\}}\right)^{\alpha}\right]\right)^{1 / \alpha} \\
& \leq \sum_{k=0}^{m}\left(\mathbb{E}\left[\left\|\Pi_{n+1}^{m}\right\|^{\alpha}\right]\right)^{1 / \alpha}\left(\mathbb{E}\left[\left\|\Pi_{k+1}^{n}\right\|^{\alpha}\right]\right)^{1 / \alpha}\left(|v|+\mathbb{E}\left[\left|Q_{1}\right|^{\alpha}\right]\right)^{1 / \alpha} p_{k}^{1 / \alpha},
\end{aligned}
$$

where $p_{k}:=\mathbb{P}_{v}\left(\tau^{\prime} \geq k-1\right)$. Now by [10], Corollary 4.6, $\mathbb{E}\left[\left\|\Pi_{n}\right\|^{\alpha}\right] \leq B_{2} \in$ $(0, \infty)$, for all $n$. Moreover, $\mathbb{E}\left[\left|Q_{i}\right|^{\alpha}\right]<\infty$ by $\left(\mathrm{H}_{2}\right)$; and by the assumption of part (iii), $p_{k} \leq B_{3} t^{k}$ for some $t \in(0,1)$ (uniformly in $v$ ). Combining these estimates yields

$$
\mathbb{E}_{\boldsymbol{\delta}_{v}}^{\alpha}\left[\sup _{n \in \mathbb{N}}\left|Z_{n}\right|^{\alpha} \mathbf{1}_{\left\{\tau^{\prime} \geq n\right\}}\right] \leq B_{1} B_{2}^{2}\left(|v|+\mathbb{E}\left[\left|Q_{1}\right|^{\alpha}\right]\right)\left(\sum_{k=0}^{\infty}\left(B_{3} t^{k}\right)^{1 / \alpha}\right)^{\alpha}<\infty,
$$

and this bound is uniform over $v \in F$, for any bounded set $F \subset \mathbb{R}_{+}^{d} \backslash\{0\}$.

If $\alpha \leq 1$, then we use the subadditivity, namely the inequality $|x+y|^{\alpha} \leq|x|^{\alpha}+$ $|y|^{\alpha}$ in place of Minkowski's inequality, and then proceed as before.

Now it follows from part (ii) that $\left|Z_{n}\right|^{\alpha} \mathbf{1}_{\left\{\tau^{\prime} \geq n\right\}} \rightarrow|Z|^{\alpha} \mathbf{1}_{\left\{\tau^{\prime}=\infty\right\}} \mathbb{P}^{\alpha}$-a.s. as $n \rightarrow \infty$. Consequently,

$$
\sup _{v \in F} \mathbb{E}_{\boldsymbol{\delta}_{v}}^{\alpha}\left[|Z|^{\alpha} \mathbf{1}_{\left\{\tau^{\prime}=\infty\right\}}\right] \leq \sup _{v \in F} \mathbb{E}_{\boldsymbol{\delta}_{v}}^{\alpha}\left[\sup _{n \in \mathbb{N}}\left|Z_{n}\right|^{\alpha} \mathbf{1}_{\left\{\tau^{\prime} \geq n\right\}}\right]<\infty .
$$

(iv) The almost sure convergence $\left|Z_{n}\right|^{\alpha} \mathbf{1}_{\left\{\tau^{\prime} \geq n\right\}} \rightarrow|Z|^{\alpha} \mathbf{1}_{\left\{\tau^{\prime}=\infty\right\}}$ was obtained in part (ii), and it was shown in part (iii) that $\left\{\left|Z_{n}\right|^{\alpha} \mathbf{1}_{\left\{\tau^{\prime} \geq n\right\}}\right\}_{n \in \mathbb{N}}$ is uniformly integrable, and the $L^{1}$-convergence follows.

3.3. Markov nonlinear renewal theory. Set $\mathfrak{T}_{u}=\inf \left\{n \in \mathbb{N}_{+}: S_{n}>\log u\right\}$. Assuming $\left(\mathrm{H}_{1}\right)$ and $\left(\mathrm{H}_{2}\right)$, then Kesten [28], Theorem 2, proved that there is a probability measure $\varrho$ on $\mathbb{S}_{+}^{d-1} \times(0, \infty)$, namely the asymptotic overjump distribution, for which we have the weak convergence

$$
\left(X_{\mathfrak{T}_{u}}, S_{\mathfrak{T}_{u}}-\log u\right) \Rightarrow \varrho(\cdot) \quad \text { as } u \rightarrow \infty .
$$

If the function $d_{A}$ is bounded and continuous, and we define $S_{n}^{A}:=S_{n}-$ $\log d_{A}\left(X_{n}\right)$ and $\mathfrak{T}_{u}^{A}=\inf \left\{n \in \mathbb{N}_{+}: S_{n}^{A}>\log u\right\}$, then only minor modifications are needed to deduce that for a certain probability measure $\varrho^{A}$,

$$
\left(X_{\mathfrak{T}_{u}}, S_{\mathfrak{T}_{u}}^{A}-\log u\right) \Rightarrow \varrho^{A}(\cdot) \quad \text { as } u \rightarrow \infty .
$$


In this section, we apply the Markov nonlinear renewal theory developed by Melfi $[34,35]$ to obtain the asymptotic overjump distributions for the processes $\left\{V_{n}\right\}$ and $\left\{V_{n}^{A}\right\}$, where

$$
V_{n}^{A}:=\frac{V_{n}}{d_{A}\left(\widetilde{V}_{n}\right)}, \quad n \in \mathbb{N} .
$$

First, recall the definitions of $T_{u}, T_{u}^{A}$ in (2.16), (2.17) and note that it follows from the definitions that $T_{u}^{A}=\inf \left\{n \in \mathbb{N}_{+}:\left|V_{n}^{A}\right|>u\right\}$.

THEOREM 3.8. Assume $\left(\mathrm{H}_{1}\right)$ and $\left(\mathrm{H}_{2}\right)$. Let $d_{A} \in \mathscr{C}_{b}\left(\mathbb{S}_{+}^{d-1}\right)$. Then for all $f \in$ $\mathscr{C}_{b}\left(\mathbb{S}_{+}^{d-1} \times(0, \infty)\right)$ and all $x \in \mathbb{S}_{+}^{d-1}$ and $v \in \mathbb{R}_{+}^{d} \backslash\{0\}$,

$$
\lim _{u \rightarrow \infty} \mathbb{E}_{x, v}^{\alpha}\left[f\left(\tilde{V}_{T_{u}}, \log \frac{\left|V_{T_{u}}\right|}{u}\right)\right]=\int_{\mathbb{S}_{+}^{d-1} \times \mathbb{R}_{+}} f(y, s) \varrho(d y, d s),
$$

where $\varrho$ is given as in (3.30). Moreover, the same result also holds if $\left(\varrho, V_{T_{u}}\right)$ is replaced with $\left(\varrho^{A}, V_{T_{u}^{A}}^{A}\right)$.

ProOF. We need to verify conditions (I'), (II) and (III) of [35], Theorem 3, for the process $\left\{\left(W_{\log u}, Z_{\log u}, R_{\log u}\right)=\left(\widetilde{V}_{T_{u}}, \log \left|V_{T_{u}}\right|, \log \left|V_{T_{u}}\right|-\log u\right)\right\}$.

Condition (III), namely tightness of $\left\{\widetilde{V}_{T_{u}}\right\}$, is satisfied since $\mathbb{S}_{+}^{d-1}$ is compact. The validity of Conditions (I) and (II) is proved below in Lemmas 3.9 and 3.11, respectively. Then (3.33) follows from [35], Theorem 3.

Turning to the case where $\left(\varrho, \widetilde{V}_{T_{u}}\right)$ is replaced with $\left(\varrho^{A}, \widetilde{V}_{T_{u}^{A}}^{A}\right)$, we need to check the validity of Conditions (I') and (II) for $\left\{\left(\widetilde{V}_{n}^{A}, \log \left|V_{n}^{A}\right|\right)\right\}$. By (3.32), $\widetilde{V}_{n}^{A}=\widetilde{V}_{n}$. Thus, for $f(x, s)=\left(x, s-\log d_{A}(x)\right)$, we have that $\left\{\left(\widetilde{V}_{n}^{A}, \log \left|V_{n}^{A}\right|\right)\right\}=$ $\left\{f\left(\tilde{V}_{n}, \log \left|V_{n}\right|\right)\right\}$ and $\left\{\left(X_{n}, S_{n}^{A}\right)\right\}=\left\{f\left(X_{n}, S_{n}\right)\right\}$ [using (2.18)]. Hence $\left(\mathrm{I}^{\prime}\right)$ can be deduced from Lemma 3.9 below. Finally, since $d_{A}$ is bounded, the tightness of $\left\{\log \left|V_{T_{u}^{A}}^{A}\right|-\log u\right\}=\left\{\log \left|V_{T_{u}^{A}}\right|-\log d_{A}\left(V_{T_{u}^{A}}\right)-\log u\right\}$ follows from Lemma 3.11.

Write $\mathfrak{d}_{m}$ for the Prokhorov distance on the space of probability measures on $\left(\mathbb{S}_{+}^{d-1} \times[0, \infty)\right)^{m}$.

Lemma 3.9. Assume $\left(\mathrm{H}_{1}\right)$ and $\left(\mathrm{H}_{2}\right)$. Then for all $m \in \mathbb{N}_{+}$,

$$
\mathfrak{d}_{m}\left(\mathbb{P}^{\alpha}\left(\left(\tilde{V}_{T_{u}+k}, \log \frac{\left|V_{T_{u}+k}\right|}{\left|V_{T_{u}}\right|}\right)_{k=1}^{m} \in \cdot \mid \mathscr{F}_{T_{u}}\right), \mathbb{P}_{Y_{T_{u}}}^{\alpha}\left(\left(X_{k}, S_{k}\right)_{k=1}^{m} \in \cdot\right)\right)
$$

converges to zero as $u \rightarrow \infty$ in $\mathbb{P}^{\alpha}$-probability.

PROOF. Using the Markov property,

$$
\begin{array}{r}
\mathbb{P}^{\alpha}\left(\left(\widetilde{V}_{T_{u}+k}, \log \left|V_{T_{u}+k}\right|-\log \left|V_{T_{u}}\right|\right)_{1 \leq k \leq m} \in \cdot \mid \mathscr{F}_{T_{u}}\right) \\
\quad=\mathbb{P}_{X_{T_{u}}, V_{T_{u}}}^{\alpha}\left(\left(\widetilde{V}_{k}, \log \left|V_{k}\right|-\log \left|V_{0}\right|\right)_{1 \leq k \leq m} \in \cdot\right) .
\end{array}
$$


By [25], Lemma 3.5, the total variation distance between $\mathbb{P}_{x, v}^{\alpha}$ and $\mathbb{P}_{y, v}^{\alpha}$ is bounded above by $B|x-y|^{\bar{\alpha}}$ for some $B<\infty$, where $\bar{\alpha}=\min \{\alpha, 1\}$. (The proof in [25] is for invertible matrices, but carries over to nonnegative matrices.) Then, as total variation distance is an upper bound for the Prokhorov distance,

$$
\begin{aligned}
\mathfrak{d}_{m}\left(\mathbb{P}_{X_{T_{u}}, V_{T_{u}}}^{\alpha}\left(\left(\widetilde{V}_{k}, \log \left|V_{k}\right|-\log \left|V_{0}\right|\right)_{k=1}^{m} \in \cdot\right), \mathbb{P}_{\widetilde{V}_{T_{u}}}^{\alpha}\left(\left(X_{k}, S_{k}\right)_{k=1}^{m} \in \cdot\right)\right) \\
\quad \leq B\left|X_{T_{u}}-\widetilde{V}_{T_{u}}\right|^{\bar{\alpha}} \\
\quad+\mathfrak{d}_{m}\left(\mathbb{P}_{\widetilde{V}_{T_{u}}, V_{T_{u}}}^{\alpha}\left(\left(\widetilde{V}_{k}, \log \left|V_{k}\right|-\log \left|V_{0}\right|\right)_{k=1}^{m} \in \cdot\right), \mathbb{P}_{\widetilde{V}_{T_{u}}}^{\alpha}\left(\left(X_{k}, S_{k}\right)_{k=1}^{m} \in \cdot\right)\right) .
\end{aligned}
$$

It will be proved in Lemma 3.10 below that $\left|X_{T_{u}}-\widetilde{V}_{T_{u}}\right|$ tends to zero in $\mathbb{P}^{\alpha}$ probability. We thus consider only the last term. Fix the initial values $\left(\widetilde{V}_{T_{u}}, V_{T_{u}}\right)=$ $(\tilde{v}, v)$, and introduce the notation $V_{1}^{(0)}=Q_{1}$ and $V_{k}^{(0)}:=\sum_{j=1}^{k} M_{k} \cdots M_{j+1} Q_{j}$. Then standard estimates yield, for all $v \in \mathbb{R}_{+}^{d} \backslash\{0\}$,

$$
\begin{gathered}
\mathbb{P}_{\tilde{v}}^{\alpha}\left(\left|\left(\widetilde{V}_{k}, \log \left|V_{k}\right|-\log \left|V_{0}\right|\right)_{1 \leq k \leq m}-\left(X_{k}, S_{k}\right)_{1 \leq k \leq m}\right|_{\infty} \geq \varepsilon \mid V_{0}=v\right) \\
\leq \mathbb{P}_{\tilde{v}}^{\alpha}\left(2 \sum_{k=1}^{m} \frac{\left|V_{k}^{(0)}\right|}{\left|\Pi_{k} v\right|} \geq \varepsilon\right) \leq \frac{B}{\varepsilon^{\alpha}|v|^{\alpha}} \sum_{k=1}^{m} \mathbb{E}\left[\left|V_{k}^{(0)}\right|^{\alpha}\right]
\end{gathered}
$$

for some universal constant $B$, where we used Chebyshev's inequality and boundedness of $r_{\alpha}$ in the last inequality. Hence

$$
\lim _{u \rightarrow \infty} \sup _{v:|v| \geq u} \mathbb{P}_{\tilde{v}}^{\alpha}\left(\left|\left(\tilde{V}_{k}, \log \frac{\left|V_{k}\right|}{\left|V_{0}\right|}\right)_{k=1}^{m}-\left(X_{k}, S_{k}\right)_{k=1}^{m}\right|_{\infty} \geq \varepsilon \mid V_{0}=v\right)=0 .
$$

Recall that convergence in probability implies convergence in the Prokhorov metric. Since $\mathbb{P}^{\alpha}\left(T_{u}<\infty\right)=1$ and $\left|V_{T_{u}}\right| \geq u$, we infer the $\mathbb{P}^{\alpha}$-a.s. convergence, namely, as $u \rightarrow \infty$,

$$
\mathfrak{d}_{m}\left(\mathbb{P}_{\widetilde{V}_{T_{u}}, V_{T_{u}}}^{\alpha}\left(\left(\widetilde{V}_{k}, \log \frac{\left|V_{k}\right|}{\left|V_{0}\right|}\right)_{k=1}^{m} \in \cdot\right), \mathbb{P}_{\widetilde{V}_{T_{u}}}^{\alpha}\left(\left(X_{k}, S_{k}\right)_{k=1}^{m} \in \cdot\right)\right) \rightarrow 0 .
$$

LEMMA 3.10. For all $x \in \mathbb{S}_{+}^{d-1}$ and $V_{0}=v \in \mathbb{R}_{+}^{d} \backslash\{0\}$,

$$
\lim _{u \rightarrow \infty}\left|X_{T_{u}}-\widetilde{V}_{T_{u}}\right|=0 \quad \text { in } \mathbb{P}_{x, v}^{\alpha} \text {-probability. }
$$

PROOF. Let $w=u / 2$, and decompose the process based on its behavior prior and subsequent to the time $T_{w}$. Recalling that $\Pi_{i}^{n}:=M_{n} \cdots M_{i}$ and using the triangle inequality, we see that $\mathbb{P}_{x}^{\alpha}\left(\left|\widetilde{V}_{T_{u}}-X_{T_{u}}\right|>\varepsilon\right)$ can be written as

$$
\begin{aligned}
& \mathbb{P}_{x}^{\alpha}\left(\left|\frac{\Pi_{T_{w}+1}^{T_{u}} V_{T_{w}}+\sum_{i=T_{w+1}}^{T_{u}} \Pi_{i+1}^{T_{u}} Q_{i}}{\left|V_{T_{u}}\right|}-\left(\Pi_{T_{w}+1}^{T_{u}} X_{T_{w}}\right)^{\sim}\right|>\varepsilon\right) \\
& \quad \leq \mathbb{E}_{x}^{\alpha}\left[\mathbb{P}_{x}^{\alpha}\left(\left|\frac{\Pi_{T_{w}+1}^{T_{u}} V_{T_{w}}+\sum_{i=T_{w}+1}^{T_{u}} \Pi_{i+1}^{T_{u}} Q_{i}}{\left|V_{T_{u}}\right|}-\frac{\Pi_{T_{w}+1}^{T_{u}} V_{T_{w}}}{\left|\Pi_{T_{w}+1}^{T_{u}} V_{T_{w}}\right|}\right|>\frac{\varepsilon}{2} \mid \mathscr{F}_{T_{w}}\right)\right]
\end{aligned}
$$




$$
\begin{aligned}
& +\mathbb{P}_{x}^{\alpha}\left(\left|\left(\Pi_{T_{w}+1}^{T_{u}} V_{T_{w}}\right)^{\sim}-\left(\Pi_{T_{w}+1}^{T_{u}} X_{T_{w}}\right)^{\sim}\right|>\frac{\varepsilon}{2}\right) \\
:= & \mathbb{I}_{1}(u)+\mathbb{I}_{2}(u) .
\end{aligned}
$$

To compute $\mathbb{I}_{2}(u)$ as $u \rightarrow \infty$, we apply Proposition 3.1 of [26], which yields

$$
\sup _{x, y \in \mathbb{S}_{+}^{d-1}}\left|\left(\Pi_{i+1}^{n} x\right)^{\sim}-\left(\Pi_{i+1}^{n} y\right)^{\sim}\right| \leq 2 \mathbf{c}\left(\Pi_{i+1}^{n}\right)
$$

for a function $\mathbf{c}(\cdot)$ which is bounded above by one and tends to zero $\widehat{\mathbb{P}}^{\alpha}$-a.s. as $(n-i) \rightarrow \infty$ ([26], Lemma 3.2). Since $\mathbb{P}_{x}^{\alpha}$ is absolutely continuous with respect to the measure $\widehat{\mathbb{P}}^{\alpha}\left([10]\right.$, Lemma 6.2), it follows that $\mathbf{c}\left(\Pi_{T_{u / 2}+1}^{T_{u}}\right) \rightarrow 0 \mathbb{P}_{x}^{\alpha}$-a.s. for all $x \in \mathbb{S}_{+}^{d-1}$, and hence

$$
\mathbb{I}_{2}(u) \leq \mathbb{P}_{x}^{\alpha}\left(2 \mathbf{c}\left(\Pi_{T_{w}+1}^{T_{u}}\right)>\frac{\varepsilon}{2}\right) \searrow 0 \quad \text { as } u \rightarrow \infty .
$$

Now consider $\mathbb{I}_{1}(u)$ as $u \rightarrow \infty$. Standard estimates yield

$$
\mathbb{I}_{1}(u) \leq \mathbb{E}_{x}^{\alpha}\left[\mathbb{P}_{\widetilde{V}_{T_{w}}^{\alpha}}^{\alpha}\left(\frac{4\left|V_{T_{u}}^{(0)}\right|}{u\left|\Pi_{T_{u}} \widetilde{V}_{0}\right|}>\frac{\varepsilon}{2}\right)\right]
$$

Next, recall by Lemma 3.6(i) that $\mathscr{Z}^{0}:=\sup _{n \in \mathbb{N}}\left(\left|V_{n}^{(0)}\right| /\left|\Pi_{n} X_{0}\right|\right)<\infty \widehat{\mathbb{P}}^{\alpha}$-a.s., and this quantity does not depend on the initial value, $V_{0}$. Using that $\mathbb{P}_{\tilde{V}_{T_{w}}^{\alpha}}^{\alpha}(\cdot) \leq$ $B \widehat{\mathbb{P}}^{\alpha}(\cdot)$ for some universal constant $B([10]$, Lemma 6.2), we then obtain that

$$
\mathbb{I}_{1}(u) \leq B \lim _{u \rightarrow \infty} \widehat{\mathbb{P}}^{\alpha}\left(\frac{\mathscr{Z}^{0}}{u}>\frac{\varepsilon}{8}\right)=0
$$

The following lemma concludes the proof of Theorem 3.8.

LEMMA 3.11. $\left\{\widetilde{V}_{T_{u}}-\log u\right\}_{u \geq 1}$ is tight under $\mathbb{P}^{\alpha}$.

PROOF. A sufficient condition is given in [35], Section 5.2: Letting $\xi_{n}:=$ $\log \left|V_{n}\right|-S_{n}$ and supposing that $\left\{\xi_{T_{u}}\right\}_{u \geq 1}$ and $\left\{\xi_{\mathfrak{T}_{u}}\right\}_{u \geq 1}$ are tight under $\mathbb{P}^{\alpha}$, then it follows that $\left\{W_{T_{u}}-\log u\right\}_{u \geq 1}$ is tight.

Now by Lemma 3.6,

$$
\xi_{n}=\log \frac{\left|V_{n}\right|}{\left|\Pi_{n} V_{0}\right|} \rightarrow \log Z \quad \mathbb{P}^{\alpha} \text {-a.s. }
$$

for a finite random variable $Z$. Since $T_{u}$ and $\mathfrak{T}_{u}$ are stopping times with respect to the filtration $\left\{\mathscr{F}_{n}\right\}$ and tend to infinity as $u \rightarrow \infty$, we deduce that

$$
\lim _{u \rightarrow \infty} \xi_{T_{u}}=\log Z \quad \mathbb{P}^{\alpha} \text {-a.s. and } \quad \lim _{u \rightarrow \infty} \xi_{\mathfrak{T}_{u}}=\log Z \quad \mathbb{P}^{\alpha} \text {-a.s. }
$$

Thus, in particular, the families $\left\{\xi_{T_{u}}\right\}_{u \geq 1}$ and $\left\{\xi_{\mathfrak{T}_{u}}\right\}_{u \geq 1}$ converge in distribution under $\mathbb{P}^{\alpha}$ and are consequently tight.

The last result concerns the first passage times in the $\alpha$-shifted measure. 
LEMMA 3.12. Assume that $d_{A}$ is bounded and continuous. Then

$$
\lim _{u \rightarrow \infty} \frac{T_{u}^{A}}{\log u}=\frac{1}{\lambda^{\prime}(\alpha)} \quad \text { in } \mathbb{P}^{\alpha} \text {-probability. }
$$

PROOF. By definition, $V_{n}=Z_{n} e^{S_{n}}$ and $V_{n}^{A}=V_{n} / d_{A}\left(\tilde{V}_{n}\right)$, and consequently

$$
\log \left|V_{n}^{A}\right|=S_{n}+\left|Z_{n}\right|-\log d_{A}\left(\widetilde{V}_{n}\right):=S_{n}+\xi_{n} .
$$

Recall that $\sup _{n \in \mathbb{N}} Z_{n}$ is finite a.s., by Lemma 3.6. Since $d_{A}$ is bounded, it follows that the sequence $\left\{\xi_{i}\right\}$ in (3.39) is slowly changing [as defined in [46], equation (9.5)]. Now by Lemma 2.3, $S_{n} / n \rightarrow \lambda^{\prime}(\alpha)$ a.s., and hence $\log \left|V_{n}^{A}\right| / n \rightarrow \lambda^{\prime}(\alpha)$ a.s. The result then follows by reasoning as in [46], Lemma 9.13.

\section{Characterizing the large exceedances over cycles.}

4.1. Proposition 4.1 and its consequences. Recall that $\tau$ denotes the return time to a set $\mathbb{D}=B_{r}^{+}(0)$, where $\pi(\mathbb{D})>0$, and $T_{u}^{A}:=\inf \left\{n: V_{n} \in u A\right\}=$ $\inf \left\{n:\left|V_{n}^{A}\right|>u\right\}$, where $V_{n}^{A}:=V_{n} / d_{A}\left(\widetilde{V}_{n}\right)$ [cf. (3.32)]. Also recall that $r_{\alpha}^{A}(x):=$ $r_{\alpha}(x)\left(d_{A}(x)\right)^{\alpha}, x \in \mathbb{S}_{+}^{d-1}$.

We say that a function $g:\left(\mathbb{R}_{+}^{d}\right)^{m+1} \rightarrow \mathbb{R}$ is almost $\theta$-Hölder continuous if

$$
g\left(v_{0}, \ldots, v_{m}\right)=\hat{g}\left(v_{0}, \ldots, v_{m}\right) \mathbf{1}_{\left\{\left|v_{m}\right| \geq \delta\right\}}
$$

for some $\delta \geq 0$ and $\theta$-Hölder continuous function $\hat{g}$.

Proposition 4.1. Assume $\left(\mathrm{H}_{1}\right)$ and $\left(\mathrm{H}_{2}\right)$ are satisfied. Let $m \in \mathbb{N}$ and $g:\left(\mathbb{R}_{+}^{d}\right)^{m+1} \rightarrow \mathbb{R}$ be a bounded almost $\theta$-Hölder continuous function for $\theta \leq$ $\min \{1, \alpha\}$, and assume that the function $d_{A}$ is bounded and continuous on $\mathbb{S}_{+}^{d-1}$. Then for any $v \in \mathbb{R}_{+}^{d} \backslash\{0\}$,

$$
\begin{aligned}
\lim _{u \rightarrow \infty} u^{\alpha} & \mathbb{E}\left[g\left(\frac{V_{T_{u}^{A}}}{u}, \ldots, \frac{V_{T_{u}^{A}+m}}{u}\right) \mathbf{1}_{\left\{T_{u}^{A}<\tau\right\}} \mid V_{0}=v\right] \\
= & r_{\alpha}(\widetilde{v}) \mathbb{E}_{\boldsymbol{\delta}_{v}}^{\alpha}\left[|Z|^{\alpha} \mathbf{1}_{\{\tau=\infty\}}\right] \\
& \times \int \frac{e^{-\alpha s}}{r_{\alpha}^{A}(x)} \mathbb{E}\left[g\left(e^{S_{0}} X_{0}, \ldots, e^{S_{m}} X_{m}\right) \mid X_{0}=x, S_{0}=s+\log d_{A}(x)\right] \\
& \times \varrho^{A}(d x, d s) .
\end{aligned}
$$

Recall that $\varrho^{A}$ is the asymptotic overjump distribution related to $\left\{V_{T_{u}^{A}}^{A}\right\}$, while on the left-hand side of the above equation, we evaluate $g$ for the process $\left\{V_{n}\right\}$ (not $\left\{V_{n}^{A}\right\}$ ) at a sequence of times commencing at the time $T_{u}^{A}$. This explains the additional term " $\log d_{A}(x)$ " in the expression for $S_{0}$; namely, it arises when transforming $V_{T_{u}^{A}}^{A}$ to $V_{T_{u}^{A}}$. 
As a corollary, we specialize to the case where $g=1$ [in (4.2)], and then to the case where we also have $d_{A}=1$ [in (4.3)]. We use the shorthand notation $C(v)=$ $r_{\alpha}(\widetilde{v}) \mathbb{E}_{\delta_{v}}^{\alpha}\left[|Z|^{\alpha} \mathbf{1}_{\{\tau=\infty\}}\right]$ (which is equivalent to the definition given in Section 2) and employ the change of measure in the second identity [namely (4.3)].

COROLLARY 4.2. Under the assumptions of Proposition 4.1, we have that for any $v \in \mathbb{R}_{+}^{d} \backslash\{0\}$,

$$
\lim _{u \rightarrow \infty} u^{\alpha} \mathbb{P}\left(T_{u}^{A}<\tau \mid V_{0}=v\right)=C(v) \int \frac{e^{-\alpha s}}{r_{\alpha}^{A}(x)} \varrho^{A}(d x, d s)
$$

and

$$
\begin{aligned}
\lim _{u \rightarrow \infty} u^{\alpha} \mathbb{E}\left[g\left(\frac{V_{T_{u}}}{u}, \ldots, \frac{V_{T_{u}+m}}{u}\right) \mathbf{1}_{\left\{T_{u}<\tau\right\}} \mid V_{0}=v\right] \\
=C(v) \int \mathbb{E}_{x}^{\alpha}\left[\frac{e^{-\alpha\left(S_{m}+s\right)}}{r_{\alpha}\left(X_{m}\right)} g\left(e^{s} X_{0}, \ldots, e^{S_{m}+s} X_{m}\right)\right] \varrho(d x, d s) .
\end{aligned}
$$

4.2. Proof of Proposition 4.1. We will rely on the following.

Lemma 4.3. Assume the conditions of Proposition 4.1. Then:

(i) For all $v \in \mathbb{R}_{+}^{d} \backslash\{0\}$, we have the $L^{1}$-convergence

$$
\lim _{n \rightarrow \infty} \lim _{u \rightarrow \infty} \mathbb{E}_{\boldsymbol{\delta}_{v}}^{\alpha}\left[\left.|| Z_{T_{u}^{A}}\right|^{\alpha} \mathbf{1}_{\left\{T_{u}^{A}<\tau\right\}}-\left|Z_{n}\right|^{\alpha} \mathbf{1}_{\left\{n \leq T_{u}^{A}\right\}} \mathbf{1}_{\{n \leq \tau\}} \mid\right]=0 .
$$

(ii) For $u>0$, define

$$
\mathfrak{G}_{u}=\frac{1}{r_{\alpha}\left(X_{T_{u}^{A}}\right)}\left(\frac{\left|V_{T_{u}^{A}}\right|}{u}\right)^{-\alpha} \mathbb{E}\left[g\left(\frac{V_{T_{u}^{A}}}{u}, \ldots, \frac{V_{T_{u}^{A}+m}}{u}\right) \mid \mathscr{F}_{T_{u}^{A}}\right] .
$$

Then, independent of $n$, we have $\mathbb{P}^{\alpha}$-a.s. that

$$
\begin{aligned}
\lim _{u \rightarrow \infty} & \mathbb{E}^{\alpha}\left[\mathfrak{G}_{u} \mid \mathscr{F}_{n}\right] \mathbf{1}_{\left\{n \leq T_{u}^{A}\right\}} \\
& =\int \frac{e^{-\alpha s}}{r_{\alpha}^{A}(x)} \mathbb{E}\left[g\left(\left(e^{S_{n}} X_{n}\right)_{n=0}^{m}\right) \mid X_{0}=x, S_{0}=s+\log d_{A}(x)\right] \varrho^{A}(d x, d s) .
\end{aligned}
$$

ProOF. (i) By Lemma 3.2, $\tau$ satisfies the assumptions in Lemma 3.6(iii). Thus, this result is a direct consequence of Lemma 3.6(iv), where the $L^{1}$ convergence $\left|Z_{n}\right|^{\alpha} \mathbf{1}_{\{n \leq \tau\}} \rightarrow|Z|^{\alpha} \mathbf{1}_{\{\tau=\infty\}}$ is proved. It follows that $\left|Z_{n}\right|^{\alpha} \mathbf{1}_{\{n \leq \tau\}}$ constitutes a Cauchy sequence in $L^{1}$, yielding the assertion.

(ii) Let $n \in \mathbb{N}_{+}$. Then by the Markov property,

$$
\mathbb{E}^{\alpha}\left[\mathfrak{G}_{u} \mid \mathscr{F}_{n}\right] \mathbf{1}_{\left\{n \leq T_{u}^{A}\right\}}=\mathbb{E}_{X_{n}, V_{n}}^{\alpha}\left[\mathfrak{G}_{u}\right] \mathbf{1}_{\left\{n \leq T_{u}^{A}\right\}} \quad \mathbb{P}^{\alpha} \text {-a.s. }
$$


As $\lim _{u \rightarrow \infty} \mathbf{1}_{\left\{n \leq T_{u}^{A}\right\}}=1 \mathbb{P}^{\alpha}$-a.s., it suffices to determine $\lim _{u \rightarrow \infty} \mathbb{E}_{x, v}^{\alpha}\left[\mathfrak{G}_{u}\right]$ and show that this quantity is independent of $x$ and $v$.

For all $v \in \mathbb{R}_{+}^{d}$ and $u>0$, set

$$
G_{u}(v)=\mathbb{E}\left[g\left(v, \ldots, \Pi_{m} v+\frac{V_{m}^{(0)}}{u}\right)\right], \quad G(v)=\mathbb{E}\left[g\left(v, \Pi_{1} v, \ldots, \Pi_{m} v\right)\right],
$$

where $V_{m}^{(0)}:=\sum_{i=1}^{m} \Pi_{i+1}^{m} Q_{i}$ for $m \geq 2$ and $V_{1}^{(0)}:=Q_{1}$. Now consider the decomposition:

$$
\begin{aligned}
\mathbb{E}_{x, v}^{\alpha}\left[\mathfrak{G}_{u}\right]= & \mathbb{E}_{x, v}^{\alpha}\left[\frac{1}{r_{\alpha}\left(X_{T_{u}^{A}}\right)}\left(\frac{\left|V_{T_{u}^{A}}\right|}{u}\right)^{-\alpha}\left(G_{u}\left(\frac{V_{T_{u}^{A}}}{u}\right)-G\left(\frac{V_{T_{u}^{A}}}{u}\right)\right)\right] \\
& +\mathbb{E}_{x, v}^{\alpha}\left[\frac{r_{\alpha}\left(\widetilde{V}_{T_{u}^{A}}\right)}{r_{\alpha}\left(X_{T_{u}^{A}}\right)} \frac{1}{r_{\alpha}\left(\widetilde{V}_{\left.T_{u}^{A}\right)}\right.}\left(\frac{\left|V_{T_{u}^{A}}\right|}{u}\right)^{-\alpha} G\left(\frac{V_{T_{u}^{A}}}{u}\right)\right]:=\mathbb{I}_{1}(u)+\mathbb{I}_{2}(u) .
\end{aligned}
$$

STEP 1 . We begin by showing that $\mathbb{I}_{1}(u) \rightarrow 0$ as $u \rightarrow \infty$. Let $\hat{g}$ be a $\theta$-Hölder continuous function with $g\left(v_{0}, \ldots, v_{m}\right)=\hat{g}\left(v_{0}, \ldots, v_{m}\right) \mathbf{1}_{\left\{\left|v_{m}\right| \geq \delta\right\}}$. Then

$$
\begin{aligned}
\left|g\left(\left(\Pi_{n} v+\left(V_{n}^{(0)} / u\right)\right)_{n=0}^{m}\right)-g\left(\left(\Pi_{n} v\right)_{n=0}^{m}\right)\right| \\
\leq\left|\hat{g}\left(\left(\Pi_{n} v+\left(V_{n}^{(0)} / u\right)\right)_{n=0}^{m}\right)-\hat{g}\left(\left(\Pi_{n} v\right)_{n=0}^{m}\right)\right| \mathbf{1}_{\left\{\left|\Pi_{m} v\right| \geq \delta\right\}} \\
\quad+|g|_{\infty}\left(\mathbf{1}_{[\delta, \infty)}\left(\left|\Pi_{m} v+\left(V_{m}^{(0)} / u\right)\right|\right)-\mathbf{1}_{[\delta, \infty)}\left(\left|\Pi_{m} v\right|\right)\right) \\
\leq \frac{1}{u^{\theta}} B_{1} \sum_{n=1}^{m}\left|V_{n}^{(0)}\right|^{\theta}+|g|_{\infty}\left(\mathbf{1}_{[\delta, \infty)}\left(\left|\Pi_{m} v+\left(V_{m}^{(0)} / u\right)\right|\right)-\mathbf{1}_{[\delta, \infty)}\left(\left|\Pi_{m} v\right|\right)\right)
\end{aligned}
$$

for some constant $B_{1}$ arising from the $\theta$-Hölder continuity of $\hat{g}$. Let $\left(M^{*}, Q^{*}\right)$ be a pair of random variables that is independent of the sequence $\left\{\left(M_{n}, Q_{n}\right)\right\}$, where the $\mathbb{P}^{\alpha}$-law of $\left(M^{*}, Q^{*}\right)$ is given by $\mathbb{P}\left(\left(\Pi_{m}, V_{m}^{(0)}\right) \in \cdot\right)$. Upon setting $B_{2}=$ $\max _{y \in \mathbb{S}_{+}^{d-1}}\left(r_{\alpha}(y)\right)^{-1}$ and using that $\left(\left|V_{T_{u}^{A}}\right| / u\right)^{-\alpha}<1$, we obtain that

$$
\begin{aligned}
\mathbb{I}_{1}(u) \leq & \frac{1}{u^{\theta}} B_{1} B_{2} \mathbb{E}\left[\sum_{n=1}^{m}\left|V_{n}^{(0)}\right|^{\theta}\right] \\
& +B_{2}|g|_{\infty}\left(\mathbb{P}_{x, v}^{\alpha}\left(\left|M^{*} \frac{V_{T_{u}^{A}}}{u}+\frac{Q^{*}}{u}\right| \geq \delta\right)\right. \\
& \left.-\mathbb{P}_{x, v}^{\alpha}\left(\left|M^{*} \frac{V_{T_{u}^{A}}}{u}\right| \geq \delta\right)\right) .
\end{aligned}
$$

Since the $\theta$-moment of $V_{n}^{(0)}$ is finite, the first term tends to zero as $u \rightarrow$ $\infty$. For the second term, use the $\mathbb{P}^{\alpha}$-convergence $\left(M^{*}, Q^{*} / u\right) \Rightarrow\left(M^{*}, 0\right)$ and $\left(\widetilde{V}_{T_{u}^{A}}^{A}, \log \left|V_{T_{u}^{A}}^{A}\right|-\log u\right) \Rightarrow \varrho^{A}$ (by Theorem 3.8). Let $(X, S) \sim \varrho^{A}$ be a random vector independent of $\left(M^{*}, Q^{*}\right)$ under $\mathbb{P}^{\alpha}$. Solving (3.32) for $V_{n}$, we have that 
$V_{T_{u}^{A}} / u \Rightarrow d_{A}(X) e^{S} X$. (Here, $X$ describes the limiting direction of $V_{T_{u}^{A}}^{A} / u$ and $S$ the limiting logarithmic overjump, as $\log \left|V_{T_{u}^{A}}^{A}\right|-\log u \Rightarrow S$.) Since the sequences $\left\{\left(M^{*}, Q^{*} / u\right)\right\}$ and $\left\{V_{T_{u}^{A}} / u\right\}$ are independent, they converge jointly in distribution. Hence, under $\mathbb{P}^{\alpha}$,

$$
M^{*} \frac{V_{T_{u}^{A}}}{u}+\frac{Q^{*}}{u} \Rightarrow d_{A}(X) e^{S} M^{*} X \quad \text { and } \quad M^{*} \frac{V_{T_{u}^{A}}}{u} \Rightarrow d_{A}(X) e^{S} M^{*} X .
$$

Thus, the second term in (4.6) vanishes if $[\delta, \infty)$ is a continuity set for $d_{A}(X) \times$ $e^{S}\left|M^{*} X\right|$.

We now show that $[\delta, \infty)$ is a continuity set. Since $M^{*}$ is independent of $(X, S)$, it suffices to show that for any allowable matrix $\mathfrak{m}$, the event $\left\{d_{A}(X) e^{S}|\mathfrak{m} X|=\delta\right\}$ has probability 0 . Now for each fixed $y \in \mathbb{S}_{+}^{d-1}$, the equation $\mathfrak{h}(s):=d_{A}(y) e^{s}|\mathfrak{m} y|=\delta$ has a unique solution $s_{y} \in \mathbb{R}$. Hence

$$
\mathbb{P}^{\alpha}\left(d_{A}(X) e^{S}|\mathfrak{m} X|=\delta\right)=\int_{\mathbb{S}_{+}^{d-1} \times \mathbb{R}} \mathbf{1}_{\left\{s=s_{y}\right\}} \varrho^{A}(d y, d s)=0,
$$

since the radial component of the overjump distribution is absolutely continuous with respect to Lebesgue measure (as can be seen from the representation of $\varrho^{A}$ in equation (1.16) of [29]).

Thus, having shown that $[\delta, \infty)$ is a continuity set, we conclude by the Portmanteau theorem that for all $x \in \mathbb{S}_{+}^{d-1}$ and $v \in \mathbb{R}_{+}^{d} \backslash\{0\}$,

$$
\begin{aligned}
& \mathbb{P}_{x, v}^{\alpha}\left(\left|M^{*} \frac{V_{T_{u}^{A}}}{u}+\frac{Q^{*}}{u}\right| \geq \delta\right)-\mathbb{P}_{x, v}^{\alpha}\left(\left|M^{*} \frac{V_{T_{u}^{A}}}{u}\right| \geq \delta\right) \\
& \quad \rightarrow \mathbb{P}^{\alpha}\left(d_{A}(X) e^{S}\left|M^{*} X\right| \geq \delta\right)-\mathbb{P}^{\alpha}\left(d_{A}(X) e^{S}\left|M^{*} X\right| \geq \delta\right) .
\end{aligned}
$$

Hence the second member of (4.6) also vanishes as $u \rightarrow \infty$. Thus $\mathbb{I}_{1}(u) \rightarrow 0$ as $u \rightarrow \infty$.

STEP 2. Now turn to $\mathbb{I}_{2}(u)$. Using Theorem 3.8, again invoke the convergence $\left(\left(V_{T_{u}^{A}}^{A}\right)^{\sim}, \log \left|V_{T_{u}^{A}}^{A}\right|-\log u\right) \Rightarrow \varrho^{A}$ under $\mathbb{P}^{\alpha}$. Moreover, by Lemma 3.10, using the continuity and boundedness of $r_{\alpha}$, we have that $r_{\alpha}\left(\widetilde{V}_{T_{u}}\right) / r_{\alpha}\left(X_{T_{u}}\right)$ tends to one in $\mathbb{P}^{\alpha}$-probability. Hence by Slutsky's theorem, the quantity inside $\mathbb{I}_{2}(u)$ converges in law, and identifying this limit distribution, we deduce that

$$
\begin{aligned}
\lim _{u \rightarrow \infty} \mathbb{I}_{2}(u) & =\lim _{u \rightarrow \infty} \mathbb{E}_{x, v}^{\alpha}\left[\frac{r_{\alpha}\left(\widetilde{V}_{T_{u}^{A}}\right)}{r_{\alpha}\left(X_{T_{u}^{A}}\right)} \frac{1}{r_{\alpha}^{A}\left(\widetilde{V}_{T_{u}^{A}}^{A}\right)}\left(\frac{\left|V_{T_{u}^{A}}^{A}\right|}{u}\right)^{-\alpha} G\left(\frac{V_{T_{u}^{A}}}{u}\right)\right] \\
& =\int_{\mathbb{S}_{+}^{d-1} \times \mathbb{R}_{+}} \frac{e^{-\alpha s}}{r_{\alpha}^{A}(y)} G\left(d_{A}(y) e^{s} y\right) \varrho^{A}(d y, d s) .
\end{aligned}
$$

Recalling that $G(v)=\mathbb{E}\left[g\left(v, \Pi_{1} v, \ldots, \Pi_{m} v\right)\right]$, the assertion follows.

Proof OF Proposition 4.1. Note that $\left\{V_{n}\right\}$ is transient in the $\alpha$-shifted measure, and thus $T_{u}^{A}<\infty$ a.s.; cf. Lemma 3.12. Hence, employing the change of 
measure only over the random time interval $\left[0, T_{u}^{A}\right]$ (namely, the "dual" change of measure of [18], Section 4, which we denote by the superscript $\mathscr{D}$ ), we obtain that

$$
\begin{aligned}
u^{\alpha} \mathbb{E} & {\left[g\left(\frac{V_{T_{u}^{A}}}{u}, \ldots, \frac{V_{T_{u}^{A}+m}}{u}\right) \mathbf{1}_{\left\{T_{u}^{A}<\tau\right\}} \mid V_{0}=v\right] } \\
& =u^{\alpha} r_{\alpha}(\widetilde{v}) \mathbb{E}_{\boldsymbol{\delta}_{v}}^{\mathscr{D}}\left[\frac{e^{-\alpha S_{T_{u}^{A}}}}{r_{\alpha}\left(X_{T_{u}^{A}}\right)} \mathbf{1}_{\left\{T_{u}^{A}<\tau\right\}} \mathbb{E}\left[g\left(\frac{V_{T_{u}^{A}}}{u}, \ldots, \frac{V_{T_{u}^{A}+m}}{u}\right) \mid \mathscr{F}_{T_{u}^{A}}\right]\right] .
\end{aligned}
$$

Now substitute the quantity $\mathfrak{G}_{u}$ of Lemma 4.3(ii) into the previous equation. Noting that $Z_{n}=V_{n} /\left|\Pi_{n} X_{0}\right|=\left(V_{n} / e^{S_{n}}\right), n \in \mathbb{N}_{+}$, we obtain after a little algebra that

$$
\begin{aligned}
u^{\alpha} \mathbb{E}\left[g\left(\frac{V_{T_{u}^{A}}}{u}, \ldots, \frac{V_{T_{u}^{A}+m}}{u}\right) \mathbf{1}_{\left\{T_{u}^{A}<\tau\right\}} \mid X_{0}=\widetilde{v}, V_{0}=v\right] \\
=r_{\alpha}(\widetilde{v}) \mathbb{E}_{\boldsymbol{\delta}_{v}}^{\mathscr{D}}\left[\left|Z_{T_{u}^{A}}\right|^{\alpha} \mathfrak{G}_{u} \mathbf{1}_{\left\{T_{u}^{A}<\tau\right\}}\right] .
\end{aligned}
$$

For $n \in \mathbb{N}_{+}$, the right-hand side can be further equated to

$$
\begin{gathered}
r_{\alpha}(\widetilde{v}) \mathbb{E}_{\boldsymbol{\delta}_{v}}^{\alpha}\left[\left(\left|Z_{T_{u}^{A}}\right|^{\alpha} \mathbf{1}_{\left\{T_{u}^{A}<\tau\right\}}-\left|Z_{n}\right|^{\alpha} \mathbf{1}_{\left\{n \leq T_{u}^{A}\right\}} \mathbf{1}_{\{n \leq \tau\}}\right) \mathfrak{G}_{u}\right] \\
+r_{\alpha}(\widetilde{v}) \mathbb{E}_{\delta_{v}}^{\alpha}\left[\left|Z_{n}\right|^{\alpha} \mathbf{1}_{\left\{n \leq T_{u}^{A}\right\}} \mathbf{1}_{\{n \leq \tau\}} \mathbb{E}^{\alpha}\left[\mathfrak{G}_{u} \mid \mathscr{F}_{n}\right]\right],
\end{gathered}
$$

where we have replaced $\mathbb{E}_{\boldsymbol{\delta}_{v}}^{\alpha}\left[\cdot \mid \mathscr{F}_{n}\right]$ with $\mathbb{E}^{\alpha}\left[\cdot \mid \mathscr{F}_{n}\right]$ in the last expectation, since this conditional expectation depends only on $\left(X_{n}, V_{n}\right)$, and not on the initial values $\left(X_{0}, V_{0}\right)$ once $\left(X_{n}, V_{n}\right)$ has been specified. Moreover, the superscript $\mathscr{D}$ can now be dropped, since the change of measure over the random time interval $\left[0, T_{u}^{A}\right]$ coincides with the usual $\alpha$-shifted measure for $\mathscr{F}_{T_{u}^{A}}$-measurable random variables.

To analyze the quantity in (4.8), we first take the limit as $u \rightarrow \infty$ and then as $n \rightarrow \infty$. By part (i) of Lemma 4.3 and the boundedness of $\mathfrak{G}_{u}$, we deduce from (4.7) and (4.8) that

$$
\begin{aligned}
\lim _{u \rightarrow \infty} u^{\alpha} \mathbb{E}\left[g\left(\frac{V_{T_{u}^{A}}}{u}, \ldots, \frac{V_{T_{u}^{A}+m}}{u}\right) \mathbf{1}_{\left\{T_{u}^{A}<\tau\right\}} \mid V_{0}=v\right] \\
=\lim _{n \rightarrow \infty} \lim _{u \rightarrow \infty} r_{\alpha}(\widetilde{v}) \mathbb{E}_{\boldsymbol{\delta}_{v}}^{\alpha}\left[\left|Z_{n}\right|^{\alpha} \mathbf{1}_{\left\{n \leq T_{u}^{A}\right\}} \mathbf{1}_{\{n \leq \tau\}} \mathbb{E}^{\alpha}\left[\mathfrak{G}_{u} \mid \mathscr{F}_{n}\right]\right] .
\end{aligned}
$$

Now by Lemma 3.6(iii), $\left\{\left|Z_{n}\right|^{\alpha} \mathbf{1}_{\{n \leq \tau\}}\right\}$ is uniformly integrable. Denote by $\mathfrak{G}$ the right-hand side of (4.5). Since $\mathfrak{G}_{u}$ is bounded by $b^{-1}|g|_{\infty}$ and $T_{u}^{A} \uparrow \infty \mathbb{P}^{\alpha}$-a.s., it follows by Lemma 4.3(ii) that

$$
\begin{aligned}
\lim _{u \rightarrow \infty} & u^{\alpha} \mathbb{E}\left[g\left(\frac{V_{T_{u}^{A}}}{u}, \ldots, \frac{V_{T_{u}^{A}+m}}{u}\right) \mathbf{1}_{\left\{T_{u}^{A}<\tau\right\}} \mid V_{0}=v\right] \\
= & \lim _{n \rightarrow \infty} r_{\alpha}(\widetilde{v}) \mathbb{E}_{\boldsymbol{\delta}_{v}}^{\alpha}\left[\left|Z_{n}\right|^{\alpha} \mathbf{1}_{\{n \leq \tau\}} \lim _{u \rightarrow \infty} \mathbf{1}_{\left\{n \leq T_{u}^{A}\right\}} \mathbb{E}^{\alpha}\left[\mathfrak{G}_{u} \mid \mathscr{F}_{n}\right]\right] \\
= & r_{\alpha}(\widetilde{v}) \mathbb{E}_{\boldsymbol{\delta}_{v}}^{\alpha}\left[\lim _{n \rightarrow \infty}\left|Z_{n}\right|^{\alpha} \mathbf{1}_{\{n \leq \tau\}} \mathfrak{G}\right]=r_{\alpha}(\widetilde{v}) \mathbb{E}_{\boldsymbol{\delta}_{v}}^{\alpha}\left[|Z|^{\alpha} \mathbf{1}_{\{\tau=\infty\}}\right] \mathfrak{G} .
\end{aligned}
$$


In some cases, it is useful to consider functions $g$ which depend on the infinite path $\left(V_{T_{u}^{A}}, V_{T_{u}^{A}+1}, \ldots\right)$, or to consider functions $g$ which need not be bounded. Moreover, it is also useful to have uniform upper bounds. In these situations, a variant of the above proposition is useful.

Proposition 4.4. Suppose that $g:\left(\mathbb{R}_{+}^{d}\right)^{\mathbb{N}} \rightarrow[0, \infty)$ is a nonnegative measurable function, and set

$$
\overline{\mathfrak{G}}_{u}=\frac{1}{r_{\alpha}\left(X_{T_{u}^{A}}\right)}\left(\frac{\left|V_{T_{u}^{A}}\right|}{u}\right)^{-\alpha} \mathbb{E}\left[g\left(\left(\frac{V_{T_{u}^{A}+k}}{u}\right)_{k \geq 0}\right) \mid \mathscr{F}_{T_{u}^{A}}\right] .
$$

Further, assume that for some finite constant $B$ and some $\mathscr{U} \geq 0$,

$$
\sup _{u \geq \mathscr{U}} \overline{\mathfrak{G}}_{u} \leq B \quad \mathbb{P}^{\alpha} \text {-a.s. }
$$

Then for any bounded set $F \subset \mathbb{R}_{+}^{d} \backslash\{0\}$, there exists a finite constant $L$, not depending on $B$, such that

$$
0 \leq \sup _{u \geq \mathscr{U}} \sup _{v \in F} u^{\alpha} \mathbb{E}\left[g\left(\left(\frac{V_{T_{u}^{A}+k}}{u}\right)_{k \geq 0}\right) \mathbf{1}_{\left\{T_{u}^{A}<\tau\right\}} \mid V_{0}=v\right] \leq B L .
$$

Moreover, if (4.11) holds and $\lim \sup _{u \rightarrow \infty} \overline{\mathfrak{G}}_{u}=0 \mathbb{P}^{\alpha}$-a.s., then

$$
\lim _{u \rightarrow \infty} u^{\alpha} \mathbb{E}\left[g\left(\left(\frac{V_{T_{u}^{A}+k}}{u}\right)_{k \geq 0}\right) \mathbf{1}_{\left\{T_{u}^{A}<\tau\right\}} \mid V_{0}=v\right]=0 .
$$

PROOF. Repeating the argument in the proof of Proposition 4.1 leading to (4.9), we obtain that

$$
\begin{aligned}
0 & \leq \sup _{u \geq \mathscr{U}} \sup _{v \in F} u^{\alpha} \mathbb{E}\left[g\left(\left(\frac{V_{T_{u}^{A}+k}}{u}\right)_{k \geq 0}\right) \mathbf{1}_{\left\{T_{u}^{A}<\tau\right\}} \mid V_{0}=v\right] \\
& \leq B r_{\alpha}(\widetilde{v}) \sup _{v \in F} \mathbb{E}_{\delta_{v}}^{\alpha}\left[\sup _{n \in \mathbb{N}}\left|Z_{n}\right|^{\alpha} \mathbf{1}_{\{n \leq \tau\}}\right],
\end{aligned}
$$

which is finite by Lemma 3.6(iii) and the boundedness of $r_{\alpha}$. The boundedness of $\overline{\mathfrak{G}}_{u}$ then allows us to use the dominated convergence theorem in order to deduce (4.13) from (4.9).

4.3. Toward the proof of Theorem 2.4. We now restrict our attention to the case where $d_{A}=1$; thus $T_{u}^{A}=T_{u}, r_{\alpha}^{A}=r_{\alpha}$, and $\varrho^{A}=\varrho$.

In order to establish Theorem 2.4 in the subsequent section, we first prove a proposition which, together with Lemma 3.5, will link the tail properties of $V$ to the renewal measure associated with $\left\{\left(X_{n}, S_{n}\right)\right\}$. As before, we use the shorthand notation $C(v)=r_{\alpha}(\widetilde{v}) \mathbb{E}_{\delta_{v}}^{\alpha}\left[|Z|^{\alpha} \mathbf{1}_{\{\tau=\infty\}}\right]$. 
Proposition 4.5. Assume $\left(\mathrm{H}_{1}\right)$ and $\left(\mathrm{H}_{2}\right)$ are satisfied. Let $\theta \leq \min \{1, \alpha\}$ and let $f$ be a nonnegative bounded $\theta$-Hölder continuous function. Then for all $v \in \mathbb{R}_{+}^{d} \backslash\{0\}$,

$$
\begin{aligned}
\lim _{u \rightarrow \infty} u^{\alpha} \mathbb{E}\left[\sum_{i=0}^{\tau-1} f\left(\frac{V_{i}}{u}\right) \mathbf{1}_{\left\{\left|V_{i}\right| \geq u\right\}} \mid V_{0}=v\right] \\
=C(v) \int_{\mathbb{S}_{+}^{d-1} \times \mathbb{R}_{+}} \mathbb{E}_{x}^{\alpha}\left[\sum_{i=0}^{\infty} F\left(X_{i}, S_{i}+s\right)\right] \varrho(d x, d s),
\end{aligned}
$$

where $F(x, s):=\left(e^{-\alpha s} f\left(e^{s} x\right) / r_{\alpha}(x)\right) \mathbf{1}_{[0, \infty)}(s)$ is directly Riemann integrable.

We note by [36], Section 6.1, that if $F$ is directly Riemann integrable [as defined in [10], equation (7.1)], it follows that on the right-hand side of (4.14)

$$
\sup _{x \in \mathbb{S}_{+}^{d-1}} \sup _{s \in \mathbb{R}} \mathbb{E}_{x}^{\alpha}\left[\sum_{i=0}^{\infty}\left|F\left(X_{i}, S_{i}+s\right)\right|\right]<\infty .
$$

We shall deduce Proposition 4.5 from Corollary 4.2. However, to do so, we need to handle the remainder terms, which we study in the following.

LEMMA 4.6. Let $h$ be a bounded measurable function such that $h(x)=0$ for all $x \in B_{\delta}^{+}(0)$, for some $\delta>0$. Then for all $v \in \mathbb{R}_{+}^{d} \backslash\{0\}$ and all $m \in \mathbb{N}$,

$$
\lim _{m \rightarrow \infty} \lim _{u \rightarrow \infty} u^{\alpha} \mathbb{E}\left[\sum_{k=m}^{\tau-1-T_{u}} h\left(\frac{V_{T_{u}+k}}{u}\right) \mathbf{1}_{\left\{T_{u}+m<\tau\right\}} \mid V_{0}=v\right]=0 .
$$

Moreover, if $F \subset \mathbb{R}_{+}^{d} \backslash\{0\}$ is bounded, then

$$
\limsup _{u \rightarrow \infty} \sup _{v \in F} u^{\alpha} \mathbb{E}\left[\sum_{i=0}^{\tau-1} h\left(\frac{V_{i}}{u}\right) \mathbf{1}_{\left\{\left|V_{i}\right|>u\right\}} \mid V_{0}=v\right]<\infty .
$$

Furthermore, for all $v \in \mathbb{R}_{+}^{d} \backslash\{0\}$,

$$
\lim _{m \rightarrow \infty} \lim _{u \rightarrow \infty} u^{\alpha} \mathbb{E}\left[\sum_{i=\tau}^{T_{u}+m} h\left(\frac{V_{i}}{u}\right) \mathbf{1}_{\left\{T_{u}<\tau \leq T_{u}+m\right\}} \mid V_{0}=v\right]=0 .
$$

PRoOF. STEP 1. First, we establish (4.16).

By equation (4.12) in Proposition 4.4, it suffices to prove that

$$
\sup _{u \geq \mathscr{U}} \overline{\mathfrak{G}}_{u}:=\sup _{u \geq \mathscr{U}} \frac{1}{r_{\alpha}\left(X_{T_{u}}\right)}\left(\frac{\left|V_{T_{u}}\right|}{u}\right)^{-\alpha} \mathbb{E}\left[\sum_{k=m}^{\tau-1-T_{u}}\left|h\left(\frac{V_{T_{u}+k}}{u}\right)\right| \mathbf{1}_{\left\{T_{u}+m<\tau\right\}} \mid \mathscr{F}_{T_{u}}\right]
$$

is bounded above by $B(m, \mathscr{U})$, where the sequence $\{B(m, \mathscr{U})\}$ tends to zero as we first let $\mathscr{U} \rightarrow \infty$ and then let $m \rightarrow \infty$. By employing the Markov property and the 
boundedness of $r_{\alpha}$, we see that it is enough to show that, for a suitable sequence $B(m, \mathscr{U})$,

$$
\sup _{u \geq \mathscr{U}} \sup _{v:|v| \geq u} H_{u}(v)
$$

$$
\begin{aligned}
& :=\sup _{u \geq \mathscr{U} v:|v| \geq u} \sup _{v} \mathbb{E}\left[\left(\frac{\left|V_{0}\right|}{u}\right)^{-\alpha} \sum_{k=m}^{\tau-1} h\left(\frac{V_{k}}{u}\right) \mathbf{1}_{\{m<\tau\}} \mid V_{0}=v\right] \\
& \leq B(m, \mathscr{U}) .
\end{aligned}
$$

Now let $\mathbb{D}^{\dagger}=\left\{v \in \mathbb{R}_{+}^{d}:|v| \leq L\right\}$ be defined as in Lemma 3.1, and set $\tau^{\dagger}:=$ $\inf \left\{n \in \mathbb{N}_{+}: V_{n} \in \mathbb{D}^{\dagger}\right\}$. Recall that $h(x)=0$ for all $x \in B_{\delta}^{+}(0)$. Hence, for $0<\theta<\min \{1, \alpha\}$ and $|v|>u$,

$$
\begin{aligned}
H_{u}(v) \leq & |h|_{\infty} \mathbb{E}\left[\left(\frac{\left|V_{0}\right|}{u}\right)^{-\alpha}\left(\sum_{k=m}^{\tau^{\dagger}-1} \mathbf{1}_{\left\{\left|V_{k}\right|>\delta u\right\}}+\sum_{k=\tau^{\dagger}}^{\tau-1} \mathbf{1}_{\left\{\left|V_{k}\right|>\delta u\right\}}\right) \mid V_{0}=v\right] \\
\leq & |h|_{\infty}\left(\frac{|v|}{u}\right)^{-\alpha} \sum_{k=m}^{\infty}(\delta u)^{-\theta} \mathbb{E}_{v}\left[\left|V_{k}\right|^{\theta} \mathbf{1}_{\left\{\tau^{\dagger}>k\right\}}\right] \\
& +|h|_{\infty} \sup _{w \in \mathbb{D}^{\dagger}} \mathbb{E}_{w}\left[\sum_{k=0}^{\tau} \mathbf{1}_{\left\{\left|V_{k}\right|>\delta u\right\}}\right] .
\end{aligned}
$$

The first sum can be estimated further by employing Lemma 3.1, namely,

$$
\begin{aligned}
\sup _{v:|v| \geq u} & \left(\frac{|v|}{u}\right)^{-\alpha} \sum_{k=m}^{\infty}(\delta u)^{-\theta} \mathbb{E}_{v}\left[\left|V_{k}\right|^{\theta} \mathbf{1}_{\left\{\tau^{\dagger}>k\right\}}\right] \\
\leq & \frac{B}{\delta^{\theta}}\left(\sup _{v:|v| \geq u}\left(\frac{|v|}{u}\right)^{\theta-\alpha}\right) \frac{t^{m}}{1-t}=\frac{B}{\delta^{\theta}} \frac{t^{m}}{1-t},
\end{aligned}
$$

and this last term tends to zero as $m \rightarrow \infty$. For the second term, note that equation (3.4) of Lemma 3.2 implies that $\sup _{w \in \mathbb{D}^{\dagger}} \mathbb{E}\left[\tau \mid V_{0}=w\right]<\infty$. Hence, we can apply a dominated convergence argument to infer that

$$
\sup _{u \geq \mathscr{U}} \sup _{w \in \mathbb{D}^{\dagger}} \mathbb{E}_{w}\left[\sum_{k=0}^{\tau} \mathbf{1}_{\left\{\left|V_{k}\right|>\delta u\right\}}\right] \leq \sup _{w \in \mathbb{D}^{\dagger}} \mathbb{E}_{w}\left[\sum_{k=0}^{\tau} \mathbf{1}_{\left\{\left|V_{k}\right|>\delta \mathscr{U}\right\}}\right] \rightarrow 0 \quad \text { as } \mathscr{U} \rightarrow \infty .
$$

Combining these estimates establishes (4.19), and (4.16) follows.

Finally, (4.17) is a direct consequence of (4.19) (with $m=0$ and $\delta=1$ ) combined with (4.12).

STEP 2. Turning to (4.18), we apply the second part of Proposition 4.4. Using that $h=0$ on $B_{\delta}(0)$, it is now sufficient to show that for any $m \in \mathbb{N}$,

$$
\overline{\mathfrak{G}}_{u}:=\frac{1}{r_{\alpha}\left(X_{T_{u}}\right)}\left(\frac{\left|V_{T_{u}}\right|}{u}\right)^{-\alpha} \mathbb{E}\left[\sum_{i=\tau}^{T_{u}+m}\left|h\left(\frac{V_{i}}{u}\right)\right| \mathbf{1}_{\left\{\left|V_{i}\right|>\delta u\right\}} \mathbf{1}_{\left\{T_{u}<\tau \leq T_{u}+m\right\}} \mid \mathscr{F}_{T_{u}}\right]
$$


is bounded uniformly in $u$ and tends to zero $\mathbb{P}^{\alpha}$-a.s. as $u \rightarrow \infty$. As the prefactors are bounded, it suffices to estimate

$$
\begin{aligned}
& \mathbb{E}\left[\sum_{i=\tau}^{T_{u}+m}\left|h\left(\frac{V_{i}}{u}\right)\right| \mathbf{1}_{\left\{\left|V_{i}\right|>\delta u\right\}} \mathbf{1}_{\left\{T_{u}<\tau \leq T_{u}+m\right\}} \mid \mathscr{F}_{T_{u}}\right] \\
& \quad \leq|h|_{\infty} \mathbb{E}\left[\mathbb{E}\left[\sum_{i=\tau}^{\tau+m} \mathbf{1}_{\left\{\left|V_{i}\right|>\delta u\right\}} \mid \mathscr{F}_{\tau}\right] \mathbf{1}_{\left\{T_{u}<\tau\right\}} \mid \mathscr{F}_{T_{u}}\right] .
\end{aligned}
$$

Let $\theta \in(0, \alpha)$. Then for all $k=0, \ldots, m$,

$$
\begin{aligned}
\mathbb{P}\left(\left|V_{\tau+k}\right|>\delta u \mid V_{\tau}=v\right) & \leq \sup _{v \in \mathbb{D}}(\delta u)^{-\theta} \mathbb{E}\left[\left|V_{k}\right|^{\theta} \mid V_{0}=v\right] \\
& \leq \delta^{-\theta} u^{-\theta}\left(\sup _{v \in \mathbb{D}} \mathbb{E}\left[\left\|\Pi_{k}\right\|^{\theta}\right] \cdot|v|^{\theta}+\sum_{j=1}^{k} \mathbb{E}\left[\left|\Pi_{j+1}^{k} Q_{j}\right|^{\theta}\right]\right) \\
& \leq B_{1} u^{-\theta}
\end{aligned}
$$

for some finite constant $B_{1}$ (dependent on $m$ ). Substituting this estimate into (4.20) and then into the definition of $\overline{\mathfrak{G}}_{u}$ above (4.20), we obtain that $\overline{\mathfrak{G}}_{u} \leq B_{2} m u^{-\theta} \downarrow 0$ as $u \rightarrow \infty$, some $B_{2}<\infty$. Thus, by Proposition 4.4,

$$
\lim _{u \rightarrow \infty} u^{\alpha} \mathbb{E}\left[\sum_{i=\tau}^{T_{u}+m} h\left(\frac{V_{i}}{u}\right) \mathbf{1}_{\left\{T_{u}<\tau \leq T_{u}+m\right\}} \mid V_{0}=v\right]=0 \quad \forall m \in \mathbb{N},
$$

and (4.18) follows.

Proof of Proposition 4.5. Let $g(v):=f(v) \mathbf{1}_{\{|v| \geq 1\}}$, and note that $g$ is an almost $\theta$-Hölder-continuous function. Now

$$
u^{\alpha} \mathbb{E}_{v}\left[\sum_{i=0}^{\tau-1} f\left(\frac{V_{i}}{u}\right) \mathbf{1}_{\left\{\left|V_{i}\right| \geq u\right\}}\right]=u^{\alpha} \mathbb{E}_{v}\left[\sum_{i=T_{u}}^{\tau-1} f\left(\frac{V_{i}}{u}\right) \mathbf{1}_{\left\{\left|V_{i}\right| \geq u\right\}} \mathbf{1}_{\left\{T_{u}<\tau\right\}}\right],
$$

and the right-hand side can be decomposed into three terms, namely

$$
\begin{gathered}
u^{\alpha} \sum_{k=0}^{m} \mathbb{E}_{v}\left[g\left(\frac{V_{T_{u}+k}}{u}\right) \mathbf{1}_{\left\{T_{u}<\tau\right\}}\right]+u^{\alpha} \mathbb{E}_{v}\left[\sum_{k=m+1}^{\tau-1-T_{u}} g\left(\frac{V_{T_{u}+k}}{u}\right) \mathbf{1}_{\left\{T_{u}+m<\tau\right\}}\right] \\
-u^{\alpha} \mathbb{E}_{v}\left[\sum_{i=\tau}^{T_{u}+m} g\left(\frac{V_{i}}{u}\right) \mathbf{1}_{\left\{T_{u}<\tau \leq T_{u}+m\right\}}\right] .
\end{gathered}
$$

On the right-hand side of (4.21), the last two terms tend to zero, by Lemma 4.6, when taking first the limit $u \rightarrow \infty$ and then $m \rightarrow \infty$. Next, by Corollary 4.2, 
equation (4.3), we obtain for the remaining term that

$$
\begin{aligned}
\lim _{u \rightarrow \infty} u^{\alpha} \sum_{k=0}^{m} \mathbb{E}\left[g\left(\frac{V_{T_{u}+k}}{u}\right) \mathbf{1}_{\left\{T_{u}<\tau\right\}} \mid V_{0}=v\right] \\
=C(v) \int_{\mathbb{S}_{+}^{d-1} \times \mathbb{R}_{+}} \mathbb{E}_{x}^{\alpha}\left[\sum_{i=0}^{m} \frac{e^{-\alpha\left(S_{i}+s\right)}}{r_{\alpha}\left(X_{i}\right)} f\left(e^{S_{i}+s} X_{i}\right) \mathbf{1}_{\left\{S_{i}+s \geq 0\right\}}\right] \varrho(d x, d s) .
\end{aligned}
$$

It remains to show that $F(x, s):=\left(e^{-\alpha s} f\left(e^{s} x\right) / r_{\alpha}(x)\right) \mathbf{1}_{[0, \infty)}(s)$ is directly Riemann integrable, which, by (4.15), will allows us to take the limit as $m \rightarrow \infty$. Since $r_{\alpha}$ is bounded from below, it follows that for some positive constant $b$,

$$
\bar{F}(s):=\sup _{x \in \mathbb{S}_{+}^{d-1}}|F(x, s)| \leq \frac{1}{b}|f|_{\infty} e^{-\alpha s} \mathbf{1}_{[0, \infty)}(s) .
$$

Since the right-hand side is a decreasing integrable function, we conclude that $\bar{F}$ is (univariate) directly Riemann integrable. But $\bar{F}$ is obtained from $F$ by taking the supremum over all $x \in \mathbb{S}_{+}^{d-1}$, so it follows immediately that $F$ is (multivariate) Riemann integrable.

5. Proof of Theorem 2.4. In this section, we provide the proof of Theorem 2.4, first under the additional hypothesis $\left(\mathrm{H}_{3}\right)$ of Section 3 , which is then removed by approximating $\left\{V_{n}\right\}$ from above and below by smoothed processes for which $\left(\mathrm{H}_{3}\right)$ is satisfied.

To establish Theorem 2.4, we apply Proposition 4.5 directly, except that we must identify the integral in (4.14). This is done in the following lemma.

LEMMA 5.1. Let $g: \mathbb{S}_{+}^{d-1} \times \mathbb{R} \rightarrow \mathbb{R}$ be a directly Riemann integrable function. Then

$$
\int \mathbb{E}_{x}^{\alpha}\left[\sum_{i=0}^{\infty} g\left(X_{i}, S_{i}+s\right) \mathbf{1}_{\left\{S_{i}+s \geq 0\right\}}\right] \varrho(d x, d s)=\frac{1}{\lambda^{\prime}(\alpha)} \int g(x, s) \eta_{\alpha}(d x) d s .
$$

The crucial point is to identify $\varrho$ as the stationary Markov delay distribution, that is, the initial distribution for $\left\{\left(X_{n}, S_{n}\right)\right\}$ under which the renewal measure (restricted to $\mathbb{S}_{+}^{d-1} \times \mathbb{R}_{+}$) equals $\eta_{\alpha} \otimes d s$. This identification can be done along identical lines to the proofs of Theorem 3 and Corollary 3 in [2]. For this reason, we omit the proof and refer the reader to the arXiv version [16] of our article for the details.

We now establish Theorem 2.4 under the additional Hypothesis $\left(\mathrm{H}_{3}\right)$ of Section 3.

Proposition 5.2. Assume that Hypotheses $\left(\mathrm{H}_{1}\right),\left(\mathrm{H}_{2}\right)$ and $\left(\mathrm{H}_{3}\right)$ are satisfied, and suppose that $\mathbb{D} \in \mathscr{B}\left(\mathbb{R}_{+}^{d}\right)$ is bounded and $\pi(\mathbb{D})>0$. Then for any $f \in \mathscr{C}_{0}\left(\mathbb{R}_{+}^{d} \backslash\right.$ 
$\{0\})$,

$$
\lim _{u \rightarrow \infty} u^{\alpha} \mathbb{E}\left[f\left(\frac{V}{u}\right)\right]=\frac{C}{\lambda^{\prime}(\alpha)} \int_{\mathbb{S}_{+}^{d-1} \times \mathbb{R}} e^{-\alpha s} f\left(e^{s} x\right) l_{\alpha}(d x) d s,
$$

where $C$ is given as in (2.10). Equivalently, we have the weak convergence

$$
\lim _{u \rightarrow \infty} u^{\alpha} \mathbb{P}\left(|V|>t u, \frac{V}{|V|} \in \cdot\right) \Rightarrow \frac{C}{\alpha \lambda^{\prime}(\alpha)} t^{-\alpha} l_{\alpha}(\cdot) \quad \text { for all } t>0 .
$$

PROOF. We first prove the result under the additional assumption that $f$ satisfies $f(x)=\hat{f}(x) \mathbf{1}_{\{|x| \geq r\}}$ for some $r>0$, where $\hat{f}$ is a $\theta$-Hölder continuous function with $\theta \leq \min \{1, \alpha\}$, that is, $f$ is almost $\theta$-Hölder continuous.

STEP 1. First, assume $r=1$, that is, $f(x)=\hat{f}(x) \mathbf{1}_{\{|x| \geq 1\}}$. Since $\left(\mathrm{H}_{3}\right)$ is satisfied, it follows from Lemma 3.5 that

$$
\begin{aligned}
\mathbb{E}\left[\hat{f}\left(\frac{V}{u}\right) \mathbf{1}_{\{|V| \geq u\}}\right] & =\frac{1}{\mathbb{E}_{\pi_{\mathbb{D}}}[\tau]} \int_{\mathbb{D}} \mathbb{E}\left[\sum_{i=0}^{\tau-1} \hat{f}\left(\frac{V_{i}}{u}\right) \mathbf{1}_{\left\{\left|V_{i}\right| \geq u\right\}} \mid V_{0}=v\right] \pi_{\mathbb{D}}(d v) \\
& =\int_{\mathbb{D}} \mathbb{E}\left[\sum_{i=0}^{\tau-1} \hat{f}\left(\frac{V_{i}}{u}\right) \mathbf{1}_{\left\{\left|V_{i}\right| \geq u\right\}} \mid V_{0}=v\right] \pi_{\mathbb{D}}(d v),
\end{aligned}
$$

where $\tau$ denotes the first return time of $\left\{V_{n}\right\}$ to $\mathbb{D}$. Moreover, from Lemma 3.3 we have that $\pi(\mathbb{D})=\left(\mathbb{E}_{\pi_{\mathbb{D}}}[\tau]\right)^{-1}$, where $\pi_{\mathbb{D}}(\cdot)=\pi(\cdot \cap \mathbb{D}) / \pi(\mathbb{D})$.

Now apply Proposition 4.5 and the identity (5.3) separately to the positive and negative parts of $\hat{f}$ to obtain that

$$
\begin{aligned}
\lim _{u \rightarrow \infty} & u^{\alpha} \mathbb{E}\left[\hat{f}\left(\frac{V}{u}\right) \mathbf{1}_{\{|V| \geq u\}}\right] \\
= & \int_{\mathbb{D}} C(v)\left(\int_{\mathbb{S}_{+}^{d-1} \times \mathbb{R}_{+}} \mathbb{E}_{x}^{\alpha}\left[\sum_{i=0}^{\infty} F\left(X_{i}, S_{i}+s\right)\right] \varrho(d x, d s)\right) \pi(d v),
\end{aligned}
$$

where $F(x, s)=\left(e^{-\alpha s} f\left(e^{s} x\right) / r_{\alpha}(x)\right) \mathbf{1}_{[0, \infty)}$. Note that Proposition 4.5 actually holds conditional on $\left\{V_{0}=v\right\}$, where $v \in \mathbb{R}_{+}^{d} \backslash\{0\}$; and to extend this result so that it holds conditional on $\left\{V_{0} \sim \pi_{\mathbb{D}}\right\}$, we have applied a dominated convergence argument together with the bound provided by (4.17) of Lemma 4.6. Moreover, we have used that $\pi(\{0\})=0$, which follows since $\pi$ is the law of $V:=\sum_{k=1}^{\infty} M_{1} \cdots M_{k-1} Q_{k-1} \neq 0$ w.p.1. Next, observe by Lemma 5.1 that

$$
\begin{aligned}
\int \mathbb{E}_{x}^{\alpha} & {\left[\sum_{i=0}^{\infty} F\left(X_{i}, S_{i}+s\right)\right] \varrho(d x, d s) } \\
& =\frac{1}{\lambda^{\prime}(\alpha)} \int F(x, s) \eta_{\alpha}(d x) d s=\frac{1}{\lambda^{\prime}(\alpha)} \int \frac{e^{-\alpha s}}{r_{\alpha}(x)} f\left(e^{s} x\right) \eta_{\alpha}(d x) d s \\
& =\frac{1}{\lambda^{\prime}(\alpha)} \int e^{-\alpha s} f\left(e^{s} x\right) l_{\alpha}(d x) d s,
\end{aligned}
$$

using that $\eta_{\alpha}(d x)=r_{\alpha}(x) l_{\alpha}(d x)$ [cf. (2.3) and the discussion there]. 
Also, by applying Lemma 3.6(ii), we obtain that

$$
\int_{\mathbb{D}} C(v) \pi(d v):=\int_{\mathbb{D}} r_{\alpha}(\widetilde{v}) \mathbb{E}_{\delta_{v}}^{\alpha}\left[|Z|^{\alpha} \mathbf{1}_{\{\tau=\infty\}}\right] \pi(d v)=C,
$$

where $C$ is given as in (2.10). Then (5.4), (5.5) and (5.6) imply that

$$
\begin{aligned}
\lim _{u \rightarrow \infty} u^{\alpha} \mathbb{E}\left[f\left(\frac{V}{u}\right)\right] & =\lim _{u \rightarrow \infty} u^{\alpha} \mathbb{E}\left[\hat{f}\left(\frac{V}{u}\right) \mathbf{1}_{\{|V| \geq u\}}\right] \\
& =\frac{C}{\lambda^{\prime}(\alpha)} \int_{\mathbb{S}_{+}^{d-1} \times \mathbb{R}_{+}} e^{-\alpha s} f\left(e^{s} x\right) l_{\alpha}(d x) d s
\end{aligned}
$$

for any bounded, almost $\theta$-Hölder continuous function $f$ satisfying the representation $f(x)=\hat{f}(x) \mathbf{1}_{\{|x| \geq 1\}}$, where $\hat{f}$ is $\theta$-Hölder continuous.

The validity of (5.1) for $f$ with $f(x)=\hat{f}(x) \mathbf{1}_{\{|x| \geq r\}}$, for general $r>0$, then follows by applying (5.7) to the function $\hat{f}_{r}(x)=r^{-\alpha} f(r v)$.

STEP 2. It remains to remove the assumption that $f$ is almost $\theta$-Hölder continuous, needed to apply Proposition 4.5 in the above argument. To this end, observe that for all $r>0$,

$$
\Upsilon_{u}^{(r)}:=u^{\alpha} \mathbb{P}\left(\frac{V}{u} \in \cdot \frac{|V|}{u} \geq r\right)
$$

defines a family of uniformly bounded measures on $\mathbb{R}_{+}^{d} \backslash B_{r}^{+}(0)$, where the boundedness follows by employing (5.1) with $f(x)=\mathbf{1}_{\{|x| \geq r\}}$, which is an almost $\theta$ Hölder continuous function. The Fourier characters $x \mapsto e^{i\langle x, y\rangle}$ are bounded Lipschitz continuous functions for any $y \in \mathbb{R}^{d}$; then $f_{y}(x):=e^{i\langle x, y\rangle} \mathbf{1}_{\{|x| \geq r\}}$ is almost $\theta$-Hölder continuous for any $\theta \leq \min \{1, \alpha\}$. Let $\mathfrak{L}_{\alpha}$ be the measure on $\mathbb{R}_{+}^{d} \backslash\{0\}$ defined by the equation

$$
\int_{\mathbb{S}_{+}^{d-1} \times \mathbb{R}} e^{-\alpha s} f\left(e^{s} x\right) l_{\alpha}(d x) d s=\int_{\mathbb{R}_{+}^{d} \backslash\{0\}} f(x) \mathfrak{L}_{\alpha}(d x),
$$

and let $\mathfrak{L}_{\alpha}^{(r)}$ denote its restriction to a measure on $\mathbb{R}_{+}^{d} \backslash B_{r}^{+}(0)$. Then, based on what we have proved so far, by considering real and imaginary parts separately we may infer the convergence, as $u \rightarrow \infty$, of

$$
\int e^{i\langle x, y\rangle} \Upsilon_{u}^{(r)}(d x)=u^{\alpha} \mathbb{E}\left[e^{i\left\langle u^{-1} V, y\right\rangle} \mathbf{1}_{\{|V| \geq r u\}}\right] \rightarrow \frac{C}{\lambda^{\prime}(\alpha)} \int e^{i\langle x, y\rangle} \mathfrak{L}_{\alpha}^{(r)}(d x),
$$

for all $y \in \mathbb{R}^{d}$. Then the Lévy continuity theorem yields the weak convergence $\Upsilon_{u}^{(r)} \Rightarrow \frac{C}{\lambda^{\prime}(\alpha)} \mathfrak{L}_{\alpha}^{(r)}$, for any $r>0$. Now if $f \in \mathscr{C}_{0}\left(\mathbb{R}_{+}^{d} \backslash\{0\}\right)$, then there exists $r>0$ such that $f$ is supported on $\left(B_{r}^{+}(0)\right)^{c}$. Hence

$$
\lim _{u \rightarrow \infty} u^{\alpha} \mathbb{E}\left[f\left(\frac{V}{u}\right)\right]=\lim _{u \rightarrow \infty} \int f(x) \Upsilon_{u}^{(r)}(d x)=\frac{C}{\lambda^{\prime}(\alpha)} \int f(x) \mathfrak{L}_{\alpha}(d x),
$$

that is, (5.1) holds. Finally, the equivalence of (5.1) to (5.2) follows from Theorem 2 in [43]. 
5.1. Smoothing. To remove Hypothesis $\left(\mathrm{H}_{3}\right)$, we employ a lower and upper approximation, where the approximating sequences are smoothed so that $\left(\mathrm{H}_{3}\right)$ is satisfied by these sequences.

We begin by constructing the lower approximating sequence. First, recall the condition $(\mathfrak{K})$ introduced just prior to the statement of Theorem 2.4. Also, from this discussion in Section 2, recall the definitions

$$
\widehat{M}_{n}:=M_{k n} \cdots M_{k(n-1)+1}, \quad \widehat{Q}_{n}:=\sum_{i=k(n-1)+1}^{k n} M_{k n} \cdots M_{i+1} Q_{i},
$$

for all $n \in \mathbb{N}_{+}$. Now let $k \in \mathbb{N}_{+}$be chosen such that $(\mathfrak{K})$ holds. Then $\left\{\left(\widehat{M}_{n}, \widehat{Q}_{n}\right)\right\}$ is an i.i.d. sequence under $\mathbb{P}$, and with positive probability, $\widehat{Q}_{1}-s \overrightarrow{1} \succ 0$ for some $s>0$. Let $\mathbb{B}_{n}=\left\{\widehat{Q}_{n}-s \overrightarrow{1} \succ 0\right\}$, and let $\chi_{n, \varepsilon}:=(-\varepsilon) \chi_{n}$ for an i.i.d. sequence $\left\{\chi_{n}\right\}$, independent of $\left\{\left(\widehat{M}_{n}, \widehat{Q}_{n}\right)\right\}$, such that $\chi_{1}$ has a nondegenerate absolutely continuous distribution concentrated on $[0,1]^{d}$ (and thus $\chi_{1, \varepsilon}$ is concentrated on $\left.[-\varepsilon, 0]^{d}\right)$.

For each $n$, set $\widehat{Q}_{n, \varepsilon}:=\widehat{Q}_{n}+\mathbf{1}_{\mathbb{B}_{n}} \chi_{n, \varepsilon}$ and note that, conditioned on the event $\mathbb{B}_{n}$, $\widehat{Q}_{n, \varepsilon}$ has a continuous distribution function. Then, since the event $\mathbb{B}_{n}$ occurs with positive probability, the distribution function of $\widehat{Q}_{n, \varepsilon}$ has an absolutely continuous component with respect to Lebesgue measure.

Now set

$$
V_{n, \varepsilon}=\widehat{M}_{n} V_{n-1, \varepsilon}+\widehat{Q}_{n, \varepsilon}, \quad n=1,2, \ldots ; \quad V_{0, \varepsilon}=V_{0} .
$$

Then $\left\{V_{n, \varepsilon}\right\}$ forms the smoothed lower sequence. Let

$$
V_{\varepsilon}:=\widehat{Q}_{1, \varepsilon}+\sum_{k=2}^{\infty} \widehat{M}_{1} \cdots \widehat{M}_{k-1} \widehat{Q}_{k, \varepsilon},
$$

and note that the law of $V_{\varepsilon}$ is the stationary distribution of the process $\left\{V_{n, \varepsilon}\right\}$, which we denote by $\pi_{\varepsilon}$.

A smoothed upper sequence is constructed analogously, now choosing $\chi_{n}^{\varepsilon}:=$ $\varepsilon \chi_{n}$, so that this random variable is concentrated on the interval $[0, \varepsilon]^{d}$. For each $n$, let $\widehat{Q}_{n}^{\varepsilon}=\widehat{Q}_{n}+\mathbf{1}_{\mathbb{B}_{n}} \chi_{n}^{\varepsilon}$. Then set $V_{0}^{\varepsilon}=V_{0}$ and

$$
V_{n}^{\varepsilon}=\widehat{M}_{n} V_{n-1}^{\varepsilon}+\widehat{Q}_{n}^{\varepsilon}, \quad n=1,2, \ldots ; \quad V^{\varepsilon}=\widehat{Q}_{1}^{\varepsilon}+\sum_{k=2}^{\infty} \widehat{M}_{1} \cdots \widehat{M}_{k-1} \widehat{Q}_{k}^{\varepsilon} .
$$

Let $\pi^{\varepsilon}$ denote the distribution of $V^{\varepsilon}$.

REMARK 5.3. At this stage, it should be emphasized that this smoothing construction only affects the random quantity $\widehat{Q}_{n}$, and not $\widehat{M}_{n}$, and so the function $\Lambda$ is unchanged. Thus, in particular, the solution $\alpha$ to the equation $\Lambda(\alpha)=0$ and the corresponding invariant function $r_{\alpha}$ and invariant measure $l_{\alpha}$ are the same as for the unsmoothed process. Moreover, since $\widehat{M}_{1}=M_{k} \cdots M_{1}$ and $\lambda(\alpha)=1$, the factor $\lambda^{\prime}(\alpha)$ must now be replaced with $k \lambda^{\prime}(\alpha)$; cf. Lemma 2.3. 
REMARK 5.4. Observe that if $k>1$ in $(\mathfrak{K})$, then the evolution of the lower and upper smoothed sequences cannot be compared to the dynamics of the process $\left\{V_{n}\right\}$, but to that of the $k$-step chain $\left\{V_{k n}: n \in \mathbb{N}\right\}$, which at time $n$ is equal to

$$
\widehat{V}_{n}:=\widehat{M}_{n} \cdots \widehat{M}_{1} V_{0}+\sum_{i=1}^{n} \widehat{M}_{n} \cdots \widehat{M}_{i+1} \widehat{Q}_{i}
$$

We then have the sandwich inequality

$$
V_{n, \varepsilon} \leq \widehat{V}_{n} \leq V_{n}^{\varepsilon} \quad \text { where } \widehat{V}_{n}=V_{k n} .
$$

For the remainder of this section, we consider the $k$-step chain $\left\{\widehat{V}_{n}\right\}$, defined in terms of $\left\{\left(\widehat{M}_{i}, \widehat{Q}_{i}\right)\right\}$. This $k$-step chain has the same stationary law, but different dynamics, compared with the 1-step chain $\left\{V_{n}\right\}$.

For any $x \in \mathbb{S}_{+}^{d-1}$ and $F \subset \mathbb{S}_{+}^{d-1}$, let $d(x, F)=\inf \{|x-y|: y \in F\}$; and for a given set $E \subset \mathbb{S}_{+}^{d-1}$, let

$$
E^{\varepsilon}=\left\{x \in \mathbb{S}_{+}^{d-1}: d(x, E) \leq \frac{2 \varepsilon}{s}\right\} \quad \text { and } \quad E_{\varepsilon}=\left\{x \in \mathbb{S}_{+}^{d-1}: d\left(x, E^{c}\right)>\frac{2 \varepsilon}{s}\right\} .
$$

LEMMA 5.5. Let $\varepsilon>0$. Then under the assumptions of Theorem 2.4:

(i) The approximating sequences $\left\{V_{n, \varepsilon}\right\}_{n \in \mathbb{N}}$ and $\left\{V_{n}^{\varepsilon}\right\}_{n \in \mathbb{N}}$ each satisfy Hypothesis $\left(\mathrm{H}_{3}\right)$.

(ii) For all $u>0$, we have the sandwich inequality

$$
\mathbb{P}\left(\left|V_{\varepsilon}\right|>u, \frac{V_{\varepsilon}}{\left|V_{\varepsilon}\right|} \in E_{\varepsilon}\right) \leq \mathbb{P}\left(|V|>u, \frac{V}{|V|} \in E\right) \leq \mathbb{P}\left(\left|V^{\varepsilon}\right|>u, \frac{V^{\varepsilon}}{\left|V^{\varepsilon}\right|} \in E^{\varepsilon}\right) .
$$

PROOF. (i) To verify part (i) of $\left(\mathrm{H}_{3}\right)$, let $P_{\varepsilon}$ denote the transition kernel of the process $\left\{V_{n, \varepsilon}\right\}$ in (5.9). Recall that $\chi_{\varepsilon}$ is independent of $\widehat{M}$ and $\widehat{Q}$. Hence, by construction,

$$
\begin{aligned}
P_{\varepsilon}(v, E) & =\mathbb{P}\left(\widehat{M} v+\widehat{Q} \in E, \mathbb{B}^{c}\right)+\int_{[-\varepsilon, 0]^{d}} \mathbb{P}(\widehat{M} v+\widehat{Q}+y \in E, \mathbb{B}) \mathbb{P}\left(\chi_{\varepsilon} \in d y\right) \\
& :=P_{1, \varepsilon}(v, E)+P_{2, \varepsilon}(v, E) .
\end{aligned}
$$

The kernel $P_{2, \varepsilon}$ is obtained by the convolution of $\mathbb{P}(\widehat{M} v+\widehat{Q} \in \cdot, \mathbb{B})$ with the probability measure $\mathbb{P}\left(\chi_{\varepsilon} \in \cdot\right)$, which, by assumption, is smooth; thus $P_{2, \varepsilon}(v, \cdot)$ itself has a Lebesgue density for all $v \in \mathbb{R}_{+}^{d}$. Hence part (i) of $\left(\mathrm{H}_{3}\right)$ is satisfied with $\Phi$ taken to be Lebesgue measure and $F=\mathbb{R}_{+}^{d}$.

Since $\pi_{\varepsilon}$ is the stationary distribution of the Markov chain with transition kernel $P_{\varepsilon}$, it follows that $\pi_{\varepsilon}$ has a continuous component with respect to Lebesgue measure. Hence, $\left(\operatorname{supp} \pi_{\varepsilon}\right)^{\circ} \neq \varnothing$ and part (ii) of $\left(\mathrm{H}_{3}\right)$ is satisfied.

The verification for the process $\left\{V_{n}^{\varepsilon}\right\}$ is analogous. 
(ii) By construction,

$$
V-V_{\varepsilon}=-\mathbf{1}_{\mathbb{B}_{1}} \chi_{1, \varepsilon}-\sum_{k=1}^{\infty} \widehat{M}_{1} \cdots \widehat{M}_{k} \mathbf{1}_{\mathbb{B}_{k+1}} \chi_{k+1, \varepsilon},
$$

since $\widehat{Q}_{k}-\widehat{Q}_{k, \varepsilon}=\mathbf{1}_{\mathbb{B}_{k}} \chi_{\varepsilon}$. [Here, we define $\mathbb{B}_{k}$ in the same way as $\mathbb{B}$, but with respect to the pair $\left(\widehat{M}_{k}, \widehat{Q}_{k}\right)$.] Consequently, setting $\widehat{M}_{0}$ to be equal to the identity matrix and recalling that $\chi_{\varepsilon}$ is supported on $[-\varepsilon, 0]^{d}$, we obtain that

$$
\left|V-V_{\varepsilon}\right| \leq \varepsilon\left|\sum_{k=0}^{\infty}\left(\widehat{M}_{0} \cdots \widehat{M}_{k} \overrightarrow{1}\right) \mathbf{1}_{\mathbb{B}_{k+1}}\right|
$$

Moreover,

$$
|V|=\left|\sum_{k=0}^{\infty} \widehat{M}_{0} \cdots \widehat{M}_{k} \widehat{Q}_{k+1}\right| \geq s\left|\sum_{k=0}^{\infty}\left(\widehat{M}_{0} \cdots \widehat{M}_{k} \overrightarrow{1}\right) \mathbf{1}_{\mathbb{B}_{k+1}}\right| .
$$

This implies that

$$
\left|\frac{V}{|V|}-\frac{V^{\varepsilon}}{\left|V^{\varepsilon}\right|}\right| \leq \frac{1}{|V|}\left|V-V^{\varepsilon}\right|+\left|V^{\varepsilon}\right|\left|\frac{1}{|V|}-\frac{1}{\left|V^{\varepsilon}\right|}\right| \leq 2 \frac{\left|V-V^{\varepsilon}\right|}{|V|} \leq \frac{2 \varepsilon}{s} .
$$

Hence,

$$
\frac{V_{\varepsilon}}{\left|V_{\varepsilon}\right|} \in E_{\varepsilon} \Rightarrow d\left(\frac{V_{\varepsilon}}{\left|V_{\varepsilon}\right|}, E^{c}\right)>\frac{2 \varepsilon}{s} \Rightarrow d\left(\frac{V}{|V|}, E^{c}\right)>0 \Rightarrow \frac{V}{|V|} \in E .
$$

Furthermore, by (5.11), we also have that $\left|V_{\varepsilon}\right|>u \Rightarrow|V|>u$. Consequently,

$$
\mathbb{P}\left(\left|V_{\varepsilon}\right|>u, \frac{V_{\varepsilon}}{\left|V_{\varepsilon}\right|} \in E_{\varepsilon}\right) \leq \mathbb{P}\left(|V|>u, \frac{V}{|V|} \in E\right) .
$$

The remaining inequality of part (ii) is established analogously.

Since Hypothesis $\left(\mathrm{H}_{3}\right)$ is satisfied for the two approximating sequences in Lemma 5.5, it is natural to apply Proposition 5.2 to these sequences, yielding upper and lower bounds for $\mathbb{P}(|V|>u, V /|V| \in E)$ as $u \rightarrow \infty$.

Let $\left|\widehat{Z}_{\varepsilon}\right|$ be defined the same as the random variable $|Z|$ in Section 3.2, but with respect to the process $\left\{\left(\widehat{M}_{i}, \widehat{Q}_{i, \varepsilon}\right): i=1,2, \ldots\right\}$; namely,

$$
\left|\widehat{Z}_{\varepsilon}\right|=|v|+\sum_{i=1}^{\infty} \frac{\left\langle\widehat{Y}_{i},\left(\widehat{Q}_{i, \varepsilon}\right)^{\sim}\right\rangle}{\left\langle\widehat{Y}_{i}, \widehat{X}_{i}\right\rangle} \frac{\left|\widehat{Q}_{i, \varepsilon}\right|}{\left|\widehat{M}_{i} \cdots \widehat{M}_{1} \widetilde{v}\right|} \quad \mathbb{P}_{\delta_{v}}^{\alpha} \text {-a.s. }
$$

where

$$
\widehat{Y}_{i}:=\lim _{n \rightarrow \infty}\left(\widehat{M}_{i}^{\top} \cdots \widehat{M}_{n}^{\top} \overrightarrow{1}\right)^{\sim}, \quad n=1,2, \ldots
$$


and $\widehat{X}_{i}=\left(\widehat{M}_{i} \cdots \widehat{M}_{1} v\right)^{\sim}$. Then, with $E=\mathbb{S}_{+}^{d-1}$, we obtain by Proposition 5.2, Lemma 5.5, and Remark 5.3 that

$$
\frac{C_{\varepsilon}}{\alpha k \lambda^{\prime}(\alpha)} \leq u^{\alpha} \liminf _{u \rightarrow \infty} \mathbb{P}(|V|>u) \leq \limsup _{u \rightarrow \infty} u^{\alpha} \mathbb{P}(|V|>u) \leq \frac{C^{\varepsilon}}{\alpha k \lambda^{\prime}(\alpha)},
$$

where, in view of Lemma 3.6(ii), we have

$$
C_{\varepsilon}=\int_{\mathbb{D}} r_{\alpha}(\widetilde{v}) \mathbb{E}_{\delta_{v}}^{\alpha}\left[\left|\widehat{Z}_{\varepsilon}\right|^{\alpha} \mathbf{1}_{\left\{\tau_{\varepsilon}=\infty\right\}}\right] \pi_{\varepsilon}(d v)
$$

and

$$
C^{\varepsilon}=\int_{\mathbb{D}} r_{\alpha}(\widetilde{v}) \mathbb{E}_{\delta_{v}}^{\alpha}\left[\left|\widehat{Z}^{\varepsilon}\right|^{\alpha} \mathbf{1}_{\left\{\tau^{\varepsilon}=\infty\right\}}\right] \pi^{\varepsilon}(d v) .
$$

In what follows, we will generally write $\tau \equiv \tau(\mathbb{D})$ to emphasize the dependence of this quantity on the choice of $\mathbb{D}$. However, it is important to observe from Proposition 5.2 that $C_{\varepsilon}$ and $C^{\varepsilon}$ are universal constants, not dependent on the choice of $\mathbb{D}$.

The next lemma shows that these constants converge to the required constant $C$ in $(2.10)$ as $\varepsilon \downarrow 0$.

Lemma 5.6. Assume the conditions of Theorem 2.4. Then for any set $\mathbb{D}=$ $B_{r}^{+}(0)$ with $\pi(\mathbb{D})>0$,

$$
C_{\varepsilon} \nearrow C \text { and } C^{\varepsilon} \searrow C \text { as } \varepsilon \rightarrow 0 \text {, }
$$

where $C$ is independent of the choice of $\mathbb{D}$ and has the representation

$$
C=\int_{\mathbb{D}} r_{\alpha}(\widetilde{v}) \mathbb{E}_{\delta_{v}}^{\alpha}\left[|\widehat{Z}|^{\alpha} \mathbf{1}_{\{\tau(\mathbb{D})=\infty\}}\right] \pi(d v) .
$$

PROOF. To establish the result, we will show

$$
\lim _{\varepsilon \rightarrow 0} C_{\varepsilon} \geq C(\mathbb{D}) \quad \text { and } \quad \lim _{\varepsilon \rightarrow 0} C^{\varepsilon} \leq C(\mathbb{D}),
$$

where $C(\mathbb{D})$ represents the quantity on the right-hand side of (5.18). Note that these limits necessarily exist, since $C_{\varepsilon},-C^{\varepsilon}$ are monotonically increasing [as follows from the monotonicity, in $\varepsilon$, of $V_{\varepsilon}$ and $V^{\varepsilon}$ and (5.2)]. Then (5.19) yields $\lim _{\varepsilon \rightarrow 0} C_{\varepsilon}=C(\mathbb{D})=\lim _{\varepsilon \rightarrow 0} C^{\varepsilon}$.

STEP 1 . We begin by establishing that $\lim _{\varepsilon \rightarrow 0} C_{\varepsilon} \geq C(\overline{\mathbb{D}})$. Set

$$
H_{\varepsilon}(v)=r_{\alpha}(v) \mathbb{E}_{\delta_{v}}^{\alpha}\left[\left|\widehat{Z}_{\varepsilon}\right|^{\alpha} \mathbf{1}_{\left\{\tau_{\varepsilon}(\overline{\mathbb{D}})=\infty\right\}}\right] ; \quad H(v)=r_{\alpha}(v) \mathbb{E}_{\delta_{v}}^{\alpha}\left[|\widehat{Z}|^{\alpha} \mathbf{1}_{\{\tau(\overline{\mathbb{D}})=\infty\}}\right] .
$$

Then we need to show that

$$
\liminf _{\varepsilon \rightarrow 0} \int_{\overline{\mathbb{D}}} H_{\varepsilon}(v) \pi_{\varepsilon}(d v) \geq \int_{\overline{\mathbb{D}}} H(v) \pi(d v) .
$$

We will prove below that: (i) $H_{\varepsilon}(v) \uparrow H(v)$ as $\varepsilon \rightarrow 0$; and (ii) for all $\varepsilon \geq 0$, the function $v \mapsto H_{\varepsilon}(v)$ is lower semicontinuous. 
Assume that (i) and (ii) hold, and fix $\varepsilon_{0}>0$. Since $H_{\varepsilon}(v)$ is a monotone increasing sequence as $\varepsilon \downarrow 0$,

$$
C_{\varepsilon} \geq \int_{\overline{\mathbb{D}}} H_{\varepsilon_{0}}(v) \pi_{\varepsilon}(d v) \quad \text { for all } \varepsilon \leq \varepsilon_{0} .
$$

As the function $v \mapsto H_{\varepsilon_{0}}(v)$ is lower semicontinuous and bounded from below by 0 , and $\pi_{\varepsilon} \Rightarrow \pi$ [cf. (5.11)], in then follows from the Portmanteau theorem ([49], Theorem 1.3.4(iv)) that

$$
\liminf _{\varepsilon \rightarrow 0} C_{\varepsilon} \geq \liminf _{\varepsilon \rightarrow 0} \int_{\overline{\mathbb{D}}} H_{\varepsilon_{0}}(v) \pi_{\varepsilon}(d v) \geq \int_{\overline{\mathbb{D}}} H_{\varepsilon_{0}}(v) \pi(d v) .
$$

Now let $\varepsilon_{0} \rightarrow 0$ and use the monotone convergence $H_{\varepsilon_{0}} \uparrow H$ to infer by the monotone convergence theorem that

$$
\liminf _{\varepsilon \rightarrow 0} C_{\varepsilon} \geq \lim _{\varepsilon_{0} \rightarrow 0} \int_{\overline{\mathbb{D}}} H_{\varepsilon_{0}}(v) \pi(d v)=\int_{\overline{\mathbb{D}}} H(v) \pi(d v)=C(\overline{\mathbb{D}}) .
$$

It remains to prove (i) and (ii). In order to obtain (i), observe that $\left|V_{n, \varepsilon}\right|$ increases monotonically to $\left|\widehat{V}_{n}\right|$ as $\varepsilon \rightarrow 0$. Thus, if the process $\left\{\widehat{V}_{n}\right\}$ enters $\overline{\mathbb{D}}$, then so does $\left\{V_{n, \varepsilon}\right\}$, for all $\varepsilon>0$. Hence, we trivially obtain that $\mathbf{1}_{\{\tau(\overline{\mathbb{D}})=\infty\}} \geq \mathbf{1}_{\left\{\tau_{\varepsilon}(\overline{\mathbb{D}})=\infty\right\}}$, where $\tau(\overline{\mathbb{D}}), \tau_{\varepsilon}(\overline{\mathbb{D}})$ are the first passage times of $\left\{\widehat{V}_{n}\right\},\left\{V_{n, \varepsilon}\right\}$ into $\overline{\mathbb{D}}$, respectively. Conversely, observe that if $\tau(\overline{\mathbb{D}})=\infty$, then $\mathbf{V}:=\left(\widehat{V}_{1}, \widehat{V}_{2}, \ldots\right) \in\left(\overline{\mathbb{D}}^{c}\right)^{\mathbb{N}}$, which is open. Now $\mathbf{V}_{\varepsilon}:=\left(V_{1, \varepsilon}, V_{2, \varepsilon}, \ldots\right)$ converges to $\mathbf{V}$ a.s. in the product topology (as $\chi_{\varepsilon}$ is supported on $[-\varepsilon, 0]^{d}$ ). It follows that $\mathbf{V}_{\varepsilon} \in\left(\overline{\mathbb{D}}^{c}\right)^{\mathbb{N}}$ for sufficiently small $\varepsilon$. Consequently, $\mathbf{1}_{\{\tau(\overline{\mathbb{D}})=\infty\}} \leq \liminf _{\varepsilon \rightarrow 0} \mathbf{1}_{\left\{\tau_{\varepsilon}(\overline{\mathbb{D}})=\infty\right\}}$. Thus we conclude that $\mathbf{1}_{\{\tau(\overline{\mathbb{D}})=\infty\}}=\lim _{\varepsilon \rightarrow 0} \mathbf{1}_{\left\{\tau_{\varepsilon}(\overline{\mathbb{D}})=\infty\right\}}$ and, moreover, the convergence is monotone, that is, $\mathbf{1}_{\left\{\tau_{\varepsilon}(\overline{\mathbb{D}})=\infty\right\}} \uparrow \mathbf{1}_{\{\tau(\overline{\mathbb{D}})=\infty\}}$ as $\varepsilon \rightarrow 0$. Furthermore, as $\widehat{Q}_{\varepsilon}$ increases componentwise to $\widehat{Q}$, we deduce from (5.16) and Lemma 3.6(ii) that $\left|\widehat{Z}_{\varepsilon}\right| \uparrow|\widehat{Z}|$ as $\varepsilon \rightarrow 0$. Also, by Lemma 3.6(iii), $|\widehat{Z}|^{\alpha} \mathbf{1}_{\{\tau(\overline{\mathbb{D}})=\infty\}}$ is an integrable upper bound for the family $\left\{\left|\widehat{Z}_{\varepsilon}\right|^{\alpha} \mathbf{1}_{\left\{\tau_{\varepsilon}(\overline{\mathbb{D}})=\infty\right\}}\right\}_{\varepsilon>0}$, and thus we obtain the monotone convergence $H_{\varepsilon}(v) \uparrow$ $H(v)$ as $\varepsilon \rightarrow 0$, for all $v \in \overline{\mathbb{D}}$.

To obtain (ii), observe that if $v \rightarrow \hat{v}$, then $\mathbf{V}(v)$ converges to $\mathbf{V}(\hat{v})$, where $\mathbf{V}:=\left(V_{1, \varepsilon}, V_{2, \varepsilon}, \ldots\right)$, and by writing $\mathbf{V}(v)$, we emphasize the dependence of this quantity on its initial state. Then by repeating the argument given above, we obtain that $\overline{\mathbb{D}}$ closed $\Rightarrow \mathbf{1}_{\{\tau(\overline{\mathbb{D}}, \hat{v})=\infty\}} \leq \liminf _{v \rightarrow \hat{v}} \mathbf{1}_{\{\tau(\overline{\mathbb{D}}, \hat{v})=\infty\}}$, where, once again, $\tau(\overline{\mathbb{D}}, \cdot)$ denotes the dependence on the initial state. From the representation (5.16), we deduce that the function $v \mapsto \widehat{Z}_{\varepsilon}(v)$ is continuous a.s. [namely, this series converges a.s. by Lemma 3.6(i)]. Then we may apply Fatou's lemma and use the continuity of $r_{\alpha}$ to infer that $H_{\varepsilon}$ is lower semicontinuous.

STEP 2. To establish the second inequality in (5.19), we proceed as before, now using $\left(\mathrm{i}^{\prime}\right)$ the convergence

$$
H^{\varepsilon}(v):=r_{\alpha}(v) \mathbb{E}_{\boldsymbol{\delta}_{v}}^{\alpha}\left[\left|\widehat{Z}^{\varepsilon}\right|^{\alpha} \mathbf{1}_{\left\{\tau^{\varepsilon}(\mathbb{D})=\infty\right\}}\right] \searrow r_{\alpha}(v) \mathbb{E}_{\boldsymbol{\delta}_{v}}^{\alpha}\left[|\widehat{Z}|^{\alpha} \mathbf{1}_{\{\tau(\mathbb{D})=\infty\}}\right]:=H^{\circ}(v)
$$


and (ii') the upper semicontinuity of $H^{\varepsilon}(v)$, which follows since we consider now the hitting time of an open set. Furthermore, Lemma 3.6(iii) yields that $\sup _{v \in \mathbb{D}} H_{\varepsilon_{0}}(v) \leq B$, for some finite constant $B$. Then we may apply the Portmanteau theorem ([49], Theorem 1.3.4(v)) to infer that

$$
\limsup _{\varepsilon \rightarrow 0} C^{\varepsilon} \leq \limsup _{\varepsilon \rightarrow 0} \int_{\mathbb{D}} H^{\varepsilon_{0}}(v) \pi^{\varepsilon}(d v)=\int_{\mathbb{D}} H^{\varepsilon_{0}}(v) \pi(d v),
$$

for all $\varepsilon_{0}>0$, and thus, letting $\varepsilon_{0} \rightarrow 0$ and using (i'),

$$
\limsup _{\varepsilon \rightarrow 0} C^{\varepsilon} \leq \int_{\mathbb{D}} H^{\circ}(v) \pi(d v)=C(\mathbb{D}) .
$$

STEP 3. It remains to show that if $\mathbb{D}=B_{r}^{+}(0)$, where $\pi(\mathbb{D})>0$, then, in the first equation in (5.19), we in fact have that $\lim _{\varepsilon \rightarrow 0} C_{\varepsilon} \geq C(\mathbb{D})$. To this end, let $\left\{r_{i}\right\}$ be chosen such that $r_{i} \uparrow r$ as $i \rightarrow \infty$, and set $\mathbb{D}_{i}=B_{r_{i}}^{+}(0)$. If $\left\{\widehat{V}_{n}\right\}$ avoids $\mathbb{D}$, then it also avoids each $\mathbb{D}_{i}$, so we trivially obtain that $\mathbf{1}_{\left\{\tau\left(\overline{\mathbb{D}}_{i}\right)=\infty\right\}} \geq \mathbf{1}_{\{\tau(\mathbb{D})=\infty\}}$. Conversely, $V_{n} \in \mathbb{D} \Rightarrow V_{n} \in \overline{\mathbb{D}}_{i}$ for sufficiently large $i$. Thus, $\lim _{i \rightarrow \infty} \mathbf{1}_{\left\{\tau\left(\overline{\mathbb{D}}_{i}\right)=\infty\right\}}=$ $\mathbf{1}_{\{\tau(\mathbb{D})=\infty\}}$. Now $\lim _{\varepsilon \rightarrow 0} C_{\varepsilon}$ is a universal constant, independent of the choice of the set $\overline{\mathbb{D}}$ in (5.19). Consequently, we conclude by (5.19) that

$$
\begin{aligned}
\lim _{\varepsilon \rightarrow 0} C_{\varepsilon} & \geq \lim _{i \rightarrow \infty} C\left(\overline{\mathbb{D}}_{i}\right)=\lim _{i \rightarrow \infty} \int_{\overline{\mathbb{D}}_{i}} r_{\alpha}(\widetilde{v}) \mathbb{E}_{\delta_{v}}^{\alpha}\left[|\widehat{Z}|^{\alpha} \mathbf{1}_{\left\{\tau\left(\overline{\mathbb{D}}_{i}\right)=\infty\right\}}\right] \pi(d v) \\
& =\int_{\mathbb{D}} r_{\alpha}(\widetilde{v}) \mathbb{E}_{\delta_{v}}^{\alpha}\left[|\widehat{Z}|^{\alpha} \mathbf{1}_{\{\tau(\mathbb{D})=\infty\}] \pi(d v)=C(\mathbb{D})}\right.
\end{aligned}
$$

as required.

Proof of Theorem 2.4. It follows directly from Proposition 5.2 and Lemmas 5.5 and 5.6 that for any $E \in \mathscr{B}\left(\mathbb{S}_{+}^{d-1}\right)$,

$$
\liminf _{u \rightarrow \infty} u^{\alpha} \mathbb{P}\left(|V|>t u, \frac{V}{|V|} \in E\right) \geq \frac{C}{\alpha k \lambda^{\prime}(\alpha)} t^{-\alpha} \limsup _{\varepsilon \rightarrow 0} l_{\alpha}\left(E_{\varepsilon}\right)
$$

and

$$
\limsup _{u \rightarrow \infty} u^{\alpha} \mathbb{P}\left(|V|>t u, \frac{V}{|V|} \in E\right) \leq \frac{C}{\alpha k \lambda^{\prime}(\alpha)} t^{-\alpha} \liminf _{\varepsilon \rightarrow 0} l_{\alpha}\left(E^{\varepsilon}\right) .
$$

Now if $l_{\alpha}(\partial E)=0$, then

$$
\limsup _{\varepsilon \rightarrow 0} l_{\alpha}\left(E_{\varepsilon}\right)=\liminf _{\varepsilon \rightarrow 0} l_{\alpha}\left(E^{\varepsilon}\right)=l_{\alpha}(E) .
$$

Hence, the two bounds coincide, and thus, for all measurable $E \subset \mathbb{S}_{+}^{d-1}$ with $l_{\alpha}(\partial E)=0$,

$$
\lim _{u \rightarrow \infty} u^{\alpha} \mathbb{P}\left(|V|>t u, \frac{V}{|V|} \in E\right)=\frac{C}{\alpha k \lambda^{\prime}(\alpha)} t^{-\alpha} l_{\alpha}(E) .
$$


By the Portmanteau theorem, this implies the weak convergence

$$
u^{\alpha} \mathbb{P}\left(|V|>t u, \frac{V}{|V|} \in \cdot\right) \Rightarrow \frac{C}{\alpha \lambda^{\prime}(\alpha)} t^{-\alpha} l_{\alpha}(\cdot) \quad \text { as } u \rightarrow \infty,
$$

for all $t>0$, which is equivalent to (2.9) by Theorem 2 of [43].

6. Proof of Theorem 2.8. Next, we turn to the proof of Theorem 2.8 . Throughout this section, assume that the set $A$ is a semi-cone.

We begin by identifying the constant appearing in the ruin problem for the random walk $\left\{\left(X_{n}, S_{n}^{A}\right)\right\}$. For this purpose, define

$$
\mathfrak{T}_{u}^{A}=\inf \left\{n \in \mathbb{N}: M_{n} \cdots M_{1} \widetilde{V}_{0} \in u A\right\} .
$$

LEMmA 6.1. Suppose that $\left(\mathrm{H}_{1}\right)$ and $\left(\mathrm{H}_{2}\right)$ are satisfied, and assume that $d_{A}$ is bounded and continuous on $\mathbb{S}_{+}^{d-1}$. Then for all $v \in \mathbb{R}_{+}^{d} \backslash\{0\}$,

$$
\lim _{u \rightarrow \infty} u^{\alpha} \mathbb{P}\left(\mathfrak{T}_{u}^{A}<\infty \mid V_{0}=v\right)=r_{\alpha}(\widetilde{v}) \int \frac{e^{-\alpha s}}{r_{\alpha}^{A}(x)} \varrho^{A}(d x, d s):=r_{\alpha}(\widetilde{v}) D_{A},
$$

where $\varrho^{A}$ is the asymptotic overjump distribution of the process $\left\{\left(X_{n}, S_{n}^{A}\right)\right\}$.

PROOF. Converting to the $\alpha$-shifted measure, we obtain that for any $v \in \mathbb{R}_{+}^{d} \backslash$ $\{0\}$,

$$
u^{\alpha} \mathbb{P}\left(\mathfrak{T}_{u}^{A}<\infty \mid V_{0}=v\right)=r_{\alpha}(\widetilde{v}) \mathbb{E}_{\tilde{v}}^{\alpha}\left[e^{-\alpha\left(S_{\mathfrak{T}_{u}^{A}}^{A}-\log u\right)}\left(r_{\alpha}^{A}\left(X_{\mathfrak{T}_{u}^{A}}\right)\right)^{-1} \mathbf{1}_{\left\{\mathfrak{T}_{u}^{A}<\infty\right\}}\right],
$$

using the definitions of $S_{n}^{A}$ and $r_{\alpha}^{A}$. To characterize the limit on the right-hand side, use the weak convergence (3.31), which holds due to Kesten's renewal theorem.

To establish the weak convergence of $\left\{T_{u}^{A} / u^{\alpha}\right\}$, the main idea will be to study the excursions of $\left\{V_{n}\right\}$ over cycles emanating from the set $\mathbb{D}$. For this purpose, we introduce the random variables

$$
U_{i}:=\max _{\kappa_{i-1}<n \leq \kappa_{i}} V_{n}^{A}, \quad i=1,2, \ldots,
$$

where $V_{n}^{A}:=V_{n} / d_{A}\left(\tilde{V}_{n}\right)$ and $\left\{\kappa_{i}\right\}$ denote the successive return times to $\mathbb{D}$; that is, $\kappa_{i}=\inf \left\{n>\kappa_{i-1}: V_{n} \in \mathbb{D}\right\}, i \in \mathbb{N}_{+} ; \kappa_{0}=0$. For $n \in \mathbb{N}_{+}$, also set

$$
\mathscr{M}_{n}^{U}=\max \left\{U_{1}, \ldots, U_{n}\right\} ; \quad \mathscr{M}_{n}=\max \left\{V_{1}^{A}, \ldots, V_{n}^{A}\right\} .
$$

Recall that $\left\{T_{u}^{A} \leq N\right\}=\left\{V_{n}^{A}>u\right.$, some $\left.n \leq N\right\}$. Thus, $\left\{\mathscr{M}_{n}^{U}>u\right\}$ describes the 
event that $T_{u}^{A}$ occurs by the random time $\kappa_{n}$, while $\left\{\mathscr{M}_{n}>u\right\}$ describes the event that $T_{u}^{A}$ occurs by the deterministic time $n$.

Proposition 6.2. Suppose that $\left(\mathrm{H}_{1}\right)$ and $\left(\mathrm{H}_{2}\right)$ are satisfied, and suppose that there exists $m \in \mathbb{N}_{+}$such that $\left(\mathrm{H}_{3}\right)$ holds for the $m$-skeleton $\left\{V_{m n}: n \in \mathbb{N}\right\}$. Assume that $\mathbb{D} \in \mathscr{B}\left(\mathbb{R}_{+}^{d}\right)$ is bounded and $\pi(\mathbb{D})>0$, and suppose that the function $d_{A}$ is bounded and continuous on $\mathbb{S}_{+}^{d-1}$. Then for all $v \in \mathbb{R}_{+}^{d} \backslash\{0\}$,

$$
\lim _{n \rightarrow \infty} \mathbb{P}\left(\mathscr{M}_{n}^{U} \leq n^{1 / \alpha} u \mid V_{0}=v\right)=\exp \left\{-K_{A} \mathbb{E}_{\pi_{\mathbb{D}}}[\tau] u^{-\alpha}\right\}
$$

where $K_{A}=C D_{A}$ and $C$ is given as in (2.10).

Unless explicitly noted, we assume throughout the rest of this section that $V_{0}=v$ for a fixed $v \in \mathbb{R}_{+}^{d} \backslash\{0\}$, that is, $\mathbb{P}=\mathbb{P}_{v}$.

ProOF. Set $u_{n}=n^{1 / \alpha} u$. Then for any $l \in \mathbb{N}_{+}$,

$$
\begin{aligned}
& \sum_{i=1}^{l} \mathbb{P}\left(U_{i}>u_{n}\right)-\sum_{1 \leq i<j \leq l} \mathbb{P}\left(U_{i}>u_{n}, U_{j}>u_{n}\right) \\
& \leq \mathbb{P}\left(\mathscr{M}_{l}^{U}>u_{n}\right) \leq \sum_{i=1}^{l} \mathbb{P}\left(U_{i}>u_{n}\right) .
\end{aligned}
$$

Now fix $k \in \mathbb{N}_{+}$. We begin by calculating $\sum_{i=1}^{l(n)} \mathbb{P}\left(U_{i}>u_{n}\right)$, as $n \rightarrow \infty$, for the sequence $l(n)=\lfloor n / k\rfloor$. By equation (4.2) of Corollary 4.2 and the Markov property, we have that for all $i \in \mathbb{N}_{+}$and $w \in \mathbb{R}_{+}^{d} \backslash\{0\}$,

$$
\begin{aligned}
\lim _{n \rightarrow \infty} & n u^{\alpha} \mathbb{P}\left(U_{i}>u_{n} \mid V_{\kappa_{i-1}}=w\right) \\
& =\lim _{n \rightarrow \infty} n u^{\alpha} \mathbb{P}\left(T_{u}^{A}<\tau \mid V_{0}=w\right) \\
& =r_{\alpha}(\widetilde{w}) \mathbb{E}_{\delta_{w}}^{\alpha}\left[|Z|^{\alpha} \mathbf{1}_{\{\tau=\infty\}}\right] \int \frac{e^{-\alpha s}}{r_{\alpha}^{A}(x)} \varrho^{A}(d x, d s) \\
& :=H(w)=C(w) D_{A} .
\end{aligned}
$$

Under Hypotheses $\left(\mathrm{H}_{3}\right),\left\{V_{m n}: n \geq 0\right\}$ is a positive aperiodic Harris chain (Lemma 3.4). Then $\left\{V_{n}\right\}$, and hence $\left\{V_{\kappa_{i}}\right\}$, are positive $m$-periodic Harris chains (cf. [1], Theorem 8.3.7), and the invariant measure of $\left\{V_{\kappa_{i}}\right\}$ is $\pi_{\mathbb{D}}$ (cf. Lemma 3.3). If $\gamma_{i}$ denotes the law of $V_{\kappa_{i}}, i \in \mathbb{N}_{+}$, then Harris recurrence gives that $\left|n^{-1} \sum_{i=1}^{n} \gamma_{i}-\pi_{\mathbb{D}}\right| \mathrm{TV} \rightarrow 0$ as $n \rightarrow \infty$, where $|\cdot| \mathrm{TV}$ denotes the total variation distance; see [37], Theorem 13.3.4. Set

$$
H_{n}(w)=n u^{\alpha} \mathbb{P}\left(U_{1}>u_{n} \mid V_{0}=w\right) .
$$


Using (4.17) with $h \equiv 1$, we have $\sup \left\{H_{n}(w): w \in \mathbb{D} \backslash\{0\}, n \in \mathbb{N}\right\} \leq B<\infty$. Now $n u^{\alpha} \mathbb{P}\left(U_{i}>u_{n}\right)=\int_{\mathbb{D}} H_{n}(w) \gamma_{i-1}(d w)$, and

$$
\begin{aligned}
\left|\frac{k}{n} \sum_{i=1}^{\lfloor n / k\rfloor} n u^{\alpha} \mathbb{P}\left(U_{i}>u_{n}\right)-\int_{\mathbb{D}} H(w) \pi_{\mathbb{D}}(d w)\right| \\
\leq \mid \int_{\mathbb{D}} H_{n}(w)\left(\frac{k}{n} \sum_{i=1}^{\lfloor n / k\rfloor} \gamma_{i-1}-\pi_{\mathbb{D}}\right)(d w) \\
\quad-\int_{\mathbb{D}}\left(H_{n}(w)-H(w)\right) \pi_{\mathbb{D}}(d w) \mid \\
\leq B\left|\frac{k}{n} \sum_{i=1}^{\lfloor n / k\rfloor} \gamma_{i-1}-\pi_{\mathbb{D}}\right|_{\mathrm{TV}}+\int_{\mathbb{D}}\left|H_{n}(w)-H(w)\right| \pi_{\mathbb{D}}(d w) .
\end{aligned}
$$

The second term tends to zero as $n \rightarrow \infty$ by dominated convergence and the fact that $H_{n}(w) \rightarrow H(w)$, by (6.4). Thus, the left-hand side of (6.5) tends to zero as $n \rightarrow \infty$, and hence, using (6.4),

$$
\begin{aligned}
\lim _{n \rightarrow \infty} \sum_{i=1}^{\lfloor n / k\rfloor} u^{\alpha} \mathbb{P}\left(U_{i}>u_{n}\right) & =\frac{1}{k} \int_{\mathbb{D}} H(w) \pi_{\mathbb{D}}(d w) \\
& =\frac{D_{A}}{k} \int_{\mathbb{D}} C(w) \frac{\pi(d w)}{\pi(\mathbb{D})}=\frac{K_{A} \mathbb{E}_{\pi_{\mathbb{D}}}[\tau]}{k},
\end{aligned}
$$

since $C=\int_{\mathbb{D}} C(w) \pi(d w)$ and $\mathbb{E}_{\pi_{\mathbb{D}}}[\tau]=(\pi(\mathbb{D}))^{-1}$ (by Lemma 3.3). Substituting this equation into (6.3), we deduce that for any $k \in \mathbb{N}_{+}$,

$$
\limsup _{n \rightarrow \infty} \mathbb{P}\left(\mathscr{M}_{\lfloor n / k\rfloor}^{U}>u_{n}\right) \leq \frac{K_{A} \mathbb{E}_{\pi_{\mathbb{D}}}[\tau]}{k} u^{-\alpha} .
$$

Note that the right-hand side is independent of $V_{0} \in \mathbb{R}_{+}^{d} \backslash\{0\}$, and hence the same calculation yields the asymptotic behavior of the maximum over any block of comparable length; in particular, for $\limsup _{n \rightarrow \infty} \mathbb{P}\left(U_{\lfloor j n / k\rfloor+1}, \ldots, U_{\lfloor(j+1) n / k\rfloor}>u_{n} \mid\right.$ $\left.\mathscr{F}_{\kappa_{\lfloor j n / k\rfloor}}\right), j=0, \ldots, k-1$. Hence, letting $k \rightarrow \infty$, we conclude by (6.7) that

$$
\limsup _{n \rightarrow \infty} \mathbb{P}\left(\mathscr{M}_{n}^{U} \leq u_{n}\right) \leq\left(1-\frac{K_{A} \mathbb{E}_{\pi_{\mathbb{D}}}[\tau] u^{-\alpha}}{k}\right)^{k} \rightarrow \exp \left\{-K_{A} \mathbb{E}_{\pi_{\mathbb{D}}}[\tau] u^{-\alpha}\right\}
$$

as $k \rightarrow \infty$. Moreover, again using the upper bound provided by Lemma 4.6 (uniform in the initial state), we obtain that for any positive integer $k$,

$$
\limsup _{n \rightarrow \infty} \sum_{1 \leq i<j \leq\lfloor n / k\rfloor} \mathbb{P}\left(U_{i}>u_{n}, U_{j}>u_{n}\right)=o\left(\frac{1}{k}\right) \quad \text { as } n \rightarrow \infty .
$$

Finally, using (6.6) and (6.8) in (6.3), we see that we also have

$$
\liminf _{n \rightarrow \infty} \mathbb{P}\left(\mathscr{M}_{n}^{U} \leq u_{n}\right) \geq \exp \left\{-K_{A} \mathbb{E}_{\pi_{\mathbb{D}}}[\tau] u^{-\alpha}\right\} .
$$


LEMMA 6.3. Suppose that $\left(\mathrm{H}_{1}\right),\left(\mathrm{H}_{2}\right)$ and $\left(\mathrm{H}_{3}\right)$ are satisfied and $d_{A}$ is bounded and continuous. Then for any $\Delta>0$, there exists a constant $\delta>0$ such that

$$
\limsup _{n \rightarrow \infty} \mathbb{P}\left(\max _{|m-n|<n \delta}\left|\mathscr{M}_{m}-\mathscr{M}_{n}\right|>n^{1 / \alpha} \Delta\right) \leq \Delta
$$

Proof. Let $k \in \mathbb{N}_{+}$. Then $\mathscr{M}_{n+k}=\max \left\{\mathscr{M}_{n}, V_{n+1}^{A}, \ldots, V_{n+k}^{A}\right\}$, and hence

$$
\mathscr{M}_{n} \leq \mathscr{M}_{n+k} \leq \mathscr{M}_{n}+\max \left\{V_{n+1}^{A}, \ldots, V_{n+k}^{A}\right\} .
$$

Since $V_{n}^{A}:=V_{n} / d_{A}\left(\tilde{V}_{n}\right)$, it follows that

$$
\max _{|m-n|<n \delta}\left|\mathscr{M}_{m}-\mathscr{M}_{n}\right| \leq b \max \left\{\left|V_{\lfloor n-n \delta\rfloor+1}\right|, \ldots,\left|V_{\lfloor n+n \delta\rfloor}\right|\right\},
$$

where $b=\max \left\{\left(d_{A}(x)\right)^{-1}: x \in \mathbb{S}_{+}^{d-1}\right\}<\infty$. We now determine the maximum on the right-hand side, conditioned on $\left\{V_{\lfloor n-n \delta\rfloor}=v\right\}$. Set

$$
m_{n}=\lfloor n+n \delta\rfloor-(\lfloor n-n \delta\rfloor+1) \leq 2 n \delta,
$$

and observe that as an upper bound, it is sufficient to study $\mathscr{M}_{m_{n}}$ conditioned on $\left\{V_{0}=v\right\}$.

Let $\mathbb{D} \subset \mathbb{R}_{+}^{d}$ be chosen such that $\pi\left(\mathbb{D}^{c}\right) \leq \Delta / 2$, and let $v \in \mathbb{D} \backslash\{0\}$. Since $\mathscr{M}_{n} \leq$ $\mathscr{M}_{n}^{U}$ and $m_{n} \leq 2 n \delta$, we obtain from Proposition 6.2 (with $A=\left\{x \in \mathbb{R}_{+}^{d}:|x|>1\right\}$ ) that

$$
\begin{aligned}
& \limsup _{n \rightarrow \infty} \mathbb{P}\left(\mathscr{M}_{m_{n}}>n^{1 / \alpha} \Delta \mid V_{0}=v\right) \\
& \quad \leq 1-\exp \left\{-K_{A} \mathbb{E}_{\pi_{\mathbb{D}}}[\tau] \cdot 2 \delta \Delta^{-\alpha}\right\} \\
& \quad=2 K_{A} \mathbb{E}_{\pi_{\mathbb{D}}}[\tau] \Delta^{-\alpha} t \quad \text { where } t \in(0, \delta),
\end{aligned}
$$

and the right-hand side is $\leq \Delta / 2$ when $\delta$ is chosen sufficiently small. Note that (6.11) holds for all $v \in \mathbb{D} \backslash\{0\}$. Finally, let $\gamma_{n}$ denote the distribution function of $V_{\lfloor n-n \delta\rfloor}$. By the positive Harris recurrence of $\left\{V_{n}\right\}$ (Lemma 3.4), we have that $\left|\gamma_{n}-\pi\right|_{\mathrm{TV}} \rightarrow 0$ as $n \rightarrow \infty$. Then, using Fatou's lemma, we deduce that

$$
\begin{aligned}
& \limsup _{n \rightarrow \infty} \mathbb{P}\left(\max _{|m-n|<n \delta}\left|\mathscr{M}_{m}-\mathscr{M}_{n}\right|>n^{1 / \alpha} \Delta\right) \\
& \quad \leq \limsup _{n \rightarrow \infty}\left(\gamma_{n}\left(\mathbb{D}^{c}\right)+\left|\gamma_{n}-\pi\right|_{\mathrm{TV}}+\int_{\mathbb{D}} \mathbb{P}\left(\mathscr{M}_{m_{n}}>n^{1 / \alpha} \Delta \mid V_{0}=v\right) \pi(d v)\right) \\
& \quad \leq \pi\left(\mathbb{D}^{c}\right)+\frac{\Delta}{2} \pi(\mathbb{D}) \leq \Delta .
\end{aligned}
$$

Proof of TheOrem 2.8. Assuming that $d_{A}$ is bounded, the first assertion follows from Corollary 4.2, equation (4.2), and the uniformity provided by 
Lemma 4.6. To remove the assumption that $d_{A}$ is bounded, see Step 4 below. To establish the remaining assertion, we proceed in four steps.

STEP 1. First, assume that $d_{A}$ is bounded and continuous and that $\left(\mathrm{H}_{3}\right)$ is satisfied. We claim that

$$
\lim _{n \rightarrow \infty} \mathbb{P}\left(\mathscr{M}_{n} \leq n^{1 / \alpha} u\right)=e^{-K_{A} u^{-\alpha}} ;
$$

that is to say, we can transfer the result for maxima over cycles (Proposition 6.2) to the process of running maxima, namely to $\mathscr{M}_{n}$.

To establish an upper bound for $\limsup _{n \rightarrow \infty} \mathbb{P}\left(\mathscr{M}_{n} \leq n^{1 / \alpha} u\right)$, observe that, by definition, $\mathscr{M}_{N_{\mathbb{D}}(n)}^{U}$ corresponds to the value of $\left\{\mathscr{M}_{j}\right\}$ during its last visit to $\mathbb{D}$ in the time interval $[0, n]$. Thus,

$$
\mathbb{P}\left(\mathscr{M}_{n}>n^{1 / \alpha} u\right) \geq \mathbb{P}\left(\mathscr{M}_{N_{\mathbb{D}}(n)}^{U}>n^{1 / \alpha} u\right) .
$$

To replace the random time $N_{\mathbb{D}}(n)$ by a fixed time, observe by Lemma 3.3 that for all $\delta>0$,

$$
\mathbb{P}\left(\left|\frac{N_{\mathbb{D}}(n)}{n}-\pi(\mathbb{D})\right| \geq \delta\right) \rightarrow 0 \quad \text { as } n \rightarrow \infty .
$$

Set $t_{n}=n(\pi(\mathbb{D})-\delta)$ and $\Omega_{n}=\left\{\left|\left(N_{\mathbb{D}}(n) / n\right)-\pi(\mathbb{D})\right|<\delta\right\}$, and note that $N_{\mathbb{D}}(n) \geq$ $\left\lfloor t_{n}\right\rfloor$ on $\Omega_{n}$. Then

$$
\mathbb{P}\left(\mathscr{M}_{N_{\mathbb{D}}(n)}^{U}>n^{1 / \alpha} u\right) \geq \mathbb{P}\left(\mathscr{M}_{\left\lfloor t_{n}\right\rfloor}^{U}>n^{1 / \alpha} u\right)-\mathbb{P}\left(\Omega_{n}^{c}\right) .
$$

Then combining (6.13), (6.14) and (6.15) and applying Proposition 6.2, we conclude that for all $\delta>0$,

$$
\begin{aligned}
\liminf _{n \rightarrow \infty} \mathbb{P}\left(\mathscr{M}_{n}>n^{1 / \alpha} u\right) & \geq \lim _{n \rightarrow \infty} \mathbb{P}\left(\mathscr{M}_{\left\lfloor t_{n}\right\rfloor}^{U}>n^{1 / \alpha} u\right) \\
& =1-\exp \left\{-K_{A} \mathbb{E}_{\pi_{\mathbb{D}}}[\tau](\pi(\mathbb{D})-\delta) u^{-\alpha}\right\} .
\end{aligned}
$$

Hence, letting $\delta \downarrow 0$ and recalling that $\pi(\mathbb{D})=\left(\mathbb{E}_{\pi_{\mathbb{D}}}[\tau]\right)^{-1}$ (Lemma 3.3), we obtain that

$$
\limsup _{n \rightarrow \infty} \mathbb{P}\left(\mathscr{M}_{n} \leq n^{1 / \alpha} u\right) \leq \exp \left\{-K_{A} u^{-\alpha}\right\}
$$

To establish the corresponding lower bound for $\mathbb{P}\left(\mathscr{M}_{n} \leq n^{1 / \alpha} u\right)$, observe that for any $\Delta>0$,

$$
\begin{aligned}
\mathbb{P}\left(\mathscr{M}_{n}>n^{1 / \alpha} u\right) \leq & \mathbb{P}\left(\mathscr{M}_{N_{\mathbb{D}}(n)}^{U}>n^{1 / \alpha}(u-\Delta)\right) \\
& +\mathbb{P}\left(\left|\mathscr{M}_{n}-\mathscr{M}_{N_{\mathbb{D}}(n)}^{U}\right|>n^{1 / \alpha} \Delta\right) .
\end{aligned}
$$

Reasoning as before, we see that the first term on the right-hand side satisfies

$$
\limsup _{n \rightarrow \infty} \mathbb{P}\left(\mathscr{M}_{N_{\mathbb{D}}(n)}^{U}>n^{1 / \alpha}(u-\Delta)\right) \leq 1-\exp \left\{-K_{A}(u-\Delta)^{-\alpha}\right\} .
$$


Now to quantify the second term on the right-hand side of (6.17), recall that $\mathscr{M}_{N_{\mathbb{D}}(n)}^{U}$ is the value of the process $\left\{\mathscr{M}_{j}\right\}$ during its last visit to $\mathbb{D}$ in the interval $[0, n]$. Since $\kappa_{i}$ denotes the time of the $i$ th visit to $\mathbb{D}$, it follows by definition that $\mathscr{M}_{N_{\mathbb{D}}(n)}^{U}=\mathscr{M}_{\kappa_{N_{\mathbb{D}}(n)}}$. Moreover, for any $\delta>0$, we obtain by Lemma 3.3 that

$$
\mathbb{P}\left(\left|\frac{\kappa_{N_{\mathbb{D}}(n)}}{n}-1\right| \geq \delta\right) \rightarrow 0 \quad \text { as } n \rightarrow \infty .
$$

Set $\widehat{\Omega}_{n}=\left\{\left|\left(\kappa_{N_{\mathbb{D}}(n)} / n\right)-1\right|<\delta\right\}$. Then

$$
\begin{aligned}
\mathbb{P}\left(\left|\mathscr{M}_{n}-\mathscr{M}_{N_{\mathbb{D}}(n)}^{U}\right|>n^{1 / \alpha} \Delta\right) & \leq \mathbb{P}\left(\left|\mathscr{M}_{n}-\mathscr{M}_{N_{\mathbb{D}}(n)}^{U}\right|>n^{1 / \alpha} \Delta ; \widehat{\Omega}_{n}\right)+\mathbb{P}\left(\widehat{\Omega}_{n}^{c}\right) \\
& \leq \mathbb{P}\left(\max _{|m-n|<n \delta}\left|\mathscr{M}_{m}-\mathscr{M}_{n}\right|>n^{1 / \alpha} \Delta\right)+o(1)
\end{aligned}
$$

as $n \rightarrow \infty$. Hence, by Lemma 6.3,

$$
\limsup _{n \rightarrow \infty} \mathbb{P}\left(\left|\mathscr{M}_{n}-\mathscr{M}_{N_{\mathbb{D}}(n)}^{U}\right|>n^{1 / \alpha} \Delta\right) \leq \Delta .
$$

Finally, substituting (6.18) and (6.19) into (6.17) and letting $\Delta \rightarrow 0$, we conclude that $\liminf _{n \rightarrow \infty} \mathbb{P}\left(\mathscr{M}_{n} \leq n^{1 / \alpha} u\right) \geq \exp \left\{-K_{A} u^{-\alpha}\right\}$. Together with (6.16), the assertion follows.

STEP 2. Next, we remove the additional assumption $\left(\mathrm{H}_{3}\right)$, but still assume that the function $d_{A}$ is bounded and continuous.

To remove $\left(\mathrm{H}_{3}\right)$, we employ the smoothing argument introduced in Section 5. Let $\left\{\left(\widehat{M}_{n}, \widehat{Q}_{n}\right): n=0,1, \ldots\right\}$ be defined as in (5.8). Then, since we are assuming here that $k=1$ in $(\mathfrak{K})$, it follows that $\left(\widehat{M}_{n}, \widehat{Q}_{n}\right)=\left(M_{n}, Q_{n}\right)$ for all $n \in \mathbb{N}_{+}$. This yields $V_{n, \varepsilon} \leq V_{n} \leq V_{n}^{\varepsilon}$ for all $n \in \mathbb{N}_{+}$.

By repeating the computation leading to (5.14), we obtain

$$
\left|\widetilde{V}_{n, \varepsilon}-\widetilde{V}_{n}\right| \leq \frac{2 \varepsilon}{s} \quad \text { and } \quad\left|\widetilde{V}_{n}^{\varepsilon}-\widetilde{V}_{n}\right| \leq \frac{2 \varepsilon}{s} \quad \text { for all } n \in \mathbb{N}_{+} .
$$

Since $d_{A}$ is assumed to be continuous on the compact set $\mathbb{S}_{+}^{d-1}$, it is, in fact, equicontinuous. Hence, there is a sequence $\delta(\varepsilon)$, tending to zero as $\varepsilon \rightarrow 0$, such that

$\left|d_{A}\left(\widetilde{V}_{n, \varepsilon}\right)-d_{A}\left(\widetilde{V}_{n}\right)\right| \leq \delta(\varepsilon) \quad$ and $\quad\left|d_{A}\left(\widetilde{V}_{n}^{\varepsilon}\right)-d_{A}\left(\widetilde{V}_{n}\right)\right| \leq \delta(\varepsilon) \quad$ for all $n \in \mathbb{N}_{+}$.

Since $A \subset\{v:|v|>1\} \Rightarrow d_{A}>1$, we have

$$
\begin{aligned}
& \left|V_{n}\right| \leq u d_{A}\left(\widetilde{V}_{n}\right) \\
& \quad \Rightarrow \quad\left|V_{n, \varepsilon}\right| \leq u d_{A}\left(\widetilde{V}_{n}\right) \leq u d_{A}\left(\widetilde{V}_{n, \varepsilon}\right)+u \delta(\varepsilon) \leq u d_{A}\left(\widetilde{V}_{n, \varepsilon}\right)(1+\delta(\varepsilon)) .
\end{aligned}
$$

Thus, $\mathbb{P}\left(\mathscr{M}_{n} \leq u\right) \leq \mathbb{P}\left(\mathscr{M}_{n, \varepsilon} \leq u(1+\delta(\varepsilon))\right)$. Similarly, for all $n \in \mathbb{N}_{+}$,

$$
\left|V_{n}^{\varepsilon}\right| \leq u d_{A}\left(\widetilde{V}_{n}^{\varepsilon}\right)(1-\delta(\varepsilon)) \quad \Rightarrow \quad\left|V_{n}\right| \leq u d_{A}\left(\widetilde{V}_{n}^{\varepsilon}\right)-u \delta(\varepsilon) \leq u d_{A}\left(\widetilde{V}_{n}\right),
$$


and we obtain that $\mathbb{P}\left(\mathscr{M}_{n} \leq u\right) \geq \mathbb{P}\left(\mathscr{M}_{n}^{\varepsilon} \leq u(1-\delta(\varepsilon))\right)$. Using these upper and lower bounds together with Step 1 , we conclude that

$$
\begin{aligned}
\exp \{ & \left.-K^{\varepsilon}(u-\delta(\varepsilon))^{-\alpha}\right\} \\
& \leq \liminf _{n \rightarrow \infty} \mathbb{P}\left(\mathscr{M}_{n} \leq n^{1 / \alpha} u\right) \\
& \leq \limsup _{n \rightarrow \infty} \mathbb{P}\left(\mathscr{M}_{n} \leq n^{1 / \alpha} u\right) \leq \exp \left\{-K_{\varepsilon}(u+\delta(\varepsilon))^{-\alpha}\right\},
\end{aligned}
$$

for constants $K_{\varepsilon}:=C_{\varepsilon} D_{A}$ and $K^{\varepsilon}:=C^{\varepsilon} D_{A}$, where $C_{\varepsilon}$ and $C^{\varepsilon}$ are given as in Section 5. By Lemma 5.6, this yields (6.12).

STEP 3. We now relate the behavior of the maxima to the behavior of the first passage times. Recall that $V_{n} \in u A \Leftrightarrow\left|V_{n}\right|>u d_{A}\left(\widetilde{V}_{n}\right) \Leftrightarrow\left|V_{n}^{A}\right|>u$. Hence, for all $n \in \mathbb{N}_{+}$and all $u>0$,

$$
\mathbb{P}\left(T_{u}^{A} \leq n\right)=\mathbb{P}\left(\left|V_{i}^{A}\right|>u, \text { some } 1 \leq i \leq n\right)=\mathbb{P}\left(\mathscr{M}_{n}>u\right) .
$$

Then by (6.12) and (6.21), $\lim _{n \rightarrow \infty} \mathbb{P}\left(T_{n^{1 / \alpha}}^{A} w \leq n\right)=1-e^{-K_{A} w^{-\alpha}}$; and setting $u=n^{1 / \alpha} w$ and $z=w^{-\alpha}$ yields

$$
\lim _{u \rightarrow \infty} \mathbb{P}\left(\frac{T_{u}^{A}}{u^{\alpha}} \leq z\right)=1-e^{-K_{A} z}, \quad z \geq 0 .
$$

STEP 4. Finally, suppose that $\mathfrak{P}_{A}:=\left\{x \in \mathbb{S}_{+}^{d-1}: d_{A}(x)<\infty\right\} \neq \mathbb{S}_{+}^{d-1}$. For any $L \geq 1$, set

$$
\mathscr{K}_{L}=\left\{w \in \mathbb{R}_{+}^{d}:|w| \geq L\right\} \quad \text { and } \quad A_{L}=A \cup \mathscr{K}_{L} .
$$

First, observe that $d_{\mathscr{K}_{L}}(x):=\inf \left\{t: t x \in \mathscr{K}_{L}\right\}=L, \forall x \in \mathbb{S}_{+}^{d-1}$. Hence, letting $r_{\alpha}^{\mathscr{K}_{L}}$ be defined as in (2.18), we have that $r_{\alpha}^{\mathscr{K}_{L}}(x)=L^{\alpha} r_{\alpha}(x) \uparrow \infty$ as $L \rightarrow \infty$ (uniformly in $x$, by Lemma 2.2). Now in general, the constant $K_{A}$ is proportional to $D_{A}$, where the latter constant was characterized in Lemma 6.1. Using this characterization, we see that $r_{\alpha}^{\mathscr{K}_{L}}(x) \uparrow \infty, \forall x \Rightarrow D_{A}^{\mathscr{K}_{L}} \downarrow 0$ as $L \rightarrow \infty$. Consequently,

$$
\Delta(L):=\lim _{u \rightarrow \infty} \mathbb{P}\left(\frac{T_{u}^{\mathscr{K}_{L}}}{u^{\alpha}} \leq z\right) \searrow 0 \quad \text { as } L \rightarrow \infty .
$$

Since

$$
\left|\mathbb{P}\left(\frac{T_{u}^{A_{L}}}{u^{\alpha}} \leq z\right)-\mathbb{P}\left(\frac{T_{u}^{A}}{u^{\alpha}} \leq z\right)\right| \leq \mathbb{P}\left(\frac{T_{u}^{\mathscr{K}_{L}}}{u^{\alpha}} \leq z\right),
$$

we conclude that for all $z \geq 0$,

$$
\begin{aligned}
\lim _{u \rightarrow \infty} \mathbb{P}\left(\frac{T_{u}^{A_{L}}}{u^{\alpha}} \leq z\right)-\Delta(L) & \leq \liminf _{u \rightarrow \infty} \mathbb{P}\left(\frac{T_{u}^{A}}{u^{\alpha}} \leq z\right) \leq \limsup _{u \rightarrow \infty} \mathbb{P}\left(\frac{T_{u}^{A}}{u^{\alpha}} \leq z\right) \\
& \leq \lim _{u \rightarrow \infty} \mathbb{P}\left(\frac{T_{u}^{A_{L}}}{u^{\alpha}} \leq z\right)+\Delta(L) .
\end{aligned}
$$


Thus, by (6.23) and Step 3,

$$
\lim _{u \rightarrow \infty} \mathbb{P}\left(\frac{T_{u}^{A}}{u^{\alpha}} \leq z\right)=1-\lim _{L \rightarrow \infty} \exp \left\{-\left(C D_{A_{L}}\right) z\right\}:=1-\exp \left\{-\left(C D_{A}\right) z\right\} .
$$

Observe that $D_{A}:=\lim _{L \rightarrow \infty} D_{A_{L}}$ exists, since $D_{A_{L}}=u^{\alpha} \mathbb{P}\left(\mathfrak{T}_{u}^{A_{L}}<\infty \mid V_{0} \sim \pi_{\mathbb{D}}\right)$ is a decreasing sequence; namely, it represents the hitting probability of a decreasing sequence of sets.

It remains to identify $D_{A}$ as the ruin constant in this case. Arguing as before, we have that for all $u>0$,

$$
\left|u^{\alpha} \mathbb{P}\left(\mathfrak{T}_{u}^{A_{L}}<\infty \mid V_{0}=v\right)-u^{\alpha} \mathbb{P}\left(\mathfrak{T}_{u}^{A}<\infty \mid V_{0}=v\right)\right| \leq u^{\alpha} \mathbb{P}\left(\mathfrak{T}_{u}^{\mathscr{K}_{L}}<\infty \mid V_{0}=v\right),
$$

which tends to zero as $L \rightarrow \infty$. Thus, by another sandwich argument,

$$
\begin{aligned}
\lim _{u \rightarrow \infty} u^{\alpha} \mathbb{P}\left(\mathfrak{T}_{u}^{A}<\infty \mid V_{0}=v\right) & =\lim _{L \rightarrow \infty}\left(\lim _{u \rightarrow \infty} u^{\alpha} \mathbb{P}\left(\mathfrak{T}_{u}^{A_{L}}<\infty \mid V_{0}=v\right)\right) \\
& =r_{\alpha}(\widetilde{v}) \lim _{L \rightarrow \infty} D_{A_{L}}=r_{\alpha}(\widetilde{v}) D_{A},
\end{aligned}
$$

which gives the required identification of $D_{A}$ as the constant in the ruin problem for the Markov random walk; cf. Lemma 6.1.

To conclude the proof, observe that the same reasoning yields (2.22) for unbounded functions $d_{A}$; namely, one can again introduce the family $A_{L}=A \cup \mathscr{K}_{L}$ for $L \geq 1$, and argue that the hitting probability of the set $\mathscr{K}_{L}$-now prior to the return time $\tau$-becomes asymptotically negligible as $L \rightarrow \infty$. The argument is entirely identical, so we omit the details.

7. Determining the path of large exceedance. We conclude by providing the proofs of Theorems 2.11 and 2.12. (For a stronger version of Theorem 2.11, allowing paths of infinite length, see the arXiv version [16].)

Proof OF THEOREM 2.11. It follows by Theorem 2.8 that

$$
\lim _{u \rightarrow \infty} u^{\alpha} \mathbb{E}_{v}\left[\mathbf{1}_{\left\{T_{u}^{A}<\tau\right\}}\right]=C(v) \int \frac{e^{-\alpha s}}{r_{\alpha}^{A}(x)} \varrho^{A}(d x, d s)=C(v) D_{A},
$$

where $C(v)=r_{\alpha}(\widetilde{v}) \mathbb{E}_{\boldsymbol{\delta}_{v}}^{\alpha}\left[|Z|^{\alpha} \mathbf{1}_{\{\tau=\infty\}}\right]$ and $D_{A}$ is given as in (2.20). Thus, to establish the result, it suffices to show that

$$
\begin{aligned}
\lim _{u \rightarrow \infty} u^{\alpha} \mathbb{E}_{v}\left[g\left(\frac{V_{I_{u}}}{\left|V_{I_{u}}\right|}, \ldots, \frac{V_{I_{u}+m}}{\left|V_{I_{u}}\right|}\right) \mathbf{1}_{\left\{T_{u}^{A}<\tau\right\}}\right] \\
=C(v) D_{A} \int \mathbb{E}_{x}^{\alpha}\left[g\left(X_{0}, e^{S_{1}} X_{1}, \ldots, e^{S_{m}} X_{m}\right)\right] \varrho(d x, d s) .
\end{aligned}
$$


To verify (7.1), proceed as in the proof of Proposition 4.1, first converting to the $\alpha$-shifted measure to obtain that

$$
\begin{aligned}
u^{\alpha} \mathbb{E}_{v} & {\left[g\left(\frac{V_{I_{u}}}{\left|V_{I_{u}}\right|}, \ldots, \frac{V_{I_{u}+m}}{\left|V_{I_{u}}\right|}\right) \mathbf{1}_{\left\{T_{u}^{A}<\tau\right\}}\right] } \\
& =u^{\alpha} r_{\alpha}(\widetilde{v}) \mathbb{E}_{\boldsymbol{\delta}_{v}}^{\alpha}\left[\frac{e^{-\alpha S_{T_{u}^{A}}}}{r_{\alpha}\left(X_{\left.T_{u}^{A}\right)}\right.} g\left(\frac{V_{I_{u}}}{\left|V_{I_{u}}\right|}, \ldots, \frac{V_{I_{u}+m}}{\left|V_{I_{u}}\right|}\right) \mathbf{1}_{\left\{T_{u}^{A}<\tau\right\}}\right] \\
& =r_{\alpha}(\widetilde{v}) \mathbb{E}_{\boldsymbol{\delta}_{v}}^{\alpha}\left[\left|Z_{T_{u}^{A}}\right|^{\alpha} \mathfrak{G}_{u} \mathbf{1}_{\left\{T_{u}^{A}<\tau\right\}}\right],
\end{aligned}
$$

where $Z_{n}:=\left|V_{n}\right| / e^{S_{n}}, n=0,1, \ldots$, and

$$
\mathfrak{G}_{u}:=\frac{1}{r_{\alpha}^{A}\left(X_{T_{u}^{A}}\right)}\left(\frac{\left|V_{T_{u}^{A}}^{A}\right|}{u}\right)^{-\alpha}\left(\frac{d_{A}\left(X_{T_{u}^{A}}\right)}{d_{A}\left(\widetilde{V}_{T_{u}^{A}}\right)}\right)^{\alpha} g\left(\frac{V_{I_{u}}}{\left|V_{I_{u}}\right|}, \ldots, \frac{V_{I_{u}+m}}{\left|V_{I_{u}}\right|}\right) .
$$

[The term $\left(d_{A}\left(X_{T_{u}^{A}}\right) / d_{A}\left(\widetilde{V}_{T_{u}^{A}}\right)\right)^{\alpha}$ arises when replacing $\left|V_{T_{u}^{A}}\right|^{-\alpha} / r_{\alpha}\left(X_{T_{u}^{A}}\right)$ with $\left|V_{T_{u}^{A}}^{A}\right|^{-\alpha} / r_{\alpha}^{A}\left(X_{T_{u}^{A}}\right)$, as can be seen directly from the definitions (2.18) and (3.32).] The right-hand side of (7.2) can be written as the difference of two terms, namely

$$
\begin{gathered}
r_{\alpha}(\widetilde{v}) \mathbb{E}_{\delta_{v}}^{\alpha}\left[\left(\left|Z_{T_{u}^{A}}\right|^{\alpha} \mathbf{1}_{\left\{T_{u}^{A}<\tau\right\}}-\left|Z_{n}\right|^{\alpha} \mathbf{1}_{\left\{n \leq T_{u}^{A}\right\}} \mathbf{1}_{\{n \leq \tau\}}\right) \mathfrak{G}_{u}\right] \\
+r_{\alpha}(\widetilde{v}) \mathbb{E}_{\boldsymbol{\delta}_{v}}^{\alpha}\left[\left|Z_{n}\right|^{\alpha} \mathbf{1}_{\left\{n \leq T_{u}^{A}\right\}} \mathbf{1}_{\{n \leq \tau\}} \mathbb{E}^{\alpha}\left[\mathfrak{G}_{u} \mid \mathscr{F}_{n}\right]\right] .
\end{gathered}
$$

As in the proof of Proposition 4.1, we may then apply Lemma 4.3(i) and use the uniform boundedness of $\left\{\mathfrak{G}_{u}\right\}$ to conclude that the first term in (7.4) tends to zero as $u \rightarrow \infty$ and then $n \rightarrow \infty$.

Thus, it suffices to analyze the second term in (7.4). Reasoning again as in the proof of Proposition 4.1, it suffices to show that

$$
\lim _{u \rightarrow \infty} \mathbb{E}^{\alpha}\left[\mathfrak{G}_{u} \mid \mathscr{F}_{n}\right]=D_{A} \lim _{u \rightarrow \infty} \mathbb{E}_{X_{n}, V_{n}}^{\alpha}\left[g\left(\frac{V_{I_{u}}}{\left|V_{I_{u}}\right|}, \ldots, \frac{V_{I_{u}+m}}{\left|V_{I_{u}}\right|}\right)\right] \quad \mathbb{P}^{\alpha} \text {-a.s. }
$$

Now to establish (7.5), introduce a further conditioning on $\mathscr{F}_{I_{u}+m}$ inside $\mathbb{E}^{\alpha}\left[\mathfrak{G}_{u} \mid\right.$ $\left.\mathscr{F}_{n}\right]$. Then by the nonlinear renewal theorem (Theorem 3.8) together with Lemma 3.10,

$$
D(u):=\mathbb{E}_{X_{I_{u}+m}, V_{I_{u}+m}}^{\alpha}\left[\frac{1}{r_{\alpha}^{A}\left(\widetilde{V}_{T_{u}^{A}}\right)}\left(\frac{\left|V_{T_{u}^{A}}^{A}\right|}{u}\right)^{-\alpha}\left(\frac{d_{A}\left(X_{T_{u}^{A}}\right)}{d_{A}\left(\widetilde{V}_{T_{u}^{A}}\right)}\right)^{\alpha} \frac{r_{\alpha}^{A}\left(\widetilde{V}_{T_{u}^{A}}\right)}{r_{\alpha}^{A}\left(X_{T_{u}^{A}}\right)}\right] \mathbf{1}_{\left\{I_{u}+m<T_{u}^{A}\right\}}
$$

converges to $D_{A}$ in $\mathbb{P}^{\alpha}$-probability. Then on $\left\{n \leq T_{u}^{A}\right\}$,

$$
\begin{aligned}
\lim _{u \rightarrow \infty} \mathbb{E}^{\alpha}\left[\mathfrak{G}_{u} \mid \mathscr{F}_{n}\right]= & \lim _{u \rightarrow \infty} \mathbb{E}_{X_{n}, V_{n}}^{\alpha}\left[\left(D(u)-D_{A}\right) g\left(\frac{V_{I_{u}}}{\left|V_{I_{u}}\right|}, \ldots, \frac{V_{I_{u}+m}}{\left|V_{I_{u}}\right|}\right)\right] \\
& +\lim _{u \rightarrow \infty} D_{A} \mathbb{E}_{X_{n}, V_{n}}^{\alpha}\left[g\left(\frac{V_{I_{u}}}{\left|V_{I_{u}}\right|}, \ldots, \frac{V_{I_{u}+m}}{\left|V_{I_{u}}\right|}\right)\right] \quad \mathbb{P}^{\alpha} \text {-a.s., }
\end{aligned}
$$


and the first term on the right-hand side vanishes by dominated convergence. This completes the proof of (7.5), and hence the theorem.

Proof of THEOREM 2.12. It suffices to show that

$$
\limsup _{u \rightarrow \infty} u^{\alpha} \mathbb{E}_{v}\left[\left|\frac{1}{T_{u}^{A}} \sum_{n=1}^{T_{u}^{A}} g\left(\log \left(\frac{\left|V_{n}\right|}{\left|V_{n-1}\right|}\right)\right)-\widehat{\mathbb{E}}^{\alpha}\left[g\left(S_{1}\right)\right]\right| \mathbf{1}_{\left\{T_{u}^{A}<\tau\right\}}\right]=0 .
$$

For simplicity, introduce the shorthand notation $\mu_{g}:=\widehat{\mathbb{E}}^{\alpha}\left[g\left(S_{1}\right)\right]$ and $\Sigma_{i}^{n}:=$ $\sum_{j=i}^{n} g\left(\log \left|V_{j}\right|-\log \left|V_{j-1}\right|\right)$.

Let $\left\{\varepsilon_{u}\right\}_{u>0}$ be a sequence such that $\varepsilon_{u}=o(u)$ and $\varepsilon_{u} \uparrow \infty$ as $u \rightarrow \infty$. Let $\gamma_{u}=u-\varepsilon_{u}$ and $J_{u}=T_{\gamma_{u}}^{A}$, and set $B_{1}=\max _{x, y}\left(r_{\alpha}(x) / r_{\alpha}(y)\right)$. Then from a change of measure argument, we infer that

$$
\begin{aligned}
& u^{\alpha} \mathbb{E}\left[\left|\frac{1}{T_{u}^{A}} \Sigma_{1}^{T_{u}^{A}}-\mu_{g}\right| \mathbf{1}_{\left\{T_{u}^{A}<\tau\right\}} \mid V_{0}=v\right] \\
& \quad=r_{\alpha}(\widetilde{v}) \mathbb{E}_{\delta_{v}}^{\alpha}\left[\frac{e^{-\alpha\left(S_{T_{u}^{A}}-\log u\right)}}{r_{\alpha}\left(X_{T_{u}^{A}}\right)}\left|\frac{1}{T_{u}^{A}} \Sigma_{1}^{T_{u}^{A}}-\mu_{g}\right| \mathbf{1}_{\left\{T_{u}^{A}<\tau\right\}}\right] \\
& \quad \leq B_{1} \mathbb{E}_{\delta_{v}}^{\alpha}\left[\left|\frac{1}{J_{u}} \Sigma_{1}^{J_{u}}-\mu_{g}\right|\right]+B_{1} \mathbb{E}_{\delta_{v}}^{\alpha}\left[\left|\frac{1}{J_{u}} \Sigma_{1}^{J_{u}}-\frac{1}{T_{u}^{A}} \Sigma_{1}^{T_{u}^{A}}\right|\right] \\
& :=\mathbb{I}_{1}(u)+\mathbb{I}_{2}(u) .
\end{aligned}
$$

First, consider $\mathbb{I}_{2}(u)$. Note $\left|J_{u}^{-1} \Sigma_{1}^{J_{u}}-\left(T_{u}^{A}\right)^{-1} \Sigma_{1}^{T_{u}^{A}}\right|$ is bounded above by

$$
\left|\frac{1}{J_{u}}\left(\Sigma_{1}^{J_{u}}-\Sigma_{1}^{T_{u}^{A}}\right)\right|+\left|\left(\frac{1}{J_{u}}-\frac{1}{T_{u}^{A}}\right) \Sigma_{1}^{T_{u}^{A}}\right| \leq 2\left|\frac{T_{u}^{A}-J_{u}}{J_{u}}\right| \cdot|g|_{\infty} .
$$

By Lemma 3.12, $\left(J_{u} / \log u\right) \rightarrow\left(\lambda^{\prime}(\alpha)\right)^{-1}$ and $\left(\left(T_{u}^{A}-J_{u}\right) / \log u\right) \rightarrow 0$ in $\mathbb{P}^{\alpha}{ }_{-}$ probability. Hence, the term inside the expectation in $\mathbb{I}_{2}(u)$ tends to zero in $\mathbb{P}^{\alpha}$ probability, and furthermore is bounded above by $2|g|_{\infty}$. Thus,

$$
\limsup _{u \rightarrow \infty} \mathbb{I}_{2}(u)=0 \text {. }
$$

To study $\mathbb{I}_{1}(u)$, observe that $\left|J_{u}^{-1} \Sigma_{1}^{J_{u}}-\mu_{g}\right|$ is bounded above by

$$
\frac{1}{J_{u}} \sum_{n=1}^{J_{u}}\left|g\left(\log \left(\frac{\left|V_{n}\right|}{\left|V_{n-1}\right|}\right)\right)-g\left(S_{n}-S_{n-1}\right)\right|+\left|\frac{1}{J_{u}} \sum_{n=1}^{J_{u}} g\left(S_{n}-S_{n-1}\right)-\mu_{g}\right| \text {. }
$$

By Lemma 3.12, $J_{u} \uparrow \infty$ a.s. as $u \rightarrow \infty$. The second term tends to zero $\mathbb{P}^{\alpha}$-a.s., by [10], Lemma 6.1. Next, use the Lipschitz continuity of $g$ to infer that for some finite constant $B_{g}$, the first term is bounded above by

$$
\frac{B_{g}}{J_{u}} \sum_{n=1}^{J_{u}}\left(|\log | V_{n}|-\log | V_{n-1}\left|-\left(S_{n}-S_{n-1}\right)\right|\right) .
$$


Also, it follows directly from the definitions [as given in (2.5) and (3.9)] that

$$
|\log | V_{n}|-\log | V_{n-1}\left|-\left(S_{n}-S_{n-1}\right)\right|:=|\log | Z_{n}|-\log | Z_{n-1}|| .
$$

Now by Lemma 3.6, $\left\{\left|Z_{n}\right|\right\}$ converges a.s. to the proper random variable $|Z|$ (and thus forms a Cauchy sequence). Then by Césaro's theorem,

$$
\limsup _{u \rightarrow \infty} \frac{1}{J_{u}} \sum_{n=1}^{J_{u}}|\log | Z_{n}|-\log | Z_{n-1}||=0 \quad \text { a.s., }
$$

and we conclude by an application of Fatou's lemma that $\lim \sup _{u \rightarrow \infty} \mathbb{I}_{1}(u)=0$ $\mathbb{P}^{\alpha}$-a.s. This establishes (2.28).

Acknowledgments. Part of the research was done while S.M. held positions at WWU Münster and the University of Wroclaw, for which he expresses gratitude.

\section{REFERENCES}

[1] AlsmeYeR, G. (1991). Erneuerungstheorie: Analyse stochastischer Regenerationsschemata. B. G. Teubner, Stuttgart. MR1119301

[2] Alsmeyer, G. (1997). The Markov renewal theorem and related results. Markov Process. Related Fields 3 103-127.

[3] Alsmeyer, G. (2003). On the Harris recurrence of iterated random Lipschitz functions and related convergence rate results. J. Theoret. Probab. 16 217-247. MR1956829

[4] Alsmeyer, G. (2016). On the stationary tail index of iterated random Lipschitz functions. Stochastic Process. Appl. 126 209-233. MR3426517

[5] Asmussen, S. (1982). Conditioned limit theorems relating a random walk to its associate, with applications to risk reserve processes and the $G I / G / 1$ queue. Adv. in Appl. Probab. 14 143-170. MR0644012

[6] BAsraK, B., Davis, R. A. and Mikosch, T. (2002). A characterization of multivariate regular variation. Ann. Appl. Probab. 12 908-920. MR1925445

[7] Bertoin, J. and Doney, R. A. (1994). On conditioning a random walk to stay nonnegative. Ann. Probab. 22 2152-2167. MR1331218

[8] Boman, J. and Lindskog, F. (2009). Support theorems for the Radon transform and CramérWold theorems. J. Theoret. Probab. 22 683-710.

[9] Buraczewski, D., Collamore, J. F., Damek, E. and Zienkiewicz, J. (2016). Large deviation estimates for exceedance times of perpetuity sequences and their dual processes. Ann. Probab. 44 3688-3739. MR3572322

[10] Buraczewski, D., Damek, E., GuivarC'H, Y. and Mentemeier, S. (2014). On multidimensional Mandelbrot cascades. J. Difference Equ. Appl. 20 1523-1567.

[11] Buraczewski, D., DameK, E. and Mikosch, T. (2016). Stochastic Models with Power Law Tails: The Equation $X=A X+B$. Springer, Berlin.

[12] Buraczewski, D., Damek, E. and Zienkiewicz, J. (2016). On the Kesten-Goldie constant. J. Difference Equ. Appl. 22 1646-1662. MR3590406

[13] Buraczewski, D. and Mentemeier, S. (2016). Precise large deviation results for products of random matrices. Ann. Inst. Henri Poincaré Probab. Stat. 52 1474-1513. MR3531716

[14] Collamore, J. F. (2009). Random recurrence equations and ruin in a Markov-dependent stochastic economic environment. Ann. Appl. Probab. 19 1404-1458. MR2538076 
[15] Collamore, J. F., Diao, G. and Vidyashankar, A. N. (2014). Rare event simulation for processes generated via stochastic fixed point equations. Ann. Appl. Probab. $242143-$ 2175.

[16] Collamore, J. F. and Mentemeier, S. (2016). Large excursions and conditioned laws for recursive sequences generated by random matrices. Preprint. Available at arXiv:1608.05175.

[17] Collamore, J. F. and Vidyashankar, A. N. (2013). Large deviation tail estimates and related limit laws for stochastic fixed point equations. In Random Matrices and Iterated Random Functions (G. Alsmeyer and M. Löwe, eds.) 91-117. Springer, Berlin.

[18] Collamore, J. F. and Vidyashankar, A. N. (2013). Tail estimates for stochastic fixed point equations via nonlinear renewal theory. Stochastic Process. Appl. 123 3378-3429.

[19] de HaAn, L., Resnick, S. I., Rootzén, H. and De VRies, C. G. (1989). Extremal behaviour of solutions to a stochastic difference equation with applications to $\mathrm{ARCH}$ processes. Stochastic Process. Appl. 32 213-224. MR1014450

[20] Dembo, A., Karlin, S. and Zeitouni, O. (1994). Large exceedances for multidimensional Lévy processes. Ann. Appl. Probab. 4 432-447. MR1272734

[21] EnriqueZ, N., SABot, C. and Zindy, O. (2009). A probabilistic representation of constants in Kesten's renewal theorem. Probab. Theory Related Fields 144 581-613.

[22] Feller, W. (1971). An Introduction to Probability Theory and Its Applications, Vol. II, 2nd ed. Wiley, New York.

[23] Goldie, C. M. (1991). Implicit renewal theory and tails of solutions of random equations. Ann. Appl. Probab. 1 126-166.

[24] Guivarc'H, Y. (1990). Sur une extension de la notion de loi semi-stable. Ann. Inst. Henri Poincaré Probab. Stat. 26 261-285.

[25] Guivarc'H, Y. and Le PAge, É. (2016). Spectral gap properties for linear random walks and Pareto's asymptotics for affine stochastic recursions. Ann. Inst. Henri Poincaré Probab. Stat. 52 503-574. MR3498000

[26] Hennion, H. (1997). Limit theorems for products of positive random matrices. Ann. Probab. $251545-1587$.

[27] Iglehart, D. L. (1972). Extreme values in the GI/G/1 queue. Ann. Math. Stat. 43 627-635.

[28] Kesten, H. (1973). Random difference equations and renewal theory for products of random matrices. Acta Math. 131 207-248. MR0440724

[29] Kesten, H. (1974). Renewal theory for functionals of a Markov chain with general state space. Ann. Probab. 2 355-386.

[30] Kesten, H., Kozlov, M. V. and Spitzer, F. (1975). A limit law for random walk in a random environment. Compos. Math. 30 145-168.

[31] Leadbetter, M. R. and Rootzén, H. (1988). Extremal theory for stochastic processes. Ann. Probab. 16 431-478.

[32] LETAC, G. (1986). A contraction principle for certain Markov chains and its applications. In Random Matrices and Their Applications (Brunswick, Maine, 1984). Contemp. Math. 50 263-273. Amer. Math. Soc., Providence, RI. MR0841098

[33] LiU, Q. (2000). On generalized multiplicative cascades. Stochastic Process. Appl. 86 263-286. MR1741808

[34] Melfi, V. F. (1992). Nonlinear Markov renewal theory with statistical applications. Ann. Probab. $20753-771$.

[35] Melfi, V. F. (1994). Nonlinear renewal theory for Markov random walks. Stochastic Process. Appl. 54 71-93. MR1302695

[36] Mentemeier, S. (2013). On Multivariate Stochastic Fixed Point Equations: The Smoothing Transform and Random Difference Equations. Ph.D. thesis, Westfälische WilhelmsUniversität Münster. 
[37] Meyn, S. and Tweedie, R. (1993). Markov Chains and Stochastic Stability. Springer, Berlin.

[38] Mikosch, T. (2003). Modeling dependence and tails of financial time series. In Extreme Values in Finance, Telecommunications, and the Environment (B. Finkenstädt and H. Rootzén, eds.) 185-286. Chapman \& Hall, Boca Raton, FL.

[39] MireK, M. (2011). Heavy tail phenomenon and convergence to stable laws for iterated Lipschitz maps. Probab. Theory Related Fields 151 705-734.

[40] Nummelin, E. (1984). General Irreducible Markov Chains and Non-negative Operators. Cambridge Univ. Press, Cambridge.

[41] Perfekt, R. (1994). Extremal behaviour of stationary Markov chains with applications. Ann. Appl. Probab. 4 529-548.

[42] Perfekt, R. (1997). Extreme value theory for a class of Markov chains with values in $\mathbb{R}^{d}$. Adv. in Appl. Probab. 29 138-164.

[43] Resnick, S. (2004). On the foundations of multivariate heavy-tail analysis. J. Appl. Probab. 41A 191-212.

[44] Roitershtein, A. (2007). One-dimensional linear recursions with Markov-dependent coefficients. Ann. Appl. Probab. 17 572-608. MR2308336

[45] Shurenkov, V. M. (1984). On Markov renewal theory. Theory Probab. Appl. 29 247-265.

[46] Siegmund, D. (1985). Sequential Analysis: Tests and Confidence Intervals. Springer, New York.

[47] Solomon, F. (1972). Random walks in random environment. Ph.D. dissertation, Cornell Univ., Ithaca, NY.

[48] Solomon, F. (1975). Random walks in random environment. Ann. Probab. 3 1-31.

[49] VAn Der VAART, A. W. and Wellner, J. A. (1996). Weak Convergence and Empirical Processes: With Applications to Statistics. Springer, New York. MR1385671

[50] WANG, H. M. (2013). A note on multitype branching process with bounded immigration in random environment. Acta Math. Sin. (Engl. Ser.) 29 1095-1110.

DEPARTMENT OF MATHEMATICAL SCIENCES

UNIVERSITY OF COPENHAGEN

UNIVERSITETSPARKEN 5

DK-2100 COPENHAGEN $\varnothing$

DENMARK

E-MAIL: collamore@math.ku.dk
FAKULTÄT FÜR MATHEMATIK, LEHRSTUHL IV TEChNISCHE UNIVERSITÄT DORTMUND VOGELPOTHSWEG 87

44227 DORTMUND

GERMANY

E-MAIL: sebastian.mentemeier@tu-dortmund.de 